University of Louisville

ThinkIR: The University of Louisville's Institutional Repository

Electronic Theses and Dissertations

8-2014

\title{
Semi-blind robust indentification and robust control approach to personalized anemia management.
}

\author{
Elom Akabua \\ University of Louisville
}

Follow this and additional works at: https://ir.library.louisville.edu/etd

Part of the Electrical and Computer Engineering Commons

\section{Recommended Citation}

Akabua, Elom, "Semi-blind robust indentification and robust control approach to personalized anemia management." (2014). Electronic Theses and Dissertations. Paper 20.

https://doi.org/10.18297/etd/20

This Doctoral Dissertation is brought to you for free and open access by ThinkIR: The University of Louisville's Institutional Repository. It has been accepted for inclusion in Electronic Theses and Dissertations by an authorized administrator of ThinkIR: The University of Louisville's Institutional Repository. This title appears here courtesy of the author, who has retained all other copyrights. For more information, please contact thinkir@louisville.edu. 


\title{
SEMI-BLIND ROBUST IDENTIFICATION AND ROBUST CONTROL APPROACH TO PERSONALIZED ANEMIA MANAGEMENT
}

\author{
By \\ Elom Akabua \\ M.S., University of Louisville, 2007 \\ A Dissertation \\ Submitted to the Faculty of the \\ J.B. Speed School of Engineering of the University of Louisville \\ in Partial Fulfillment of the Requirements \\ for the Degree of
}

Doctor of Philosophy

Department of Electrical and Computer Engineering

University of Louisville

Louisville, Kentucky

August 2014 



\title{
SEMI-BLIND ROBUST IDENTIFICATION AND ROBUST CONTROL APPROACH TO PERSONALIZED ANEMIA MANAGEMENT
}

\author{
Submitted by \\ Elom Akabua \\ M.S., University of Louisville, 2007
}

A Dissertation Approved on

10 June 2014

by the Following Reading and Examination Committee:

Tamer Inanc, Ph.D., Dissertation Director

Michael Brier, Ph.D.

Michael McIntyre, Ph.D.

Yongzhi Steve Xu, Ph.D.

Jacek Zurada, Ph.D. 


\section{DEDICATION}

Dedicated to my Father and Mother for their love and support. 


\section{ACKNOWLEDGMENTS}

My sincere appreciation to my thesis advisor, Dr. Tamer Inanc. His guidance and constant suggestions resulted in the final version of this work. He is not just great as my thesis director but also as a person with a high level of patience and understanding whose composure I can only hope to acquire someday. I would also like to thank Dr. Jacek Zurada for all the helpful suggestions and for agreeing to serve as a member of my dissertation committee. The weekly (and often biweekly) seminar he jointly organized with Dr. Inanc was informative and a confidence building for young researchers. I would like to thank the rest my committee members Dr. Michael Brier, Dr. Michael McIntyre, and Dr. Steve Xu for agreeing to serve on my committee. I am grateful to Dr. Adam Gaweda and Dr. Michael Brier for all the valuable discussions on patient modeling and for providing the needed data for this project. My appreciation goes to Ms. Lisa Bell for all her help.

More importantly, I would like to thank my parents Dr. Kojo Akabua and Mrs. Theresa Akabua for believing in my abilities and providing me the opportunity to apply myself. "aim for the stars....". Thank you dad for establishing the framework. I indeed "stand on the shoulders of a giant". To all my siblings: Juliana, Lilian, Ewoenam, Delali, Mawunyo, and Eyram, it is of a great honor to be your brother. And to the newly added members to the family: my nephew Joshua and niece Sydelle, welcome to the world.

Over the years, I have had the opportunity to meet some great people whose influence have greatly impacted both my personal and professional life. I would like to use this opportunity to thank some of these individuals-some of whose names have 
eluded me but certainly not forgotten. I would like to thank the following individuals for making this journey a joyous one: David Nfodjo, Kwesi Justice Kwarteng, Patrick Mono, Rami Mahdi, Mohammed Alsamna, Loay Elbasyouni, and Wiseman Koster. I would also like to thank all my former labmates from the ARCS lab for all the valuable discussions: Weizhong Zhang, Sara Shafaei, and Yinan Cui. 


\begin{abstract}
SEMI-BLIND ROBUST IDENTIFICATION AND ROBUST CONTROL APPROACH TO PERSONALIZED ANEMIA MANAGEMENT

Elom Akabua
\end{abstract}

2014-06-10

The homeostatic blood hemoglobin $(\mathrm{Hb})$ content of a healthy individual varies between the range of $14-18 \mathrm{~g} / \mathrm{dL}$ for a male and $12-16 \mathrm{~g} / \mathrm{dL}$ for a female. This quantity provides an estimate of red blood cell (RBC) count in circulation at any given moment. RBC is a protein carrying substance that transports oxygen from the lungs to other tissues in the body and is synthesized by the kidney through a process known as erythropoiesis where erythropoietin is secreted in response to hypoxia. In this regard, the kidneys act not only as a controller but also as a sensor in regulating RBC levels. Patients with chronic kidney diseases (CKD) have dysfunctional kidneys that compromise these fundamental kidney functions. Consequently, anemia is developed.

Anemics of $\mathrm{CKD}$ have low levels of $\mathrm{Hb}$ that must be controlled and properly regulated to the appropriate therapeutic range. Until the discovery of recombinant human erythropoietin (EPO) over three decades ago, treatment procedure of anemia conditions primarily involved repeated blood transfusions-a process known to be associated with several other health related complications. This discovery resulted in a paradigm shift in anemia management from blood transfusions to dosage therapies. The main objective of anemia management with EPO is to increase patients' hemoglobin level from low to a suitable therapeutic range as defined by the National Kidney Foundation-Kidney Dis- 
ease Outcomes Quality Initiative (NKF-KDOI) to be in the range of 10 - $12 \mathrm{~g} / \mathrm{dL}$ while avoiding response values beyond $14 \mathrm{~g} / \mathrm{dL}$ to prevent other complications associated with EPO medication. It is therefore imperative that clinicians balance dosage efficacy and toxicity in anemia management therapies. At most treatment facilities, protocols are developed to conform to NKF-KDOI recommendations. These protocols are generally based on EPO packet inserts and the expected $\mathrm{Hb}$ responses from the average patient. The inevitable variability within the patient group makes this "one-size-fits-all" dosing scheme non-optimal, at best, and potentially dangerous for certain group of patients that do not adhere to the notion of expected "average" response. A dosing strategy that is tailored to the individual patients' response to EPO medication could provide a better alternative to the current treatment methods.

An objective of this work is to develop EPO dosing strategies tailored to the individual patients using robust identification techniques and modern feedback control methods. First, a unique model is developed based on $\mathrm{Hb}$ responses and dosage EPO of the individual patients using semi-blind robust identification techniques. This provides a nominal model and a quantitative information on model uncertainty that accounts for other possible patient's dynamics not considered in the modeling process. This is in the framework of generalized interpolation theory. Then, from the derived nominal model and the associated uncertainty information, robust controller is designed via the $\mu / \mathrm{H}_{\infty}$ synthesis methods to provide a new dosing strategies for the individual patients. The $\mathrm{H}_{\infty}$ control theory has a feature of minimizing the influence of some unknown worstcase gain disturbance on a system. Finally, a framework is provided to strategize dosing protocols for newly admitted patients. 


\section{TABLE OF CONTENTS}

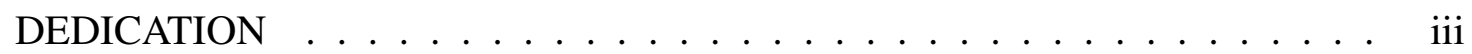

ACKNOWLEDGMENTS ............................. iv

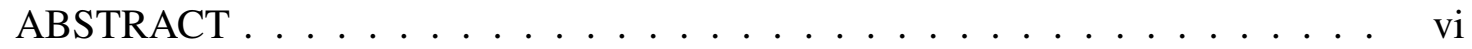

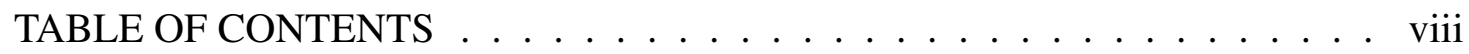

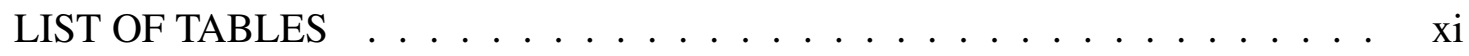

LIST OF FIGURES . . . . . . . . . . . . . . . . . . . . xii

CHAPTER

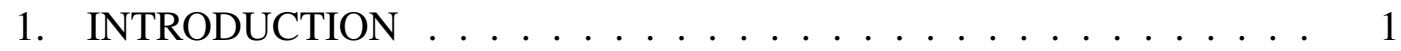

1.1 Nonparametric Identification Techniques in Dosage

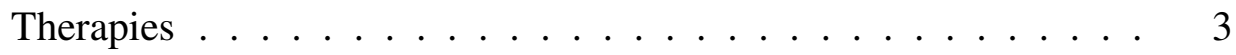

1.2 Model Validation . . . . . . . . . . . . . . 6

2. PRELIMINARIES ........................ 7

2.1 Notations ..................... 7

2.2 Signals and Systems $\ldots \ldots \ldots$. . . . . . . . . . . . 9

2.3 Linear Matrix Inequalities . . . . . . . . . . . . . . 11

3. ANEMIA MANAGEMENT PROBLEM . . . . . . . . . . . . . 13

3.1 Introduction . . . . . . . . . . . . . . 13

3.2 Definition ........................... 14

3.3 Erythropoiesis Drug Delivery Strategy . . . . . . . . . . . 15

3.4 Anemia Management as a Feedback Control Problem . . . . . . . . 16

4. SEMI-BLIND ROBUST IDENTIFICATION . . . . . . . . . . . . . . 18 
4.1 Robust Identification: An Overview . . . . . . . . . . . . . . . 18

4.2 Nonparametric Identification in Physiological Systems . . . . . . . 20

4.3 Robust Identification in Time Domain: An Overview . . . . . . . . 22

4.4 Nonparametric $\ell_{1}$ Robust Identification from Time Domain Data . . 24

4.4.1 The Robust Identification Problem . . . . . . . . . . 27

4.4 .2 Parametric Inclusion . . . . . . . . . . . 31

4.5 Semi-Blind Robust Identification of LTI Systems . . . . . . . . . 34

5. SEMI-BLIND ROBUST IDENTIFICATION APPROACH TO ANEMIA

MANAGEMENT PROBLEM . . . . . . . . . . . . . . . . . . 37

5.1 Individualizing Anemia Patients . . . . . . . . . . . . . 37

5.1.1 Performance Measure .............. . 42

5.2 Worst-Case Prediction Error Bounds . . . . . . . . . . . . . 50

5.3 ARX Model . . . . . . . . . . . . . . . . . 51

6. ROBUST CONTROL SYNTHESIS FOR ANEMIA MANAGEMENT

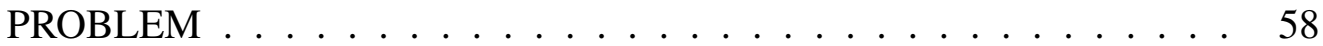

6.1 Robust Control synthesis . . . . . . . . . . . . 58

6.2 Robust Controller Design: Some Definitions _ . . . . . . . . . . . 60

6.2 .1 Nominal Stability (NS) . . . . . . . . . . 60

6.2 .2 Nominal Performance (NP) . . . . . . . . . . . 60

6.2 .3 Robust Stability $(\mathrm{RS}) \ldots \ldots$. . . . . . . . 61

6.2.4 Robust Performance (RP) . . . . . . . . . . . . . 61

6.3 Robust Control Design Solution to the Anemia Management Problem 62

6.3.1 Generalized Plant Description . . . . . . . . . 63

6.4 Weighting Functions ................. 64

6.5 Augmented Plant . . . . . . . . . . . . . . . . . 64

$6.6 \mathrm{H}_{\infty}$-Control Synthesis and Results . . . . . . . . . . . . . . . 66

6.7 Robust Control Design for the Initial Dose Regimen . . . . . . . . 69 
6.7 .1 Baseline Hb Level . . . . . . . . . . . . . . . 69

6.7.2 Weighting Function . . . . . . . . . . 70

6.8 Controller Design . . . . . . . . . . . . . . . 71

6.8 .1 Results ................... 71

6.9 Parameter Space Partition and Results . . . . . . . . . . . . . 72

7. CONCLUSIONS AND FUTURE WORK . . . . . . . . . . . . 78

7.1 Conclusion ..................... 78

7.2 Directions for Future Research . . . . . . . . . . . . . . . . . 79

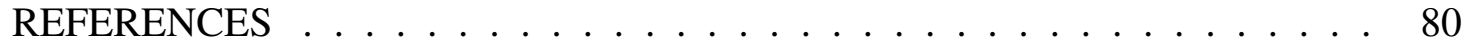

CURRICULUM VITAE . . . . . . . . . . . . . . . . 87 


\section{LIST OF TABLES}

5.1 Some a priori parameter information for the selected patients of poor responders patients \#1, \#28, and \#30 and normal responders, patients \#6 and \#37. $N$ represents the number of data points used in the identification and we indicated with a check mark whether patient belongs to a responder or non-responder groups. . . . . . . 40

5.2 Average and maximum prediction error for the selected patients . 43

5.3 Average and maximum prediction errors from ARX results. . . . . 53

6.1 Low, Mid, and High values of $k$ and $\tau$ parameters. . . . . . . . 70

6.2 Partitioned $k$ and $\tau$ parameter ranges. . . . . . . . . . 74 


\section{LIST OF FIGURES}

1.1 Patient-Physician interaction in a feedback loop. . . . . . . . 2

1.2 Blind identification with unknown system $h(\cdot)$ and input $u(\cdot) \ldots . \quad 5$

$2.1 \quad$ Signal representation. . . . . . . . . . . . . 10

3.1 Simulated $\mathrm{Hb}$ concentration of the desired (left) vs actual (right) observations. .......................... 14

4.1 Bias-variance tradeoff in model complexity level. . . . . . . . . 21

4.2 An LTI block diagram with input $\mathbf{u}$ and measured output $\mathbf{y}$. Noise signal $\eta$ is unknown but bounded while $\mathrm{z}$ is the unmeasurable signal coming from the system. . . . . . . . . . . . 24

4.3 Response plot with a priori and a posteriori information. . . . . 26

4.4 Representation of a system described by a nominal model $G_{0}$ and an additive uncertainty bound $\Delta . \ldots \ldots 26$

$4.5 \quad$ An overview of the robust identification technique. . . . . . . 29

5.1 Anemia Patient model connected with semi-blind robust identification configuration .................... 39

5.2 This figure represents the parametric portion of the system. . . . . 39 
5.3 Semi-blind robust identification results for patient \#1. [Top] $\mathrm{Hb}$ prediction with a vertical line used to demarcate data used for identification and for forecasting. A (+) is used to denote experimental data used during the identification and (*) is used to denote data not used in the identification. (o) symbol is used to indicate predicted results with $3^{r d}$ order model. [Bottom] Administered erythropoietin dose in x1000 Units per week. . . . . . . . . . . 44

5.4 Semi-blind robust identification results for patient \#28. [Top] $\mathrm{Hb}$ prediction with a vertical line used to demarcate data used for identification and for forecasting. A (+) is used to denote experimental data used during the identification and (*) is used to denote data not used in the identification. (o) symbol is used to indicate predicted results with $2^{\text {nd }}$ order model. [Bottom] Administered erythropoietin dose in x1000 Units per week. . . . . . . . . . 45

5.5 Semi-blind robust identification results for patient \#30. [Top] $\mathrm{Hb}$ prediction with a vertical line used to demarcate data used for identification and for forecasting. A (+) is used to denote experimental data used during the identification and (*) is used to denote data not used in the identification. (o) symbol is used to indicate predicted results with $1^{\text {st }}$ order model. [Bottom] Administered erythropoietin dose in $\mathrm{x} 1000$ Units per week. . . . . . . . . . 46 
5.6 Semi-blind robust identification results for patient \#6. [Top] $\mathrm{Hb}$ prediction with a vertical line used to demarcate data used for identification and for forecasting. A (+) is used to denote experimental data used during the identification and (*) is used to denote data not used in the identification. (o) symbol is used to indicate predicted results with $2^{\text {nd }}$ order model. [Bottom] Administered erythropoietin dose in $\mathrm{x} 1000$ Units per week. . . . . . . . . . . . 47

5.7 Semi-blind robust identification results for patient \#37. [Top] $\mathrm{Hb}$ prediction with a vertical line used to demarcate data used for identification and for forecasting. A (+) is used to denote experimental data used during the identification and $(*)$ is used to denote data not used in the identification. (o) symbol is used to indicate predicted results with $1^{\text {st }}$ order model. [Bottom] Administered erythropoietin dose in x1000 Units per week. . . . . . . . . . . 48

5.8 Maximum prediction error for all patients from the semi-blind identification technique. . . . . . . . . . . . ARX model results for patient \#37. [Top] $\mathrm{Hb}$ prediction with a vertical line used to demarcate data used for identification and for forecasting. A (+) is used to denote experimental data used during the identification and (*) is used to denote data not used in the identification. (o) symbol is used to indicate predicted results with $1^{\text {st }}$ order model. [Bottom] Administered erythropoietin dose in x1000 Units per week. . . . . . . . . . . . . . 53 
ARX model results for patient \#6. [Top] Hb prediction with a vertical line used to demarcate data used for identification and for forecasting. A (+) is used to denote experimental data used during the identification and $(*)$ is used to denote data not used in the identification. (o) symbol is used to indicate predicted results with $2^{\text {nd }}$ order model. [Bottom] Administered erythropoietin dose in x1000 Units per week. . . . . . . . . . . . . . 54

5.11 ARX model results for patient \#30. [Top] $\mathrm{Hb}$ prediction with a vertical line used to demarcate data used for identification and for forecasting. A (+) is used to denote experimental data used during the identification and $(*)$ is used to denote data not used in the identification. (o) symbol is used to indicate predicted results with $1^{\text {st }}$ order model. [Bottom] Administered erythropoietin dose in x1000 Units per week. . . . . . . . . . . . . 55

5.12 ARX model results for patient \#28. [Top] Hb prediction with a vertical line used to demarcate data used for identification and for forecasting. A (+) is used to denote experimental data used during the identification and $(*)$ is used to denote data not used in the identification. (o) symbol is used to indicate predicted results with $2^{\text {nd }}$ order model. [Bottom] Administered erythropoietin dose in x1000 Units per week. . . . . . . . . . . . . 56 
5.13 ARX model results for patient \#1. [Top] Hb prediction with a vertical line used to demarcate data used for identification and for forecasting. A (+) is used to denote experimental data used during the identification and $(*)$ is used to denote data not used in the identification. (o) symbol is used to indicate predicted results with $3^{\text {rd }}$ order model. [Bottom] Administered erythropoietin dose in x1000 Units per week. . . . . . . . . . . . . 57

6.1 Feedback system. . . . . . . . . . . . . 59

6.2 Multiplicative uncertainty in a feedback loop. . . . . . . . . . 60

$6.3 \quad$ Generalized Plant Framework . . . . . . . . . . . . 63

6.4 Anemia management as a robust control problem in a feedback loop. 65

$6.5 \quad$ Uncertainty feedback system loop. . . . . . . . . . 65

6.6 Robust control results for patient \#1. [Top] Hemoglobin response.

[Bottom:] EPO values. . . . . . . . . . . . . 67

6.7 Robust control results for patient \#37. [Top] Hemoglobin response.

[Bottom:] EPO values. . . . . . . . . . . . . 68

$6.8 \quad$ Step response of partitioned system plant . . . . . . . . . 70

$6.9 \quad$ Bode plot of uncertainty function. . . . . . . . . 71

6.10 Step response of possible plant dynamics. . . . . . . . . 72

6.11 Controller result for the general transfer function equation. Top:

$\mathrm{Hb}$ response stabilized at $11 \mathrm{~g} / \mathrm{dL}$. Bottom: EPO medication. . . . 73

6.12 $\mathrm{Hb}($ top) vs. EPO (bottom) for poorly responsive patients . . . . 75

$6.13 \quad \mathrm{Hb}$ (top) vs. EPO (bottom) for average responsive patients . . . 76

$6.14 \quad \mathrm{Hb}$ (top) vs. EPO (bottom) for good responsive patients . . . . . 77 


\section{CHAPTER 1 INTRODUCTION}

Anemia of End-Stage Renal Disease (ESRD) results from insufficient production of erythropoietin, a glycoprotein promoting the growth of red blood cells (RBC) in the bone marrow as a response to hypoxia. When RBC level is low, the kidneys secrete erythropoietin to stimulate the proliferation of red blood cell precursors that eventually lead to an increased level of matured RBC counts. RBC contains hemoglobin $(\mathrm{Hb})$ that transports oxygen from the lungs to other tissues. Patients of anemia due to ESRD have dysfunctional kidneys that are incapable of producing sufficient endogenous erythropoietin to initiate the RBC production process to sustain a good quality of life. As a result, they develop anemia.

Until a little over three decades ago, management of anemia conditions primarily involved repeated blood transfusion, a procedure known to be associated to several other health related complications. However, the discovery of recombinant human erythropoietin (EPO) over three decades ago [1] shifted anemia treatment process from blood transfusion to dosage therapy. The overall objective is to elevate the low $\mathrm{Hb}$ level to an effective therapeutic $\mathrm{Hb}$ range. Although the dosing of EPO has been shown to interrupt the progress of anemia of ESRD and in some cases corrects it [2-4], the issues of developing comprehensive dosing strategies that consistently meet the design objectives remains a major challenge. This is primarily due to the large patient variability in erythropoietic responses. As a consequence, the decision process involved in EPO dosage medication consists mostly of trial-and-error [5]. To standardize anemia 


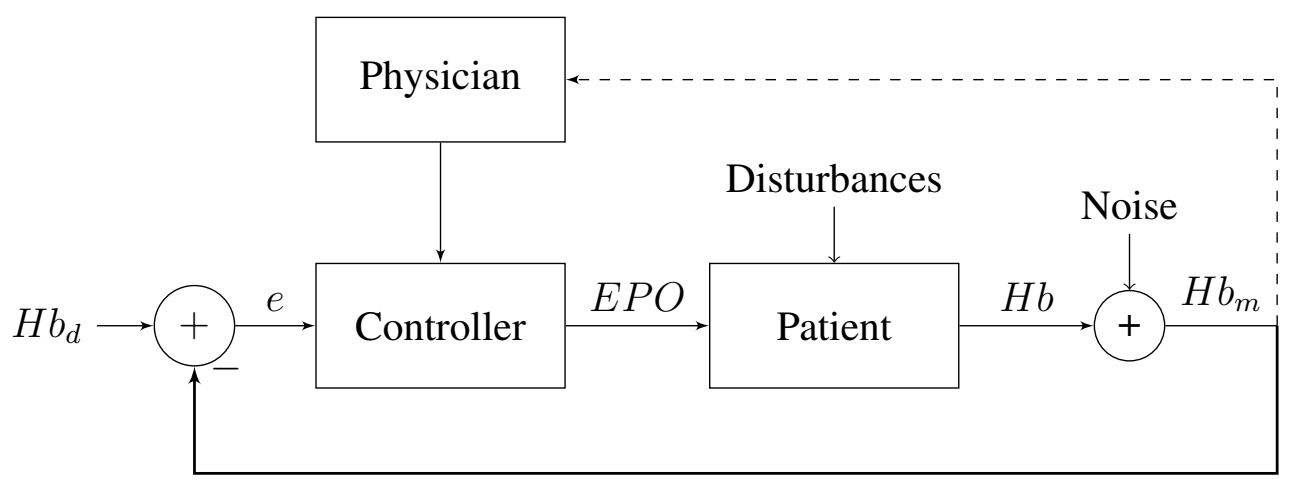

FIGURE 1.1: Patient-Physician interaction in a feedback loop.

treatment process, the National Kidney Foundation's Kidney Disease Outcomes Quality Initiative (NKF-KDOQI) has established guidelines with the recommendation that the hemoglobin levels of patients of ESRD to be maintained within a narrow range of 10-12 $\mathrm{g} / \mathrm{dL}[6]$.

Many therapeutic solutions are closely related to system control problems and feedback measurements [7-9]. Regarding EPO dosage therapy, this relation is summarized in Figure 1.1. In this figure, $\mathrm{Hb}_{d}$ and $\mathrm{Hb}_{m}$ represent certain desired and measured hemoglobin levels, respectively. The goal of a physician is to gather information on the patient, including past input and output measurement data, and apply the proper dose of EPO in an attempt to regulate the subsequent $\mathrm{Hb}$ values to some desired range. However, factors such as noise and disturbance in the feedback configuration make this objective challenging. This is a typical phenomenon in most practical drug dosage treatment methods. The aim is to translate the anemia management problem into a feedback control problem and use the tools of modern feedback control techniques to develop a dosing strategy that is consistent and robust to unknown disturbances. Achieving this objective requires unique mathematical model for each patient that describes the relationship between measured hemoglobin level and input erythropoietin. 


\subsection{Nonparametric IdEnTIFICATION TECHNiQUes In Dosage THERAPIES}

A necessary requirement for feedback control design methods is a mathematical description of the process to be controlled. For certain systems such as mechanical and electromechanical systems, this description can be estimated based on first principles. Modeling from first principles approach is not suitable for certain systems with complex dynamics. Using classical system identification methods requires sufficiently large sample size, a luxury that is not common in modeling with clinical data with low sampling frequency $[10,11]$. In addition, the classical system identification techniques require that model structure to be specified in advance, a requirement that is often done in trial-and-error fashion.

The classical system identification technique works well, provided the specified model structure is in agreement with that of the true system structure. By contrast, if the assumed structure differs from that of the system's, results from the technique may produce an unsatisfactory outcome $[12,13]$. This is primarily due to the fact that this identification method only yields a single mathematical model that is presumed to be capable of describing the overall dynamics of the actual system, regardless of its complexity. System dynamics and parameter values may change and neglecting such eventualities at the modeling stage may yield inferior results. Additionally, the very notion of randomness in measurement values may be questionable. It is well understood that any derived mathematical description of a system is a simplified version of the true plant and that it is impossible to provide models that completely describe the true behavior of a system. Changes in estimated parameter values during operation and unknown uncertainty entering the process must be considered at the modeling stage. This change in parameter values during operation leads to the concept of plant uncertainty. Another source of uncertainty is the one due to external signals including measured and unmea- 
sured disturbances, referenced input signal, etc. To completely describe the dynamic behavior of any system, it is essential to consider the effect of uncertainty on the overall behavior of the model.

Basic requirements for robust control design technique are a nominal model and a quantifiable information on the associated uncertainty in the derived model. As a result, any robust control oriented identification procedure must deliver not only such nominal model but also a quantitative information on the model uncertainty. These requirements for robust control initiated a new identification methods termed controloriented identification in the 90s (see [14-19]). The techniques of robust identification diverge considerably from that of classical system identification methods. Particularly, the procedure does not require any statistical information on noise affecting measurements nor does it require information on the structure of the system to be identified. In this identification procedure, the only a priori requirement is that the following three constants must be available: the maximum gain of the system $K$, the stability margin of the system response $\rho$, and a bound on noise $\epsilon$. Depending on the nature of the a posteriori information, the robust identification techniques may lead to different identification methods.

When experimental data originates from frequency domain ${ }^{1}$, the technique leads to $H_{\infty}$-identification procedure (see [14-16,20-23]) which measures the uncertainty bounds in terms of the $H_{\infty}$-norm and is sufficient for $H_{\infty}$ optimal controller or $\mu$ synthesis design techniques. On the other hand, when experimental data originate from time domain data, the $\ell_{1}$-identification technique is used ( see [24-28]) which provides an $\ell_{1}$ error bounds and is suitable for $\ell_{1}$ optimal control design techniques [29]. Occasionally, both time and frequency domain data may be available or generated from a single plant. In this case, the mixed $H_{\infty} / \ell_{1}$ robust identification procedure is used

\footnotetext{
${ }^{1}$ Frequency domain data are data obtained from frequency response of the system while time domain data are those obtained from time response of the system
} 


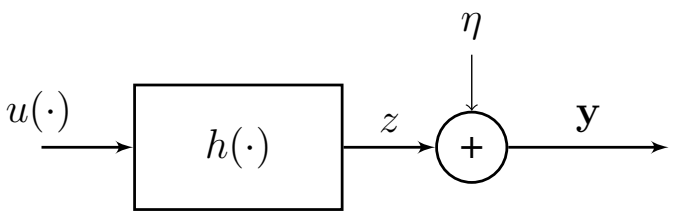

FIGURE 1.2-Blind identification with unknown system $h(\cdot)$ and input $u(\cdot)$.

(see [30-33]). Obviously, with both time and frequency domain experimental data available, a much smaller consistency set can be derived; however, for most systems, it is generally a challenge or costly to perform frequency domain experiment. Since the clinical data available for this work is of the time domain nature, this work focuses on the $\ell_{1}$ identification technique.

In modeling from measurement data, a common assumption is that experimental data are generated precisely at time $t_{0}=0$ while ignoring the effects of the system dynamics prior to the identification step. While this assumption simplifies the problem, it may introduce a very high prediction error in the model. To address this problem, a new approach to identification with nonzero initial conditions is considered. This is achieved by a new identification method called semi-blind robust identification [34]. In this recently developed identification method, the goal is to estimate, in addition to the system's impulse responses, the system dynamics prior to the identification process using only output measurements. Figure 1.2 shows the objectives of the technique. From the figure, both the system and the initial inputs are unknown and are to be determined from the measurement output. This problem generally leads to a non-convex optimization problem. However, by relaxing the problem by assuming a minimal information on the system, it is shown that the problem can be solved [35]. 


\subsection{Model VAlidation}

Models obtained from the robust identification technique must be validated. If a derived model is combined with the associated uncertainty bound is found not to be able to produce the system experimental data, a new a priori information on the system must be assumed or an additional experiment must be constructed. It should be emphasized here that one cannot truly validate a model but rather invalidate it since it is always possible to provide conditions to falsify any given model. To this effect, when outputs of the estimated model is combined with the corresponding uncertainty bound and is found to be consistent with the system output measurements, it is said that the estimated model is not invalidated. On the other hands, when the outputs measurements of the estimated model and its uncertainty bounds does not correspond with the system output measurement, it is said that the estimated model is invalidated. The problems of model (in)validation in linear time invariant (LTI) systems is not new. This

problem was initiated by Smith etc. [36] and has since attracted the attention of several researchers [37-39]. Techniques exist to partially solve this problem for unstructured uncertainty models. The objective here is to reduced the problem into a convex optimization problem that can be solved with any optimization tools. For structured LTI uncertainty models, this problem remains to be an NP-hard problem [40]. It should be mentioned that even in the case of unstructured uncertainty model (in)validation problems solved above, the assumption is that the initial conditions of the system model are zero. 


\section{CHAPTER 2}

\section{PRELIMINARIES}

This chapter provides an overview of some needed notations, equations, functions, and generalized interpolation conditions that are used in the thesis.

\subsection{Notations}

Below summary is a list of notations used throughout the thesis.

$\mathbb{R}$ set of real numbers

$\mathbb{C}$ set of complex numbers

$\mathbb{Z}\left(\mathbb{Z}_{+}\right)$ set of integers (non-negative)

$\mathrm{x}$ vector containing real values (otherwise stated)

$\mathbf{x}_{k}$ $k^{\text {th }}$ element of vector $\mathbf{x}$

$\|x\|_{p}$ p-norm of vector $\mathbf{x}$ defined as $\|x\|_{p} \doteq\left(\sum_{k=1}^{m}\left|x_{k}\right|^{p}\right)^{\frac{1}{p}}$, where $p \in$ $[1, \infty),\|x\|_{\infty} \doteq \max _{k=1, \ldots m}\left|x_{k}\right|$

$\|A\|_{p}$ induced p-norm of matrix A, such that $\|A\|_{p} \doteq \max \frac{\|A x\|_{q}}{\|x\|_{q}}$ for $x \neq 0$ conjugate transpose of matrix $\mathbf{A}$ eigenvalue of matrix $\mathbf{A}$ maximum singular value of matrix $\mathbf{A}$ 

$\mathbf{A}>(\geq) \mathbf{0}$
$\mathbf{A}=\mathbf{A}^{T}$ positive (semi) definite of matrix $\mathbf{A}$ such that $\mathbf{x}^{\mathbf{T}} \mathbf{A} \mathbf{x}>(\geq$
) $0 \forall \mathbf{x} \in \mathbb{C}^{n}, \mathbf{x} \neq \mathbf{0}$
$\mathcal{B} \mathcal{X}(\gamma)$
an open $\gamma$-ball in $\mathcal{X}$ such that $\mathcal{B X}(\gamma)=\left\{x \in \mathcal{X}:\|x\|_{\mathcal{X}}<\gamma\right\}$
$\overline{\mathcal{B X}}(\gamma)$
closure of $\mathcal{B} \mathcal{X}(\gamma)$
$\mathcal{B X}(\overline{\mathcal{B X}})$
open (closed) unit ball in $\mathcal{X}$
$(\mathcal{X}, m)$
metric space of elements in $\mathcal{X}$ equipped with the metric $m\left(x_{1}, x_{2}\right)$
$r(\mathcal{A})$
radius of a set $\mathcal{A} \subseteq \mathcal{X}: r(\mathcal{A}) \doteq \inf _{x \in \mathcal{X}} \sup _{a \in \mathcal{A}} m(x, a)$
$d(\mathcal{A})$
diameter of a set $\mathcal{A} \subseteq \mathcal{X}: d(\mathcal{A}) \doteq \sup _{x, a \in \mathcal{A}} m(x, a)$
$\ell_{p}^{m}$
extended Banach space of vector valued real sequences equipped with norm: $\|x\|_{p}$
$\mathcal{L}_{\infty}$
Lebesgue space of complex-valued matrix functions essentially bounded on the unit circle equipped with the norm: $\|G\|_{\infty} \doteq$ ess $\sup _{|z|=1} \bar{\sigma}(G(z))$
$\mathcal{H}_{\infty}$
subspace of functions in $\mathcal{L}_{\infty}$ with bounded analytic continuation inside the unit disk and equipped with the norm: $\|G\|_{\infty} \doteq$ ess $\sup _{|z|<1} \bar{\sigma}(G(z))$
$\mathcal{H}_{\infty, \rho}$ space of transfer matrices analytic in $|z| \leq \rho$ with the norm $\|G\|_{\infty, \rho} \bar{\sigma}(G(z))$
$\mathcal{X}(z) \quad$ right-sided Z-transform of real sequence $\{x\}: \mathcal{X}(z)=\sum_{i=0}^{\infty} x_{i} z^{i}$
$\mathbf{T}_{x}^{N} \quad$ lower triangular block Toeplitz matrix of a finite sequence $x_{k}, k=$ $0,1, \ldots, N-1$ defined as:
$\left[\begin{array}{cccc}x_{0} & 0 & \cdots & 0 \\ x_{1} & x_{0} & \cdots & 0 \\ \vdots & \ddots & \ddots & 0 \\ x_{N-1} & \cdots & x_{1} & x_{0}\end{array}\right]$ 

defined as:

$$
\left[\begin{array}{cccc}
x_{1} & x_{2} & \cdots & x_{N} \\
x_{2} & x_{3} & \cdots & x_{N+1} \\
\vdots & \ddots & \ddots & \vdots \\
x_{N} & x_{N+1} & \cdots & x_{2 N-1}
\end{array}\right]
$$

\subsection{SignALS AND SYSTEMS}

A signal is a time or space property of a physical phenomenon that can be used to convey information. Signals can be classified as having either a discrete or a continuous properties or both. Most natural signals are continuous; however, they can be discretized for analysis as seen in Figure 2.1 where a sine signal is discretized at an equally spaced time intervals to produce discrete samples.

A system is defined as a function that maps an input signal to a corresponding output signal. That is, given an input-output data sequence, an operator $G$ maps the input sequence to the output values. This function can be represented as either a (rational) complex valued function as defined in Equation (2.1) or as a state space representation of Equation (2.2).

$$
\begin{gathered}
G(z) \doteq \sum_{i=0}^{\infty} g_{i} z^{i} \\
x_{k+1}=A x_{k}+B u_{k} \\
y_{k}=C x_{k}+D u_{k}
\end{gathered}
$$




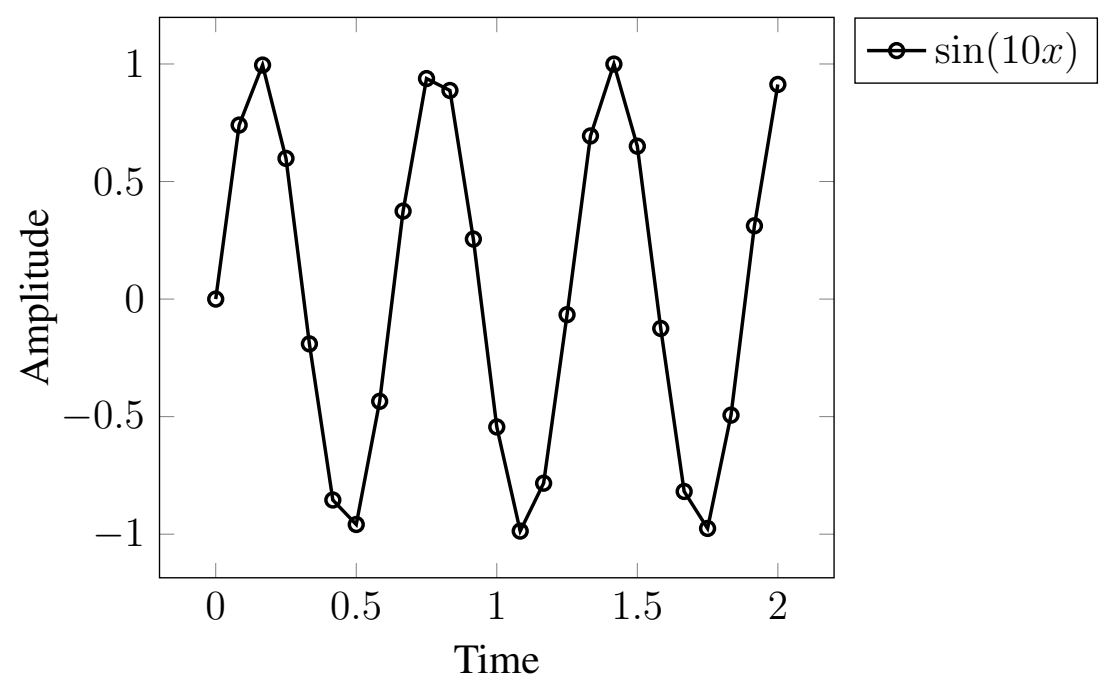

FIGURE 2.1 - Signal representation.

In Equation (2.1), $g_{i}$ represents the impulse response vector corresponding to system $G$. Similarly, parameters of Equation (2.2) are defined as follow: $x_{k} \in \mathbb{R}^{n}$ is the state of the system, $u_{k} \in \mathbb{R}^{p}$ defines the input sequence, and $y_{k} \in \mathbb{R}^{q}$ defines the output of the system. Parameters $A \in \mathcal{R}^{n \times n}, B \in \mathcal{R}^{n \times p}, C \in \mathcal{R}^{q \times n}$, and $D \in \mathcal{R}^{q \times p}$ represent state, input, output, and feedforward matrices of the system, respectively. A more compact form of representing the state space of Equation (2.2) is the minimal state-space realization form as shown in Equation (2.3) where the matrices carry the same meaning as indicated above.

$$
G \equiv\left(\begin{array}{c|c}
A & B \\
\hline C & D
\end{array}\right)
$$

Given the input sequence $u_{k}$ with a known system impulse response sequence, the corresponding output can be determined through the convolution sum as shown in Equation (2.4).

$$
y_{k}=G(z) u_{k}
$$




\subsection{LINEAR MATRIX INEQUALITIES}

Many control engineering problems can be translated into a set of Linear Matrix Inequalities (LMI) formulation and solved with any linear optimization solvers [41]. LMI techniques have been used in many application areas such as system estimation, identification, optimal design methods, etc. The technique benefits from its ability to combine various constraints and/or objectives problems into a numerically tractable one. Equation (2.5) shows the form of LMI constraints.

$$
L(x)=L_{0}+x_{1} L_{1}+x_{2} L_{2}+\cdots+x_{N} L_{N}<0
$$

where

- $x=\left(x_{1}, x_{2}, \ldots, x_{N}\right)$ is a vector of unknown scalar which is also the decision or the design variable

- $L_{0}, L_{1}, \ldots, L_{N}$ are some given symmetric matrices,

- " $<0$ " implies "negative definiteness", which is the largest eigenvalue of $L(x)$.

Constraints of the forms $L(x)>0$ and $L(x)<N(x)$ are special case of Equation (2.5) since they can be rewritten as $-L(x)<0$ and $L(x)-N(x)<0$, respectively. Indeed, several LMI constraint problems can be combined to form a single LMI problem as shown in Equation (2.7).

$$
\begin{gathered}
L_{0}(x)<0, L_{1}(x)<0, L_{2}(x)<0, \ldots, L_{N}(x)<0 \\
L(x)=\left[\begin{array}{cccc}
L_{0}(x) & 0 & \ldots & 0 \\
0 & L_{1}(x) & \ldots & 0 \\
\vdots & \ddots & \ddots & 0 \\
0 & \ldots & 0 & L_{N}(x)
\end{array}\right]<0
\end{gathered}
$$


LMI techniques can be extended to nonlinear control problems through Schur's complements transformation as summarized in Equation (2.8) [41].

$$
\begin{array}{r}
{\left[\begin{array}{rr}
Q(x) & S(x) \\
S(x)^{T} & R(x)
\end{array}\right]<0} \\
Q(x)=Q(x)^{T} \\
R(x)=R(x)^{T}
\end{array}
$$

Here, $S(x)$ depends affinely on " $x$ " and is equivalent to

$$
\begin{array}{r}
R(x)<0 \\
Q(x)-S(x) R(x)^{-1} S(x)^{T}<0
\end{array}
$$

Linear Matrix Inequalities problems are classified into three main categories [41]:

- Feasibility problem: This LMI types involves finding a solution $P$ to the LMI system $L(P)<0$

- Linear Objective Minimization problem: Minimize a convex objective under LMI constraints

$$
\begin{gathered}
\\
\min C^{P} \\
\text { s.t. } \quad L(P)<0
\end{gathered}
$$

- Generalized eigenvalue minimization problem: given by

$$
\text { s.t. } \quad\left\{\begin{array}{l}
\min \lambda \\
L(P)<\lambda B(P) \\
B(P)>0 \\
C(P)<0
\end{array}\right.
$$




\section{CHAPTER 3}

\section{ANEMIA MANAGEMENT PROBLEM}

In this chapter, an overview of the anemia management problem is reviewed. First, the anemia management "problem" is defined, then a review of some of the existing approaches in the literature that have been used to address this problem. Finally, anemia management as a feedback control problem is provided to conclude the chapter.

\subsection{INTRODUCTION}

A healthy individual synthesizes about $10^{10}$ red blood cells (RBC) per hour to maintain acceptable $\mathrm{Hb}$ level in the range of 12 to $18 \mathrm{~g} / \mathrm{dL}$ [42]. This process is driven by erythropoietin, a hormone produced primarily in the kidney cortex [2]. In chronic kidney disease (CKD) patients, natural production of this hormone is compromised leading to chronic anemia. The preferred treatment method of this condition involved external administration of EPO; however, determining the exact amount of the medication to administer to achieve treatment objectives while avoiding toxic side effects remains a major challenge. As a result, most EPO dosing strategies focus on treatment procedures based on "average" population-wide patient responses and EPO packet information. However, inter-patient and intra-individual variability in erythropoietic responses makes this dosing strategy non-optimal to an already costly medication and potentially dangerous aberrant patient groups. 

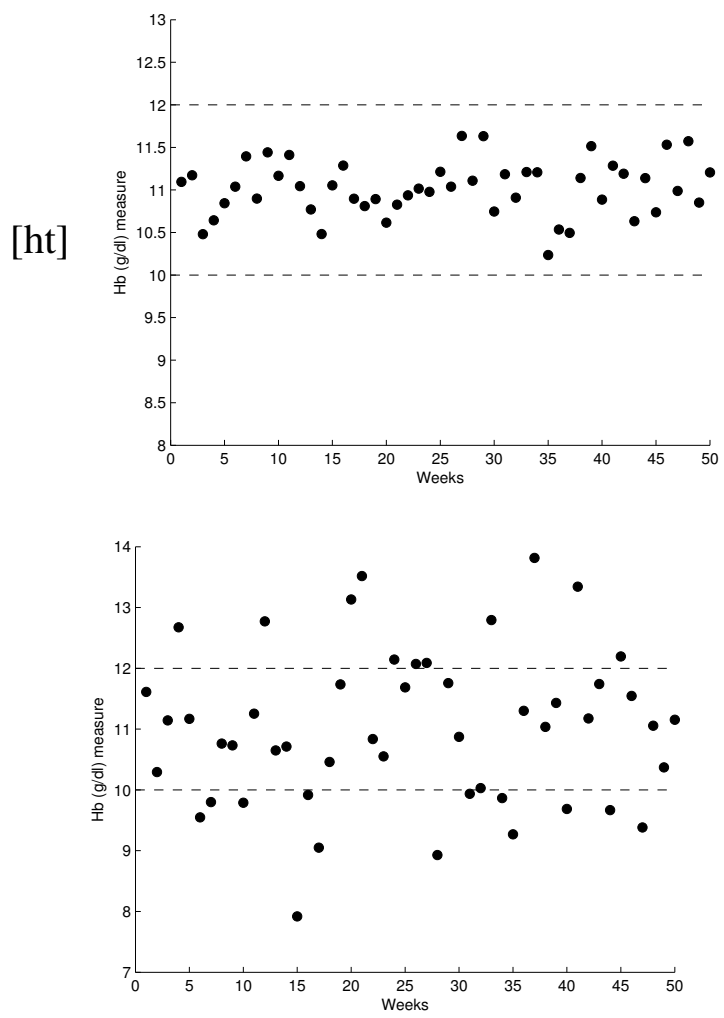

FIGURE 3.1-Simulated Hb concentration of the desired (left) vs actual (right) observations.

\subsection{DEFINITION}

The primary aim of treatment with EPO medication is to achieve and stabilize $\mathrm{Hb}$ concentration of ESRD patients to reduce the need for blood transfusions. The National Kidney Foundation's Dialysis Outcomes Quality Initiative stipulated that patients with such condition maintain $\mathrm{Hb}$ value in the range of 10-12 g/dL [6].

To meet this requirement, several dialysis facilities developed protocols for EPO dosage adjustments [43] that comprise information on FDA approved EPO packet inserts and average patients response to the medication. As such, this dosing strategy tend to address dosage as a population-wide issue and neglect to consider the erythropoietic responses of the few patients with aberrant $\mathrm{Hb}$ responses. Therefore, to achieve an efficient and accurate treatment method, it is important to consider the inherent variability 
within patient $\mathrm{Hb}$ responses to EPO. Figure 3.1 shows the simulated $\mathrm{Hb}$ responses of patients under EPO treatment for both the ideal and actual situation. The figure on the left indicates the desired $\mathrm{Hb}$ response pattern after EPO administration which clearly shows $\mathrm{Hb}$ measurement values are within the targeted range of 10 to $12 \mathrm{~g} / \mathrm{dL}$. By contrast, the figure on the right shows $\mathrm{Hb}$ response values that are typically observed in the clinical settings, which is clearly undesirable. In both figures, dark filled circles are used to represent $\mathrm{Hb}$ measurement and two horizontal lines are used to denote lower and upper limit of desired $\mathrm{Hb}$ response of $10-12 \mathrm{~g} / \mathrm{dL}$. A challenge to EPO therapy is maintaining patient hemoglobin response within the targeted therapeutic range; however, a phenomenon known as hemoglobin cycling, attributed to uncertainty in dosing strategies [44], makes this objective difficult to achieve. An immediate solution to this difficulty is a patient-specific approach to dosage therapy. Optimal dosing decisions for individual patients can be derived using feedback control approaches based on a patientspecific dose-response model estimated from the initial treatment data.

\subsection{ERYThropoiesis DRUG DELIVERY StRATEGY}

Several attempts to automate EPO delivery have already been reported in the literature [45-56]. Bayesian network-based drug delivery optimization was performed with patient population data in [46]. This approach was subsequently enhanced by the Fuzzy rule-based control strategy in [57]. In [53] and [56], Artificial Neural Network models were evaluated in anemia patient model development. However, none of the above techniques is able to provide models based on the individual measurement response data. Few attempts have been made to develop patient-specific models $[47,54,55,58]$. Support Vector Regression [58] and Approximate Dynamic Pro- 
gramming in $[47,54,55]$ have been proposed previously to personalize EPO dosing. In [58], the procedure was mainly to predict input EPO rather than output hemoglobin. This objective is less desirable than predicting hemoglobin output since predicting outputs provides a means to determine the required input value; however, the converse is not true. In $[47,54,55]$ individualized anemia management was attempted; however, the approach was aimed at optimizing the EPO dosage rather than developing individualized patient models. To summarize, none of the above modeling techniques used individual patient measurement data in the model discovery. Furthermore, none considered the effects of patients initial conditions prior to any modeling techniques and more importantly, none was able to deliver models with a quantifiable information on the model uncertainty.

\subsection{Anemia Management as a Feedback Control Problem}

The feedback configuration of Figure 1.1 depicts a possible interaction between a physician and a patient of ESRD where $H b_{d}$ and $H b_{m}$ represent the desired and measured hemoglobin values, respectively. In the figure, EPO values to administer are determined by the physician based on past observations. The objective here is to provide dosing strategies to minimize the error between the measured and desired $\mathrm{Hb}$ value while taking into account the effect of unknown disturbances resulting from changes in fluid volume level, undiagnosed blood loss, acute conditions, and other unknown elements in the overall dynamics. Mathematical description of patient input-output dynamics in the feedback figure can be derived from the EPO and $\mathrm{Hb}$ information using identification techniques. An issue with the standard system modeling methods is data insufficiency common in clinical environment as patients often omit future appointments 
and/or switch medications during treatment procedures. This is because the classical system identification techniques rely on the concept of central limit theory and statistical information where it assumes large measurement sample of the process is available for the model development. A modeling technique that requires less data sample could provide solution to this problem.

The robust identification approach employed here makes no assumption on statistical information on the nature of noise affecting measurements which makes the technique appropriate for personalized modeling. These properties make it capable of providing model based on sparse data. It should be mentioned that model-free based control algorithms are available and can be used [47] to achieve similar goal of stabilizing hemoglobin levels. However, this approach lacks an important benefit mathematical models provide such as a clear relation between EPO to $\mathrm{Hb}$ response as provided in model based approaches. 


\section{CHAPTER 4 SEMI-BLIND ROBUST IDENTIFICATION}

This chapter provides an overview on robust identification of dynamical systems from the worst-case points of view.

\subsection{Robust IDENTIFICATION: AN OVERVIEW}

Estimating mathematical models of an unknown system from measured information is a well known problem dating back to the work of Friedrich Gauss who in 1795 used the least squares approach to calculate the orbits of celestial bodies. In recent times, the least squares methods have expanded into other parametric system estimation techniques such as the output error method (OE), the autoregressive with exogenous in-

put (ARX), etc [59]. The basic requirements of parametric system estimation methods are model structure, including the orders, and measurement noise characteristics. Generally, polynomial functions with unknown parameters are used to define the assumed system structures. Then, measurement data obtained from the physical system are used to estimate these unknown parameters by minimizing certain objective functions usually of $\ell_{2}$-norm types. Clearly, results from this technique depend on the specified model structure. Furthermore, the classical modeling technique does not provide any additional information on quantifying errors due to model structure misspecification. In this chapter, the issues of the parametric system modeling are addressed through a new 
identification technique called the robust identification method which assumes no prior information on the system structure nor assume any statistical information on the noise characteristics affecting measurement samples. Additionally, the technique provides a quantitative norm bounded uncertainty on the derived model.

The robust identification technique is based on dimensionality reduction methods popular in a subfield of mathematics called fractional analysis. The basic idea is to first understand that the unknown system is complex and that no mathematical model can completely represent its dynamics and that any information that is obtained or assumed to be known about the system is incomplete. In addition, the information obtained is corrupted and priced. Therefore, any estimates derived of the system must be accompanied by its associated uncertainty error information. To achieve this, it is generally assumed that the investigated system belongs to a class of systems that share certain basic properties such as time constant, gain, etc. These basic properties are call the a priori information on the system and are used to specify the aforementioned system class structure.

This prior knowledge, when combined, form a set consisting of all possible systems capable of generating the measured a posteriori data. Given the a posteriori information, the aim is to minimize the set consisting of systems that could possibly produce the measured output. The idea is that any system that satisfies both the $a$ priori and the $a$ posteriori information is capable of generating the experimental data provided the $a$ priori information on the system is correctly specified. When such a priori information is validated with new measurement data (a posteriori information), then any model in this set could be selected as a nominal model and its corresponding uncertainty is defined by the radius of the set. Mathematical procedures are provided to test for the consistency between the a priori and a posteriori information. It is shown that when the two information sources are consistent, the maximum possible error that could be obtained as a result of using any model within the set as a nominal model could not produce an error 
more than two times that of any hypothetical optimal interpolatory algorithm.

In classical system identification methods such as the ARX, OE, etc, there exists a familiar dilemma regarding model structure selection. This is related to model complexity. A model structure with a low complexity level has a low probability of identifying the true system model; however, if a model is found, the probabilities of the derived model representing that of the true system's model increase. This increases the variance error. In contrast, a model with a high level of complexity has a high likelihood of including the true system dynamics but it also includes other models that are nearly impossible to be generated by the true system. In this case, the bias error of the model increases. This dilemma is shown in Figure 4.1. In the figure, the dotted line represents the bias error profile which decreases as model complexity level increases. By contrast, the variance error increases with an increase in model complexity as shown with dashed line in the same figure. The solid line profile shows the combined errors of the two error sources while the single dot point in the figure shows the optimal model complexity level. Given a fixed measurement record, there exists an optimum model order that minimizes the total possible error contributions from the two error sources.

\subsection{NONPARAMETRIC IDENTIFICATION IN PHYSIOLOGICAL SYSTEMS}

The requirements that model orders to be specified in advance in the parametric identification techniques as discussed previously can be address by using the nonparametric methods. In the nonparametric approach, the system model structures do not need to be specified a priori; however, this requires that large measurement samples be available to generate meaningful results. Techniques such as the correlation and spectral density functions are commonly used in this technique. The minimal a priori 


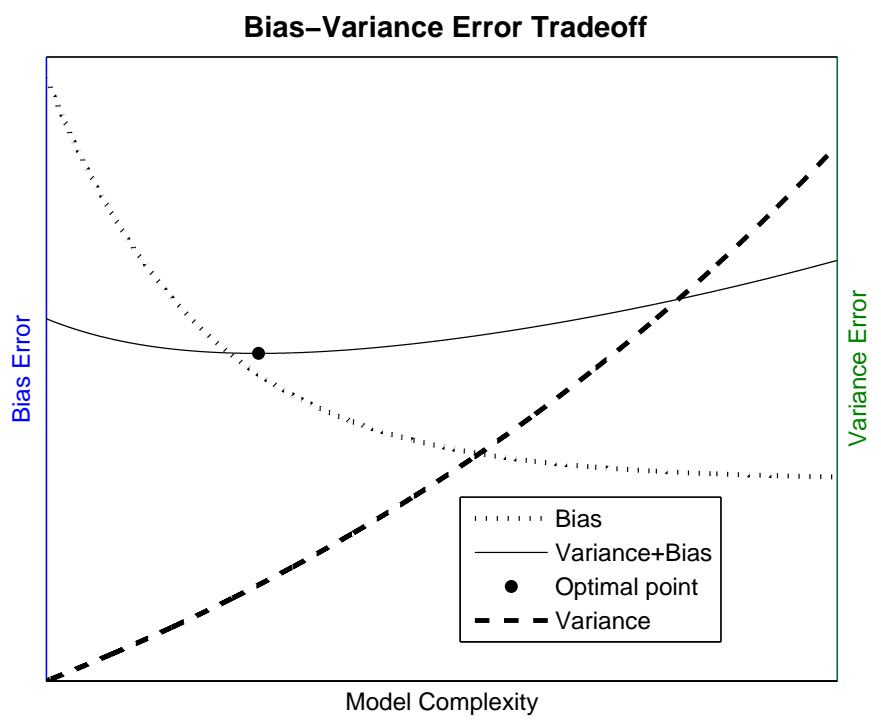

FIGURE 4.1 - Bias-variance tradeoff in model complexity level.

information requirements in the nonparametric technique is extremely useful for modeling physiological systems since, in most cases, the available a posteriori information on these systems is minimal. In a way, the aim is to allow the data to "explain itself". The classical approach of nonparametric identification methods is not suitable for modeling physiological system especially since it requires significant amount of data to be available and that the datasets to be stationary [60]. Identification techniques based on nonparametric approach that requires minimal a priori information as well as small data segment (a posteriori measurements) seems to fulfill the benchmarks for modeling physiological systems. By estimating the system initial conditions using the semi-blind identification methods, the modeling techniques provided meets the needs for nonparametric modeling of physiological systems.

During system modeling, the common assumption is that the system to be identified has zero initial conditions. This assumption simplifies the problem; however, it can limit the performance of the identification results. The goal here is to establish a framework that estimates the nonzero initial conditions of the system using the measurement 
samples. Then, a procedure is provided that determines the worst-case prediction error which is defined as the difference between the model predicted output and the system measurement output. This worst-case prediction error value is an upper bound measure which is always greater than the difference between the two output values. If this condition is ever violated, the implication is that the assumed a priori information on the system is no longer valid and a new a priori information must be assumed.

\subsection{Robust IdentificAtion in Time Domain: An Overview}

System identification is a technique of using finite, partial, and corrupted measurement information to develop mathematical models of the system of interest. Several techniques and procedures are available to achieve this objective. When the ultimate goal of the model is to be able to control the behavior of the system by designing efficient controllers, it is important that the final model is of a low model order, or to have "parsimonious" model orders. This objective is particularly common to the field of systems with electrical and mechanical properties. The parametric identification techniques are appropriate for the modeling of systems with these properties. The other reason for modeling a system is to obtain a better understanding of the internal working behavior of the system. Nonparametric identification techniques are usually used for this modeling objectives that require less a priori knowledge of the system to be stated. Identification methods employing this technique are usually preferable for systems with physiological properties. The robust identification method is a nonparametric approach which assumes very minimal a priori information on the system of interest, yet is capable of providing models that are of much lower order than classical nonparametric identification methods. 
The goal of the robust identification method is to identify the dynamical behavior of a linear time invariant system using noisy measurement data. Assumptions on the noise information affecting measurements is that it is unknown but bounded by a known value. That is the noise is bounded in the $\ell_{\infty}$-norm sense. Furthermore, the system error term is defined in the $\ell_{1}$-norm sense, which provides information on the worstcase identification. The worst-case in this context an identification algorithm that yields a nominal model as well as a quantitative worst-case bound on the model error.

Consider the problem of estimating system $G_{0}$ of Equation (4.1) by using $N$ input-output measurement samples where the variable $y_{k}$ represents output measurement sequences and $u_{k}$ some known input sequences. Variable $\eta_{k}$ represents the noise. In the classical system identification methods, this noise sequence is usually defined by assuming it originates from certain probability distribution functions such as the Gaussian. Though a perfectly valid assumption in certain situations such as cases where there are sufficiently large data available, this assumption on error in measurement fails when the size of data available for the identification is significantly small. In worst-case identification, the only requirement on the noise sequence $\left\{\eta_{k}\right\}$ is that its values are bounded by a known value. Mathematically, this is represented by Equation (4.2) where $\epsilon$ is defined by the upper bound on the measurement error. For most applications of interest, this upper bound value is usually available and provided by the measuring instrument manufacturers.

$$
\begin{gathered}
y_{k}=G_{0}(q, \theta) u_{k}+\eta_{k} \\
\left|\eta_{k}\right| \leq \epsilon_{k}
\end{gathered}
$$

To effect, the worst-case identification approach addresses the same problem from a different perspective by making use of the a priori information available on the noise. From Equation (4.1) and Equation (4.2), Equation (4.3) is derived by a way of 


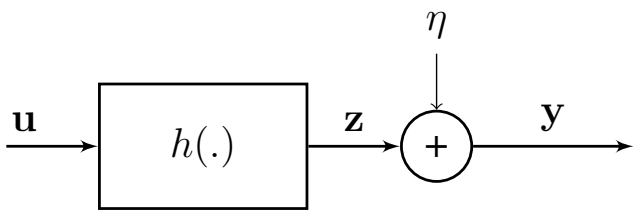

FIGURE 4.2 - An LTI block diagram with input $\mathbf{u}$ and measured output $\mathbf{y}$. Noise signal $\eta$ is unknown but bounded while $\mathbf{z}$ is the unmeasurable signal coming from the system.

algebra. Problem parameter values which satisfies Equation (4.3) contains a convex set called the feasible parameter set [61-64] which is defined as the intersection of parameter values satisfying Equation (4.4).

$$
\begin{gathered}
\left|y_{k}-G_{0}(q, \theta)\right| \leq \epsilon_{k} \\
\theta \in \Theta=\bigcap_{k=1}^{N}\left\{\theta:\left|y_{k}-G(q, \theta) u_{k}\right| \leq \epsilon_{k}\right\}
\end{gathered}
$$

\subsection{NONPARAMETRIC $\ell_{1}$ ROBUST IDENTIFICATION FROM TIME DO- MAIN DATA}

The situation illustrated in the above section assumes the available information on the structure (model order) of the system. Such assumption is applicable for parametric identification methods. For nonparametric identification, the attempt is to produce models with minimal a priori information on the system which effectively allows data to explain itself. This means there should be no assumption on the system order. The robustness aspects of the identification methods implies the derived model should provide a deterministic bound on the modeling error. To achieve this, it is required that there should be no stochastic assumptions on the noise affecting the measurements.

A basic assumption in classical system identification methods is that the system 
of interest has a well defined model structure and the measurement noise is assumed to originate from a certain known probability distribution [59,65-67]. In this case, the only mismatch between the system response values and model predicted values is attributed to the noise. Noise description is only one of the factors affecting the quality of an identified model. An important element is the unrealistic notion that a complex system could be fully explained by a fixed model structure. In reality, the only available information on the system are partial information and a means to quantify the missing piece of information is necessary to provide a meaningful description of the true system behavior. As a result, the robust identification algorithm assumes no such information on the system order nor does it assume any statistical information on noise affecting measurements. The a priori information requirements are very minimum. Such information includes the maximum gain on the system $K>0$, the lower bound on the relative stability margin of the system dynamics $\rho>1$, and an upper bound on the noise in measurements $\epsilon>0$. Given the available a priori information, Figure 4.3 represents a possible impulse response profile. Using the available a priori information, it is assumed the true system belongs to a set defined by the given a priori and a posteriori information (measurement data) provided the a priori information is correct. In this case, the robust identification algorithm provides not only a nominal model but also the uncertainty bound on the model error on the derived nominal model which describes a set containing models capable of explaining the a posteriori information with the given a priori information. Equation (4.5) gives an example of a system set $G(z)$ described by a nominal model $G_{0}$ and additive uncertainty description $\Delta$. Its pictorial representation can be seen in Figure 4.4. Although the uncertainty description of Equation (4.5) can be rearranged to be in the form of Equation (4.2) which describes only the mismatch between measurement values of the system and that of the model, it should be emphasized here that the uncertainty description of Equation (4.5) is a culmination of model error and the measurement error. As such, Equation (4.5) can be described as a norm-bounded 


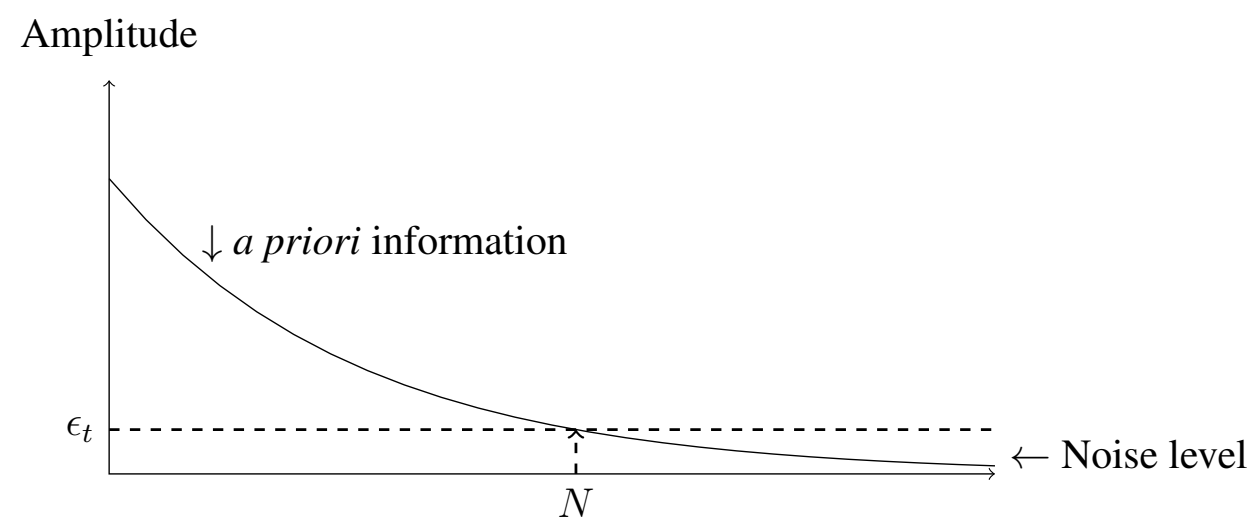

FIGURE 4.3 - Response plot with a priori and a posteriori information.

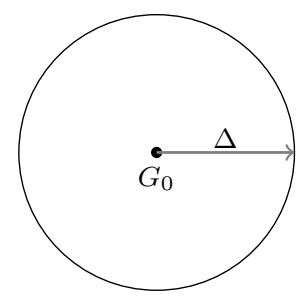

FIGURE 4.4 - Representation of a system described by a nominal model $G_{0}$ and an additive uncertainty bound $\Delta$.

uncertainty as given in Equation (4.6).

$$
\begin{gathered}
G(z)=G_{0}(z)+\Delta \\
\left\|G(z)-G_{0}(z)\right\|_{\infty} \leq \Delta
\end{gathered}
$$

Research direction towards the worst-case identification methods originated when it was realized that the classical system identification techniques were unable to provide the needed requirements for robust controller feedback design synthesis. Aside from the usual requirement of a nominal model common for most advanced controller design based technique, robust controller design technique requires an additional set of information viz uncertainty description on the derived nominal model. Traditional identification methods only provides probabilistic bound, which is a "soft" bound; whereas, 
a "hard" bound model error is required for the robust controller design techniques. To meet this hard bound requirement, intensive research efforts on identification technique which achieve such information requirements. This direction of research effort began with Helmicki and co-authors [14-16, 21, 68, 69] and attracted several other researcher's attention $[18,32,70]$. The research in this area took two directions depending on a posteriori information. The case where measurement data originates from frequency domain leads to $H_{\infty}$ methods $[14,21,35,39,71]$; whose results could be readily applied in $H_{\infty}$ [72] controller design synthesis. When measurement data originates from timedomain experiments, $\ell_{1}$ identification techniques $[21,25,28]$ are used. In the ideal case of full system information including infinite measurement data and zero noise, both techniques provide identical results. However, in the case of non-ideal situations identification technique in one domain may provide an added information not easily accessible in the other domain. As a result, a new identification method that considers both source of information was studied in $[30,32,33,73,74]$. This joint identification method is appropriately termed Mixed Time/Frequency Identification methods which is useful for systems with both source of information available.

\subsubsection{The Robust Identification Problem}

The class of systems considered in this work are causal, single-input-singleoutput linear time-invariant discrete time systems which can be extended to the case of multiple input-multiple output system. Within this framework, a sequence of inputs $u$ in a particular space to sequence of outputs $y$ in another space through a linear operator $\mathcal{S}$ defined by a set of exponentially stable systems whose impulse response is bounded by $K \rho^{-k}, \forall_{k} \geq 0$ where $K>0$ and $\rho>1$. The parameter value $K$ and $\rho$ which provided by the a priori information on the system. Equation (4.7) defines the

z-transform of the impulse response $\left\{h_{k}\right\}_{k=0}^{\infty}$ of a system in system class $\mathcal{S}$ such that 


$$
H \in S=\overline{\mathcal{B}} \mathcal{H}_{\infty, \rho}(K) . \quad H(z)=\sum_{k=0}^{\infty} h_{k} z^{-k}
$$

For the robust identification problem, the input information provided to the robust identification algorithms consists of the class of candidate models, the measurement noise class, and the experimental data. As a result, the robust identification procedure is based not only on the experimental data but also on the available a priori information on the system. To reiterate, the selected system class is a set of exponentially stable systems whose impulse response is defined by $h_{k}=K \rho^{-k}$ where $K$ is the bound on the impulse response and $\rho$ defines its decay rate. Let the output measurement data $y_{k}=H u_{k}+\eta_{k}$ be the collected $N$ samples in response to the input $u_{k}$. Then, the robust identification problem can be stated as follows:

Problem 1. Given the a priori and the a posteriori experimental data, determine

1. whether the a priori and a posteriori information are consistent, i.e decide whether the models in $\mathcal{H}_{\infty, \rho}(K)$ interpolates the given measurement points with error bounded by the a priori error information.

2. If the two sources of information are consistent, then obtain such model as well as the bound on the worst-case identification error.

Definition 1. The a priori information $(\mathcal{S}, \mathcal{N})$ and a posteriori experimental data ( $\left.\left\{u_{k}, y_{k}\right\}_{k=0}^{N-1}\right)$ are consistent if and only if the following set is nonempty

$\mathcal{T} \doteq h \in \mathcal{S} \mid y_{k}=(h * u)_{k}+\eta_{k} \quad$ for some error sequence $\eta_{k} \in \mathcal{N}, k=0,1,2, \ldots, N-1$

If the consistency set $\mathcal{T}$ is empty, then the experimental data sequence $\left\{u_{k}, y_{k}\right\}_{k=0}^{N-1}$ invalidates the a priori assumptions on the system model class. In such case, a new set of a priori information should be considered to find a more suitable model class. Figure 4.5 shows an overview of the robust identification problem. To solve the consistency 


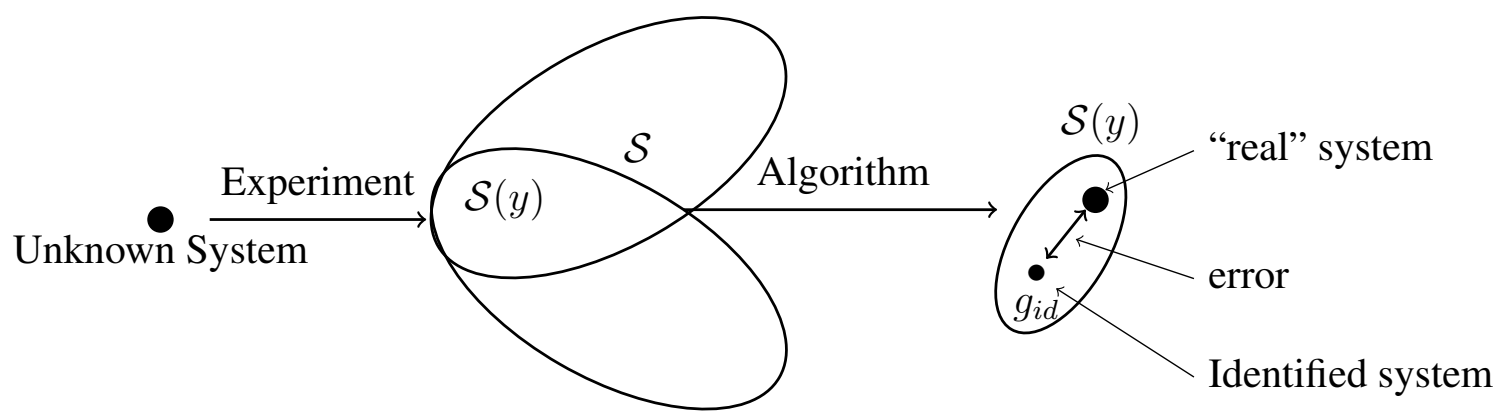

FIGURE 4.5 - An overview of the robust identification technique.

problem, the well known Carathéodory-Fejér Interpolation problem is introduced as below:

Problem 2. Carathéodory-Fejér [35]: Given sequence of complex points, $c_{i}, i=0,1, \ldots, n-$ 1 , determine a function $f \in \hat{\mathcal{B}} \mathcal{H}_{\infty}$ such that

$$
f(z)=c_{0}+c_{1} z+c_{2} z^{2}+\ldots+c_{n-1} z^{n-1}+z^{n} \hat{g}(z),
$$

where $\hat{g} \in \hat{\mathcal{B}} \mathcal{H}_{\infty}$. In other words, the first $n$ Taylor series coefficients of the function $f$ must be determined to match the corresponding complex points such that:

$$
\begin{aligned}
c_{0} & =\frac{f(0)}{1} \\
c_{1} & =\frac{f^{\prime}(0)}{1} \\
c_{2} & =\frac{f^{\prime \prime}(0)}{1 \cdot 2} \\
c_{3} & =\frac{f^{\prime \prime \prime}(0)}{1 \cdot 2 \cdot 3} \\
\vdots & \vdots \\
c_{i} & =\frac{f^{(i)}(0)}{i !}
\end{aligned}
$$

Solution to the Carathéodory-Fejér Interpolation Problem exists if the following Carathéodory-Fejér Theorem holds:

Theorem 1. [35] Given complex set of points, there exists a function $f \in \hat{\mathcal{B}} \mathcal{H}_{\infty}$ such 
that (4.9) is satisfied if and only if

$$
\mathbf{I}-\mathbf{T}_{c}^{H} \mathbf{T}_{c} \geq 0
$$

where $T_{c}$ is an $n \times n$ lower triangular Toeplitz matrix associated with the sequence $\left[c_{0}, c_{1}, \ldots, c_{n-1}\right]$. For equality in (4.11) (rank deficient), the solution to (4.9) is unique; non-unique otherwise. When it is non-unique, the function can be parameterized.

In [35], it is shown that solution to the Carathéodory-Fejér Interpolation Theorem exists if and only if there exists a vector $\mathbf{h}=\left[h_{0}, h_{1}, h_{2}, \ldots, h_{N-1}\right]$ such that the following Linear Matrix Inequalities (LMIs) holds:

$$
\begin{gathered}
M(\mathbf{h})=\left[\begin{array}{cc}
K R^{-2} & \left(T_{h}^{N}\right)^{T} \\
T_{h}^{N} & K R^{2}
\end{array}\right] \geq 0 \\
\left|y-T_{u}^{N} h\right| \leq \epsilon
\end{gathered}
$$


where $R=\operatorname{diag}\left[\begin{array}{lllll}1 & \rho & \rho^{2} & \ldots & \rho^{N-1}\end{array}\right]$ and

$$
\begin{aligned}
& T_{u}^{N}=\left[\begin{array}{cccc}
u_{0} & u_{1} & \ldots & u_{N-1} \\
0 & u_{0} & \ldots & u_{N-2} \\
\vdots & \vdots & \ddots & \vdots \\
0 & 0 & \ldots & u_{0}
\end{array}\right] \\
& T_{h}^{N}=\left[\begin{array}{cccc}
h_{0} & h_{1} & \ldots & h_{N-1} \\
0 & h_{0} & \ldots & h_{N-2} \\
\vdots & \vdots & \ddots & \vdots \\
0 & 0 & \ldots & h_{0}
\end{array}\right] \\
& \mathbf{y}=\left[y_{0}, y_{1}, \ldots, y_{N-1}\right]^{T} \\
& \mathbf{u}=\left[u_{0}, u_{1}, \ldots, u_{N-1}\right]^{T}
\end{aligned}
$$

\subsubsection{Parametric Inclusion}

As mentioned in the previous section, robust identification procedures that are solely based on nonparametric assumptions may yield conservative results especially if parts of the model have a clear parametric structure. Less conservative bound can be derived if information on the parametric portion is included in the identification procedure. In that case, the new a priori information on the system class becomes

$$
\mathcal{S} \doteq\{G(z)=H(z)+P(z)\}
$$

where $H(z)$ is the nonparametric portion of the system as defined in the section above and $P(z)$ is the known parametric portion of the system, respectively. Furthermore, it is 
assumed that the parametric portion of the system, $P(z)$, belongs to some affine set $\mathcal{P}$ of the form described in Equation (4.15)

$$
\mathcal{P} \doteq\left\{P(z)=p^{T} G_{p}(z), p \in \mathcal{R}^{N_{P}}\right\}
$$

where $p \in \mathcal{R}^{N_{P}}$ is some unknown vector of some a priori known component $G_{p}$ and $N_{p}$ is the number of unknown $p$ parameters to be determined. The inclusion of parametric information does not significantly change LMI feasibility problem defined in (4.12). In fact, Equation (4.16) shows this modification where $(P)_{k} \doteq\left[g_{k}^{1} g_{k}^{2} \ldots g_{k}^{N_{p}}\right], g_{k}^{i}$ denotes the $k^{\text {th }}$ Markov parameter of the $i^{\text {th }}$ transfer function of the parametric portion and $h_{k}$ are the Markov parameters of the nonparametric portion.

$$
\begin{aligned}
M_{R}(\mathbf{h})= & {\left[\begin{array}{cc}
K R^{-2} & \left(T_{h}^{N}\right)^{T} \\
T_{h}^{N} & K R^{2}
\end{array}\right] \geq 0 } \\
& \left|y-\left(T_{u}^{N} p P+T_{u}^{N} h\right)\right| \leq \epsilon
\end{aligned}
$$

In both purely nonparametric and the combined parametric/nonparametric cases, the system class $\mathcal{S}$ is assumed to contain the true system producing the a posteriori measurement data. The following information shows the procedures used to determine the nominal model from the a priori model class. This is performed by parameterizing the set $\mathcal{S}$ with a free parameter $Q(z) \in \mathcal{B H}_{\infty}$. A simple such parameter of choice is the zero parameter, thus $Q(z)=0$ leads to the central model $S_{\text {central }}=H_{0}(z)+\mathbf{p}^{T} \mathbf{G}_{p}(z)$ where explicit state space realization of $H_{0}(z)$ can be determined as follows [75]: 


$$
\begin{aligned}
& H(z)=\left[\begin{array}{c|c}
A_{H} & B_{H} \\
\hline C_{H} & D_{H}
\end{array}\right]=C_{H}\left(z I-A_{H}\right)^{-1} B_{H}+D_{H} \\
& A_{H}=A-\left[C_{-}^{T} C_{-}+\left(A^{T}-I\right) M_{R}\right]^{-1} C_{-}^{T} C_{-}(A-I) \\
& B_{H}=-\left[\left(A^{T}-I\right) M_{R}+C_{-}^{T} C_{-}\right]^{-1} C_{-} \\
& C_{H}=C_{+}\left[\left(A^{T}-I\right) M_{R}+C_{-}^{T} C_{-}\right]^{-1} C_{-}^{T} C_{-}(A-I)-C_{+}(A-I) \\
& D_{H}=C_{+}\left[\left(A^{T}-I\right) M_{R}+C_{-}^{T} C_{-}\right]^{-1} C_{-}^{T}
\end{aligned}
$$

where

$$
A=\left[\begin{array}{cc}
0 & I_{N \times N} \\
0 & 0
\end{array}\right], C_{-}=\left[\begin{array}{llll}
1 & 0 & \ldots & 0
\end{array}\right], C_{+}=\frac{\mathbf{h}^{T} R}{K}
$$

and $M_{R}$ is defined in Equation (4.16). 


\subsection{SEMI-BLIND Robust IdentificAtion Of LTI Systems}

Until now, system $G$ is identified while assuming a zero initial conditions on its dynamics. However, such assumption on systems that are already in progress is erroneous and could introduce artificially large identification errors on the model predicted values. In this section, a recently discovered robust identification technique called semiblind robust identification is introduced. With this new technique, the robust identification method attempts to provide information on the system initial conditions prior to the identification process. To incorporate the effects of the initial conditions on the system, a new set of parameter is introduced which the robust identification algorithm must find in addition to the solutions to the original problem of impulse response estimates formulated as follows $[75,76]$ :

Problem 3. Given:

1. an unknown plant $G(z)=H(z)+P(z)$, where $H(z)$ and $P(z)$ are the nonparametric and parametric portion of system $G(z)$, respectively,

2. a priori sets of candidate models and noise $(\mathcal{S}, \mathcal{N})$,

3. a characteristics of the set of the past input sequence $\mathbf{u}^{-} \doteq\left\{u_{k}, k=-1,-2, \cdots-\right.$ $N\} \in \mathcal{U}^{-}$applied prior to time $t_{0}$,

4. a finite set of input $\mathbf{u}=\left\{u_{0}, u_{1}, \ldots, u_{N-1}\right\}$ and output data $\mathbf{y}=y_{0}, y_{1}, \ldots, y_{N-1}$, corrupted by additive measurement noise $\eta$.

Determine whether the set $\mathcal{T}(\mathbf{y})$ is non-empty, where

$\mathcal{T}(\mathbf{y})=\left\{G \in \mathcal{S}: y_{k}=\left(\mathbf{T}_{g}^{N} \mathbf{u}^{+}\right)_{k}+\left(\boldsymbol{\Gamma}_{g}^{N} \mathbf{u}^{-}\right)_{k}+\eta\right.$ for some sequence $\eta \in \mathcal{N}$, and $\left.\mathbf{u}^{-} \in \mathcal{U}^{-}\right\}$

Then for a nonempty set $\mathcal{T}(\mathbf{y})$, find the model $G \in \mathcal{T}(\mathbf{y})$ 
The set $\mathcal{T}(\mathbf{y})$ contains all systems $G$ compatible with the given a priori information and a posteriori measurement data. In equation (4.18), the first part corresponds to the response of $G$ to the input $\mathbf{u}$ while the second part corresponds to the portion of system $G$ responsible for inputs prior to the identification. $\mathbf{T}_{g}^{N}$ and $\Gamma_{g}^{N}$ are the Toeplitz and the Hankel matrix operators associated with current and past system dynamics, respectively. To represent the dynamics of the system prior to the identification procedure, we first replace the system past inputs convolved with the system dynamics by some value $x_{k}$ where $x_{k}=\left(\boldsymbol{\Gamma}_{g}^{N} \mathbf{u}^{-}\right)_{k}$ for some $u^{-} \in \mathcal{U}$. Assuming, as part of the a priori information, we have available a bound on the input $\mathcal{U}=\mathcal{B} \ell^{p}\left(K_{u}\right)$ and a bound on the system prior to the identification process $\left\|\Gamma_{g}\right\|_{\ell^{p} \rightarrow \ell^{\infty}} \leq \gamma$. With this new information, the new problem can be stated as follow:

Problem 4. [76] Given an unknown plant, a priori sets of candidate models and noise $\operatorname{set}(\mathcal{S}, \mathcal{N})$ and a finite set of samples $u$ to the plant and its corresponding output $y$ corrupted by additive measurement noise $\eta$, find a model $g$ compatible with both the a priori information and a posteriori experimental data such that $g \in \mathcal{T}(y)$ where

$$
\begin{gathered}
\mathcal{T}(\mathbf{y}) \doteq\left\{y_{k}=\sum_{i=0}^{k} G_{i} u_{k-i}+C_{G} A_{G}^{k-1} \mathbf{x}_{\mathbf{0}}+\eta\right. \\
k=0,1, \ldots N-1 ; \text { for some sequence } \eta \in \mathcal{N}\}
\end{gathered}
$$

where

$$
G \equiv\left(\begin{array}{c|c}
A_{G} & B_{G} \\
\hline C_{G} & D_{G}
\end{array}\right) \quad g_{0}=D_{G} ; g_{i}=C_{G}\left(A_{G}\right)^{i-1} B_{G}
$$

Solution to the above Equation (4.19) involves solving a Bi-Affine Matrix Inequalities problem $x_{0}$ and $g_{i}$, which is known to generally be non-convex and NP-hard optimization problem. However, it has been shown [77] that such problem can be reduced to a tractable problem and solved with a minimal relaxation on the system. This 
convex relaxation approach is valid because of the controllability and observability assumptions on the system. The convex relaxation problem is stated and summarized below:

Problem 5. [76] Given an unknown plant, the a priori candidate models set, past inputs and noise set $\mathcal{S}, \mathcal{U}, \mathcal{N}$ and a finite set of samples of the input and output $\mathbf{u}, \mathbf{y}$ of the system in [0,N-1], find a model $g \in \mathcal{T}$, where

$$
\begin{array}{r}
\mathcal{T}(\mathbf{y})=\left\{g \in \mathcal{S}: y_{k}-\left(\mathbf{T}_{g}^{N} \mathbf{u}^{+}\right)_{k}-x_{k} \in \mathcal{N}\right. \\
\text { for some sequence } \\
\left.|x|_{k} \leq \gamma K_{u}(k=0,1, \ldots, N-1)\right\}
\end{array}
$$

The problem stated above has solution if the following set of LMIs in $h$ and $x$ are feasible

\section{Proposition 1.}

$$
\begin{aligned}
M(\mathbf{h})= & {\left[\begin{array}{cc}
K R^{-2} & \left(T_{h}^{N}\right)^{T} \\
T_{h}^{N} & K R^{2}
\end{array}\right] \geq 0 } \\
& \left|y-\left(T_{u}^{N} p P+T_{u}^{N} h\right)-x\right| \in \mathcal{N} \\
& -\gamma K_{u} \leq x \leq \gamma K_{u}
\end{aligned}
$$

where $\gamma, K_{u}, p$, and $P$ represent system gain, maximum input values, affine parameter, and the parametric portion of the system, respectively. Additionally, the $T_{u}^{N}$ and $T_{h}^{N}$ are the $N$ Toeplitz matrix formed with the input and the system impulse responses, respectively.

Proof. See [78] 


\section{CHAPTER 5}

\section{SEMI-BLIND ROBUST IDENTIFICATION APPROACH TO ANEMIA MANAGEMENT PROBLEM}

In this chapter the semi-blind robust identification technique introduced in the previous chapter is applied to establish individualized models for patients of anemia based on response samples.

\subsection{INDIVIDUALIZING ANEMIA PATIENTS}

The Bayesian approach to drug dosing has extensively been used to optimize the performance erythropoietin in anemia management $[46,79]$. The basic requirement of this technique is that the a priori assumption on certain parameters must be specified in advance, originating from a known probability distribution function. Clearly, the accuracy of these parameter distributions is a major challenge [80]. Additionally, the very nature of stochastic a priori assumptions limits one's ability to obtain hard bound on modeling error to concretely determine when a given system model is no longer suitable for the intended application. A technique that is capable of providing such hard bound is desirable in clinical settings since it provides a mechanism to invalidate any $a$ priori assumptions on the existing patient model.

In the literature on ESRD management with EPO medication, patients are classified as either a poor or normal responder to treatment where the later group are 
patients that respond well to an average dose, defined as 12,000 Units per week, of EPO $[47,57,81]$. In contrast, patients belonging to the poor responder group respond poorly to treatment and pose a major challenge to practitioners to strategize a consistent treatment methods. To make use of the semi-blind identification framework proposed in the previous chapter, it is necessary to characterize the past input values into a set $\mathcal{U}_{-}$ which contains all possible EPO values prior to the identification process. In principle, this set could be defined as EPO values less than or equal to the maximum administered EPO to a given patient such that $u^{-} \in \mathcal{U}_{-}$, where $u^{-} \leq|u|_{\text {max }}$; however, this assumption may be too coerced and conservative to account for possible intervention events such as blood loss and hemodialysis treatments. Therefore, a proper choice for bound on this set is the change in the EPO values; consequently, the modeling problem then becomes identifying an operator $S$ mapping the change in EPO, $u_{k}=E P O_{k}-E P O_{k-1}$ to $H b_{k+1}$. From this definition, it should be emphasized that the a priori information on the system should include an integrator which can be considered as a parametric contribution of the system. Figure 5.2 provides a visual view of this integrator where $\mathbf{u}_{\mathbf{k}}$ is the difference in EPO values and $\mathbf{H b}_{\mathbf{k}+\mathbf{1}}$ and $p$ is a an unknown constant parameter of the parametric portion of the model $P(z)$. To improve the performance of the optimization process, the input data were uniformly rescaled to the interval of $[-1,1]$.

Equation (5.1) provides a summary of both the a priori system and noise sets where $P(z)$ and $H(z)$ represent the parametric and nonparametric portions of the system, respectively.

$$
\begin{aligned}
& \mathcal{S}=\left\{G(z)=H(z)+P(z), \quad P(z)=p \frac{z}{z-1}, \quad H \in \mathcal{B H}_{\infty, \rho}(K)\right\} \\
& \mathcal{N}=\left\{\eta \in \ell^{\infty}:|\eta| \leq \epsilon\right\}, \quad \epsilon=0.31 \\
& \mathcal{U}_{-}=\left\{u \in \ell^{\infty}:\left|u_{k}\right| \leq u_{\max }\right\}
\end{aligned}
$$

An error bound of $\epsilon=0.31$ is selected based on measurement error information obtained from the hematocrit measuring device used at the dialysis facility [82] and $u_{\max }$ 


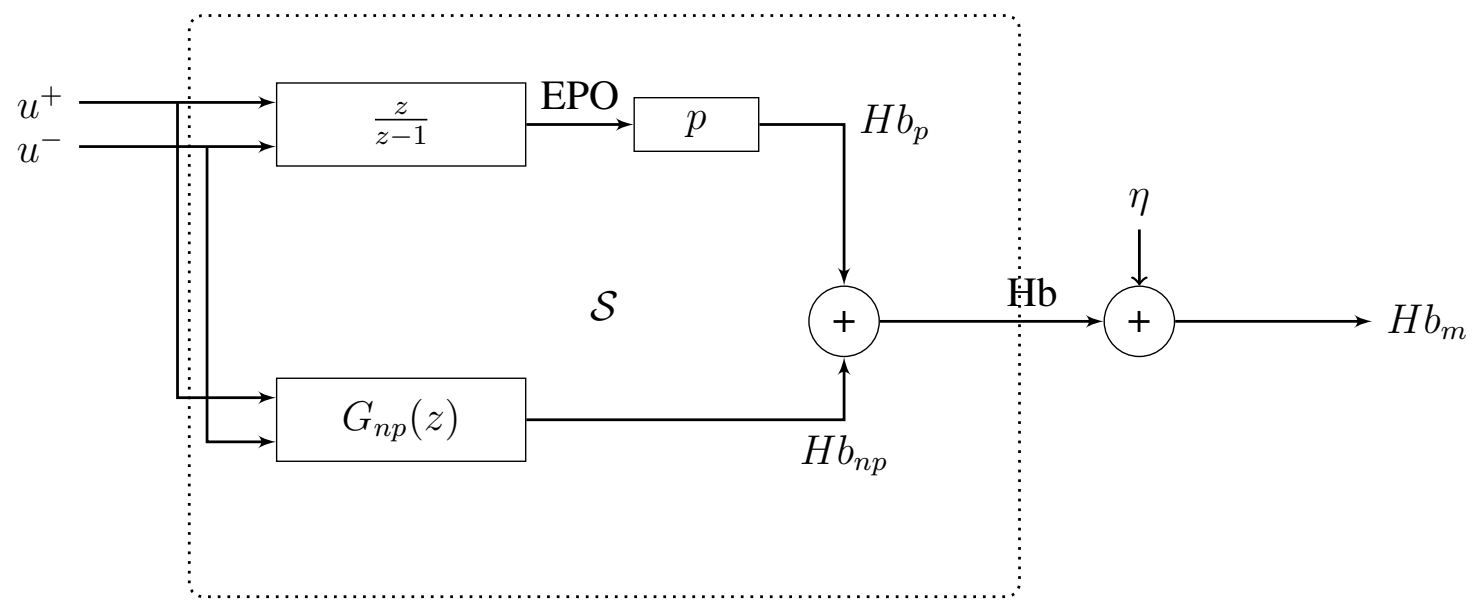

FIGURE 5.1-Anemia Patient model connected with semi-blind robust identification configuration

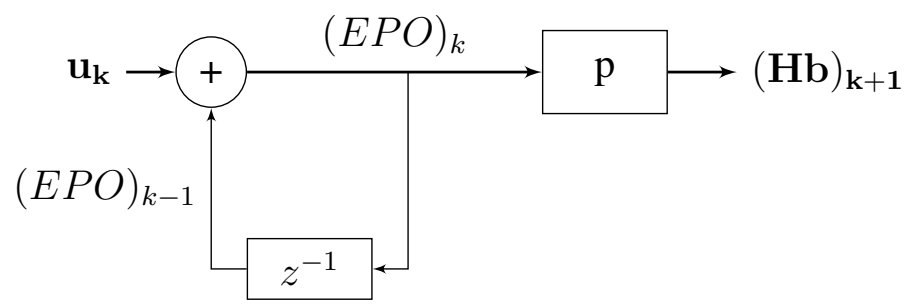

FIGURE 5.2 - This figure represents the parametric portion of the system. 


\begin{tabular}{|c|c|c|c|c|}
\hline Patient\# & $K$ & $\rho$ & $N$ & Respondent Group \\
\hline 1 & 0.295 & 1.0604 & 15 & $\times$ \\
28 & 0.280 & 1.0900 & 17 & $\times$ \\
30 & 0.266 & 1.0700 & 18 & $\times$ \\
6 & 0.280 & 1.0214 & 15 & $\checkmark$ \\
37 & 0.176 & 1.0870 & 15 & $\checkmark$ \\
\hline
\end{tabular}

TABLE 5.1

Some a priori parameter information for the selected patients of poor responders patients \#1, \#28, and \#30 and normal responders, patients \#6 and \#37. $N$ represents the number of data points used in the identification and we indicated with a check mark whether patient belongs to a responder or non-responder groups.

is the maximum input value that is determined from the individual patient data. Table 5.1 summarizes the remaining a priori information on the selected patients. The gain, $K$, is determined by the LMIs of Equation (4.16) and the decay rate values, $\rho$, are heuristically determined to obtain suitable results. Though there may exist some optimization algorithm to determine the optimal decay rate values, $\rho$, such procedure is not known to us at the time of this study.

Fifty-six clinical patient data consisting of weekly information on EPO medication and $\mathrm{Hb}$ measurements are obtained from the University of Louisville Kidney Disease Program for this analysis. Generally, doses are administered three times per week whereas $\mathrm{Hb}$ measurements are taken weekly. Since the identification process requires an input/output pairs of the same length, a preprocessing of the data is performed by summing up the total amount of dosage administered in a given week to a corresponding $\mathrm{Hb}$ measurement values. In addition, to have sufficient data for both identification and validation, a minimum of fifteen data length is set. That is after performing the preprocessing, patients with less than fifteen input/output pairs are omitted; as such, six of the total patients are omitted in the study. Using the aforementioned semi-blind robust 
identification procedure, we obtained models ranging from first to third order models for the remaining fifty patients. Results from five patients are summarized below. Three of which are considered to belong to the poor responder group (Figures 5.3, 5.4, and 5.5) and two normal responder group (Figures 5.6 and 5.7). A one-step-ahead prediction is performed in predicting the hemoglobin levels in each case. Running the proposed algorithm for the measured $\mathrm{Hb}$ data and the corresponding EPO and after performing model reduction by eliminating uncontrollable and unobservable states, transfer function equations are derived as shown in Equations (5.2) through (5.6) for the selected patients where a superscript is used to indicate patient ID number. For example the $G^{1}(z)$ of Equation (5.2) shows the transfer function of patient number one.

$$
\begin{gathered}
G^{1}(z)=\frac{1.03 z^{3}+1.398 z^{2}+0.841 z-0.0413}{z^{3}+0.317 z^{2}-0.501 z-0.816} \\
G^{28}(z)=\frac{0.516 z^{2}-.361 z+0.472}{z^{2}-.368 z+.632} \\
G^{30}(z)=\frac{1.018 z+0.244}{z-1} \\
G^{6}(z)=\frac{0.824 z^{2}+0.832 z+0.162}{z^{2}-0.114 z-0.886} \\
G^{37}(z)=\frac{1.046 z+0.080}{z-1}
\end{gathered}
$$

Using the time delay properties of the z-transform $\mathcal{Z}^{-1}\left\{z^{n} G(z)\right\}=g_{k+n}$, Equations 
(5.2) through (5.6) are equivalently represented as follows:

$$
\begin{array}{rr}
H b_{k+3}^{1}= & -0.317 H b_{k+2}+0.501 H b_{k+1}+0.816 H b_{k} \\
& +1.030 u_{k+3}+1.398 u_{k+2}+0.841 u_{k+1}-0.0413 u_{k} \\
H b_{k+2}^{28}= & 0.368 H b_{k+1}-0.632 H b_{k} \\
& +0.516 u_{k+2}-0.361 u_{k+1}+0.472 u_{k} \\
H b_{k+1}^{30}= & H b_{k}+1.018 u_{k+1}+0.244 u_{k} \\
H b_{k+2}^{6}= & 0.114 H b_{k+1}+0.886 H b_{k} \\
& +0.824 u_{k+2}+0.832 u_{k+1}+0.162 u_{k}
\end{array}
$$

where $H b_{k}$ and $u_{k}$ are the $\mathrm{Hb}$ and the change in EPO at time $k$, respectively.

\subsubsection{Performance Measure}

The forecasting power of the proposed approach can be seen in Figures 5.3 to 5.7

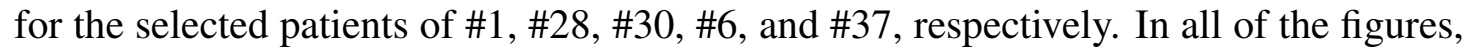
"+" signs are used to denote experimental data used during the identification stage and

“*” signs are used to indicate the data used for forecasting. Predicted output values of the model are denoted by "o". In addition, a dotted vertical line is used to demarcate data used during the identification process and those used for validation. Maximum and average prediction errors defined in Equations (5.12) and (5.13), respectively, are used as a performance measure of the models. In these equations $y_{\text {data }}$ and $y_{\text {pred. }}$ are the measured and predicted $\mathrm{Hb}$ values, respectively. 


\begin{tabular}{|l|c|c|}
\hline Patient\# & error $_{\max }$ & error $_{\text {rms }}$ \\
\hline 1 & 1.07 & 0.28 \\
28 & 0.68 & 0.37 \\
30 & 1.35 & 0.40 \\
6 & 0.65 & 0.26 \\
37 & 0.99 & 0.35 \\
\hline
\end{tabular}

TABLE 5.2

Average and maximum prediction error for the selected patients

$$
\begin{gathered}
\mid \text { error }_{\max }=\left|y_{\text {data }}(i)-y_{\text {pred. }}(i)\right|_{\infty} \\
\text { error }_{R M S}=\sqrt{\frac{\sum_{i=1}^{N}\left(y_{\text {data }}(i)-y_{\text {pred. }}(i)\right)^{2}}{N}}
\end{gathered}
$$

Table 5.2 provides a summary of both error types for the selected patients. While the maximum prediction error values may seem high for certain patients, it should be emphasized that there were no patient specific attributes such as weight, race, gender, etc. are considered at the modeling process. From the root means square prediction error, however, one can conclude the computed error values are acceptable given the width of the target range $(11-12 \mathrm{~g} / \mathrm{dL})$.

Figure 5.8 provides a summary of maximum prediction error for the remaining patient data. In the figure, star " $\star$ " symbol is used to represent the absolute predicted values for patients with sufficient data for both prediction and validation while circles are used to denote patients with insufficient data for prediction and forecasting as described above. 

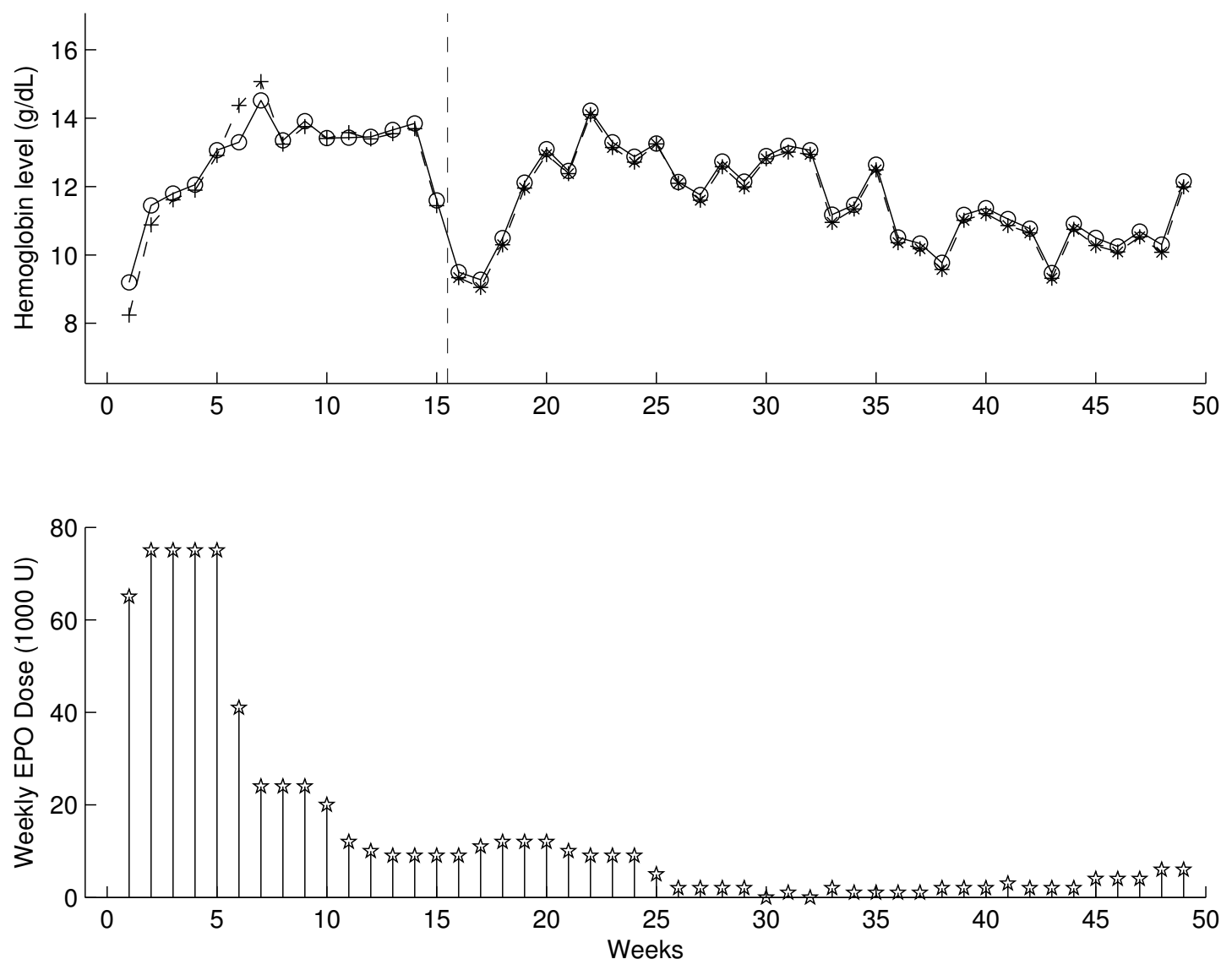

FIGURE 5.3-Semi-blind robust identification results for patient \#1. [Top] Hb prediction with a vertical line used to demarcate data used for identification and for forecasting. A (+) is used to denote experimental data used during the identification and (*) is used to denote data not used in the identification. (o) symbol is used to indicate predicted results with $3^{\text {rd }}$ order model. [Bottom] Administered erythropoietin dose in x1000 Units per week. 

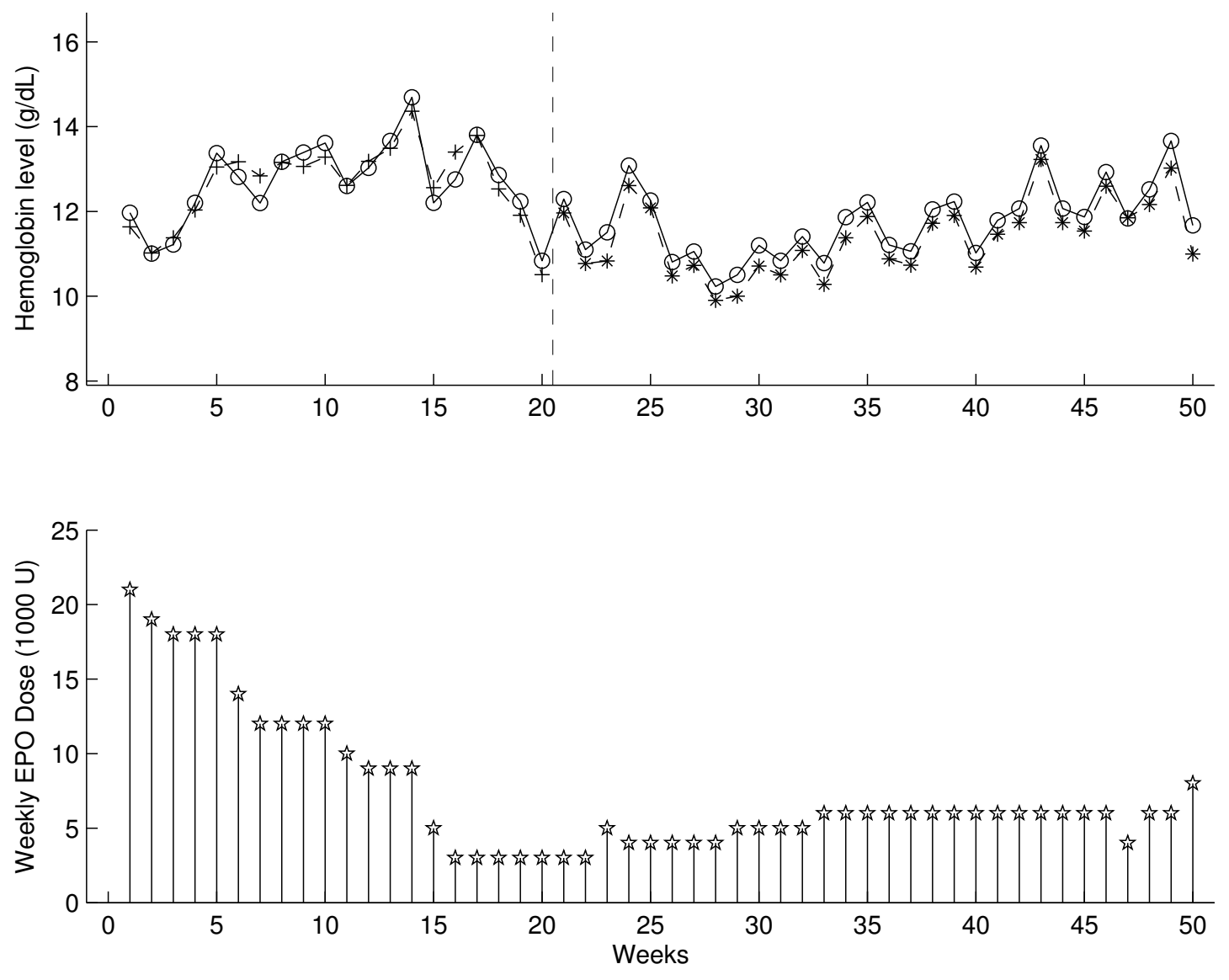

FIGURE 5.4-Semi-blind robust identification results for patient \#28. [Top] Hb prediction with a vertical line used to demarcate data used for identification and for forecasting. A (+) is used to denote experimental data used during the identification and $(*)$ is used to denote data not used in the identification. (o) symbol is used to indicate predicted results with $2^{\text {nd }}$ order model. [Bottom] Administered erythropoietin dose in x1000 Units per week. 

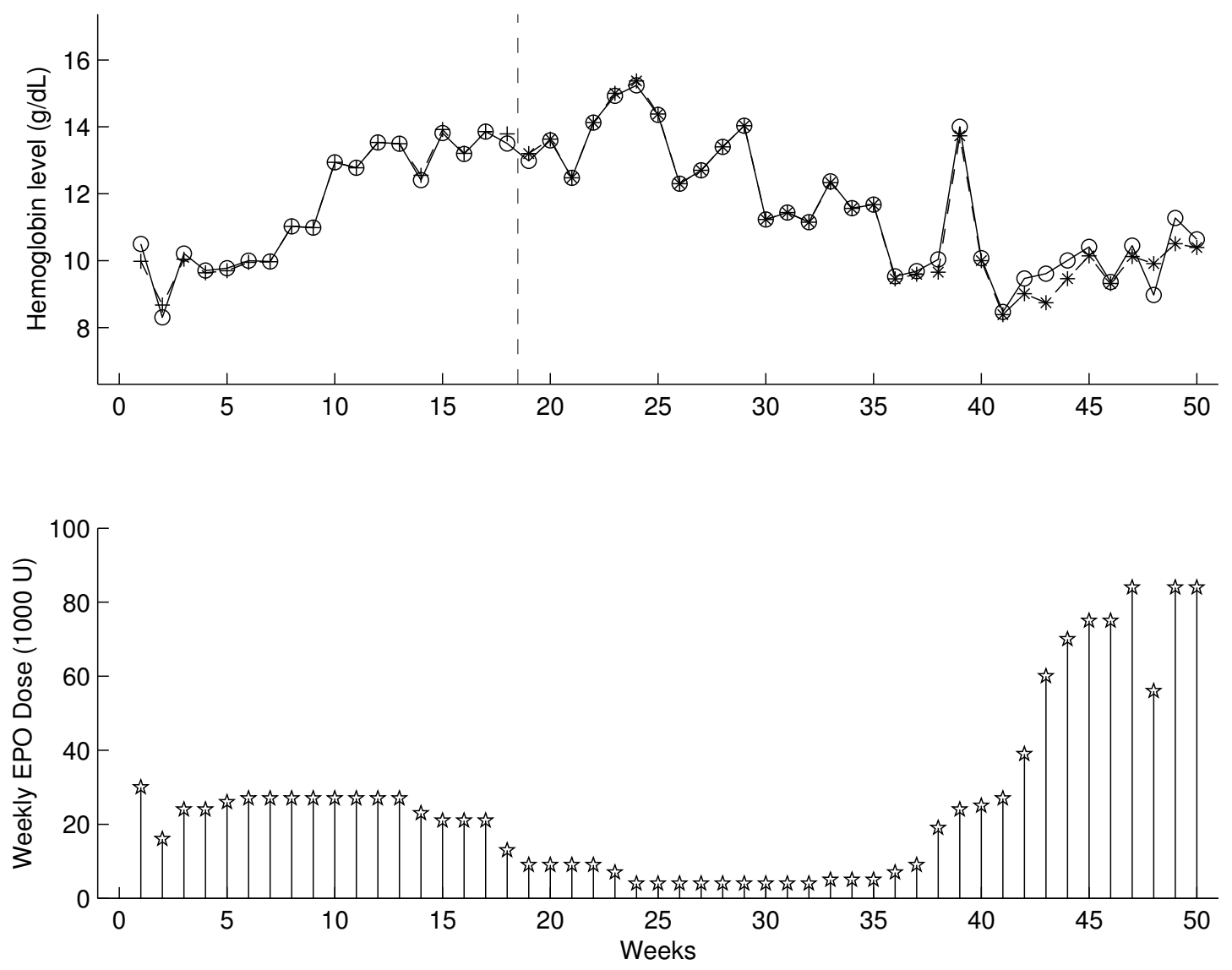

FIGURE 5.5 - Semi-blind robust identification results for patient \#30. [Top] Hb prediction with a vertical line used to demarcate data used for identification and for forecasting. A (+) is used to denote experimental data used during the identification and (*) is used to denote data not used in the identification. (o) symbol is used to indicate predicted results with $1^{\text {st }}$ order model. [Bottom] Administered erythropoietin dose in x1000 Units per week. 

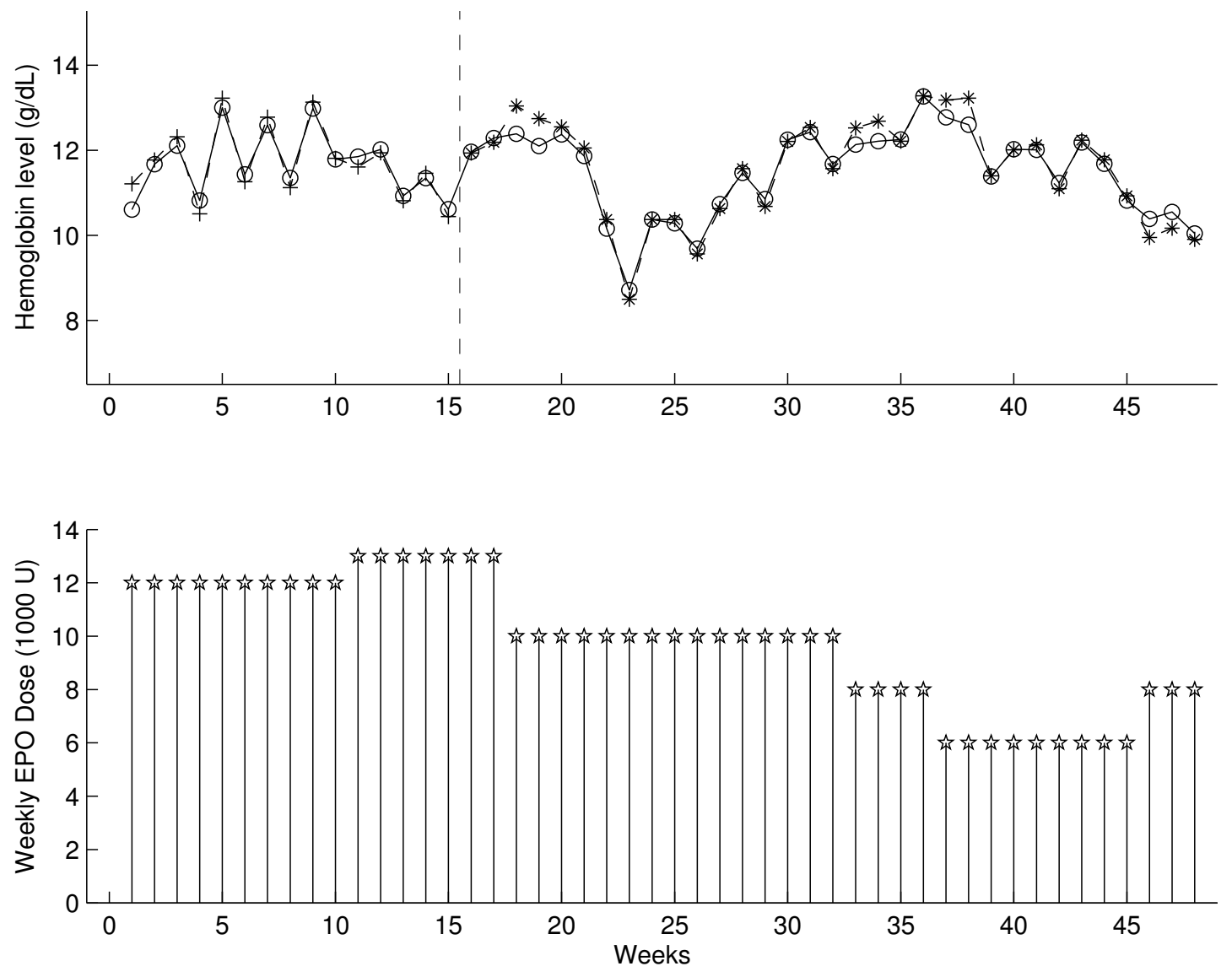

FIGURE 5.6-Semi-blind robust identification results for patient \#6. [Top] Hb prediction with a vertical line used to demarcate data used for identification and for forecasting. A (+) is used to denote experimental data used during the identification and (*) is used to denote data not used in the identification. (o) symbol is used to indicate predicted results with $2^{\text {nd }}$ order model. [Bottom] Administered erythropoietin dose in x1000 Units per week. 

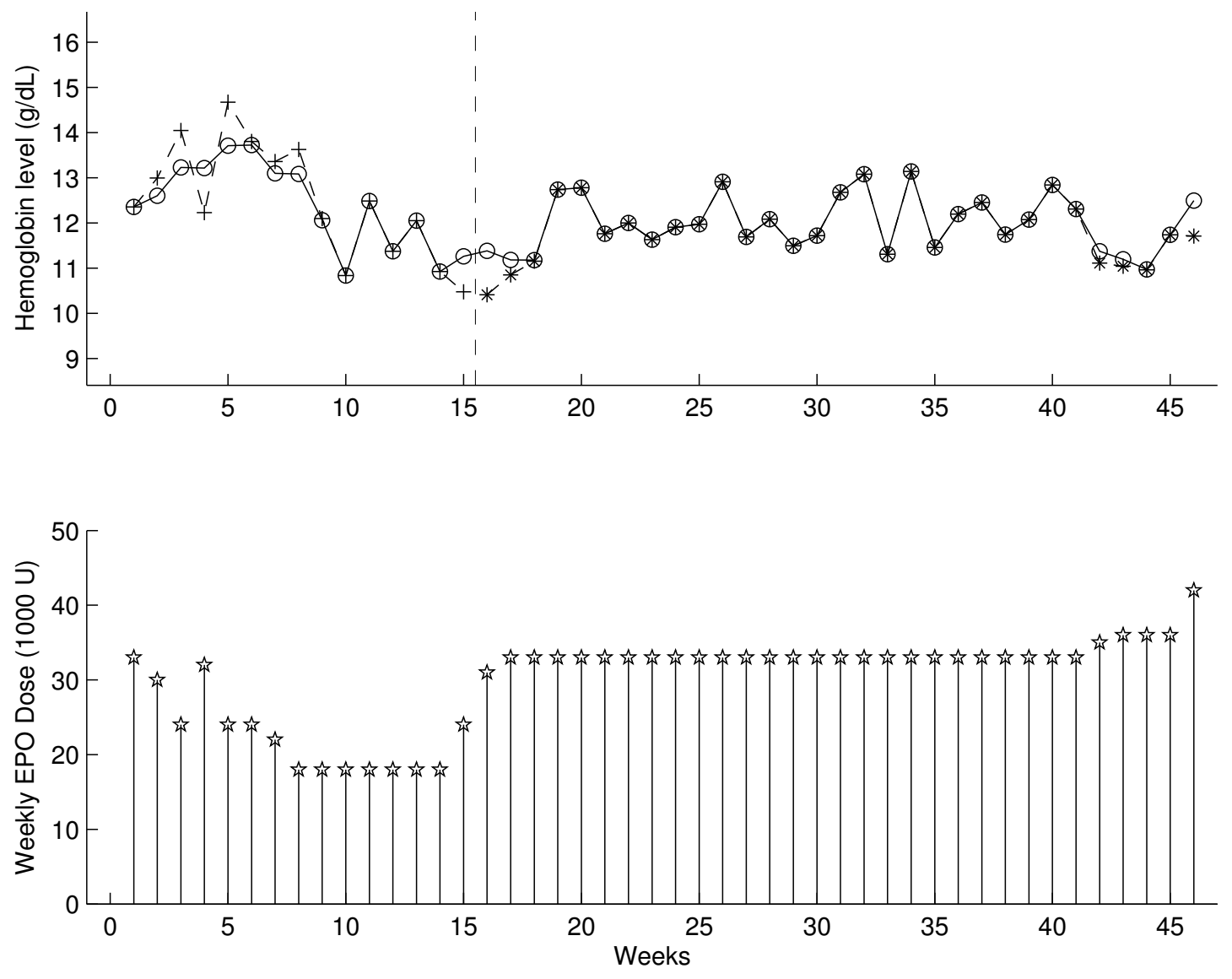

FIGURE 5.7-Semi-blind robust identification results for patient \#37. [Top] Hb prediction with a vertical line used to demarcate data used for identification and for forecasting. A (+) is used to denote experimental data used during the identification and $(*)$ is used to denote data not used in the identification. (o) symbol is used to indicate predicted results with $1^{\text {st }}$ order model. [Bottom] Administered erythropoietin dose in x1000 Units per week. 


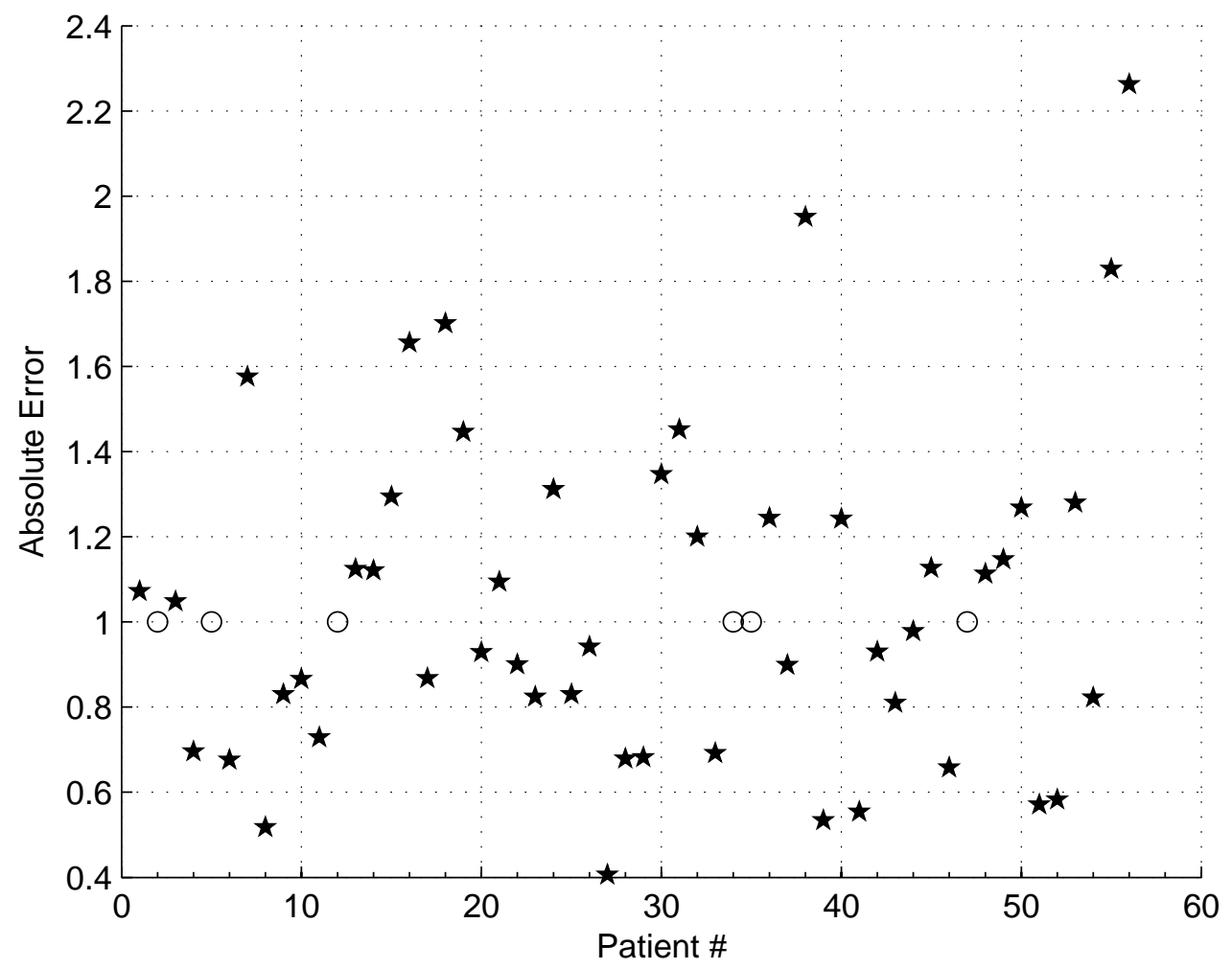

FIGURE 5.8 - Maximum prediction error for all patients from the semi-blind identification technique. 


\subsection{Worst-CASE PREDiction ERror Bounds}

The algorithm introduced in the last section is an interpolation algorithm which implies the true system model belongs to the consistency set $\mathcal{T}(y)$ specified by the $a$ priori information and the a posteriori measurements, provided the a priori information is correctly specified. By using the derivations in [75-77], summarized here, one can state that given the first $N$ measurements such that $\left\{y_{i}\right\}_{0}^{N-1}$, the error bound on the next output values at $t=N$ is defined as:

$$
\begin{aligned}
\left|e_{N}\right| & \leq \sup _{g_{1}, g_{2} \in \mathcal{T}(y)}\left|\left(T_{g_{1}}-T_{g_{2}}\right) u^{+}+\Gamma_{g_{1}}\left(u^{-}\right)_{1}-\Gamma_{g_{2}}\left(u^{-} 1\right)_{2}\right|_{N} \\
& =d[T(y)] \\
& \leq \sup _{y} d[\mathcal{T}(y)]=\mathcal{D}(\mathcal{I})
\end{aligned}
$$

where $d($.$) and \mathcal{D}(\mathcal{I})$ defines the radius and the diameter of information of the set $\mathcal{T}$, respectively. Additionally, $g_{1}$ and $g_{2}$ represent two systems within the consistency set. It should be noted here that the set $\mathcal{T}(y)$ forms a convex symmetric set with symmetric point $g_{s}=0$ and $\eta=0$, and there exists a function in $\mathcal{T}(y)$ that is compatible with the zero outcome: $y_{k}=0, \forall_{k} \in(1: N-1)$. The diameter of information can be defined by [76]

$$
\mathcal{D}(\mathcal{I}) \leq \sup _{g \in \mathcal{T}(0)}\left|p . i_{N}+\sum_{j=0}^{N} h_{N-j} . u_{j}+K . K_{u} \frac{\rho^{-N+1}}{\rho-1}\right|
$$


Consequently, the previous equation leads to

$$
\begin{aligned}
& \max \left|p . i_{N}+\sum_{j=0}^{N} h_{N-j} \cdot u_{j}+K \cdot K_{u} \frac{\rho^{-N+1}}{\rho-1}\right| \\
& \text { subject to } \\
& M(\mathbf{h})=\left[\begin{array}{cc}
K R^{-2} & \left(T_{h}^{N}\right)^{T} \\
T_{h}^{N} & K R^{2}
\end{array}\right] \geq 0 \\
& \left|y-\left(T_{u}^{N} p P+T_{u}^{N} h\right)-x\right| \leq \epsilon \\
& \left|x_{j}\right| \leq K . K_{u} \frac{\rho^{-j}}{\rho-1} \\
& |u(N)| \leq u_{\max } \\
& \left|E p o_{N}\right| \leq E p o_{\max } \\
& \left|h_{N}\right| \leq K \rho^{-N}
\end{aligned}
$$

Using Equation (5.16), the worst-case identification error can be computed to validate the model [75].

$$
\begin{array}{r}
x_{k}=p \cdot E P O_{k}+x_{k}^{n p} \\
\text { where } \\
\left|x_{n}^{n p}\right| \leq K \frac{\rho^{-n}}{\rho-1} K_{u} \\
x_{k}=p \cdot \sum_{j=-\infty}^{k}\left(E P O_{j}-E P O_{j-1}\right)=p \cdot E P O_{k}
\end{array}
$$

\subsection{ARX MODEL}

The autoregressive with exogenous input (ARX) modeling structure is a versatile linear parametric technique that is capable of estimating system models from measure- 
ment samples. This modeling structure is the most commonly used parametric system identification techniques due to its simplicity and ability to estimate complex dynamical models. The general structure of the ARX model is given in Equation (5.17) where $\frac{B(q)}{A(q)}$ is the system transfer function and $\frac{1}{A(q)}$ is the noise transfer function and $e(k)$ is assumed to be of white Gaussian form. The parameter $q$ is a forward-shift operator such as $q^{-1} u_{k}=u(k-1)$. In this structure, it is assumed that both the input signal, $u(k)$, and the output measurements, $y(k)$, are known and available. Using both the input and the output information, the aim is to estimate the system transfer function $\frac{B(q)}{A(q)}$. Due to its simplicity and versatility, the ARX is often referred to as "the mother of all" dynamical models [67]. As such, ARX model results are used as a benchmark against the semi-blind identification results. Table 5.3 provides a summary of results from the ARX model. It should be emphasized that although certain average response values are better with the ARX identification method, their maximum prediction error value are significantly higher than those obtained from the semi-blind identification techniques. This is because the semi-blind robust identification method attempts to minimize the maximum possible error that can be attained while the classical identification method attempts to minimize the average predication error.

$$
y(k)=\frac{B(q)}{A(q)} u(k)+\frac{1}{A(q)} e(k)
$$




\begin{tabular}{|l|c|c|}
\hline Patient\# & error $_{\max }$ & error $_{r m s}$ \\
\hline 1 & 2.68 & 0.40 \\
28 & 2.28 & 0.31 \\
30 & 5.74 & 0.84 \\
6 & 4.22 & 0.67 \\
37 & 2.65 & 0.33 \\
\hline
\end{tabular}

TABLE 5.3

Average and maximum prediction errors from ARX results.
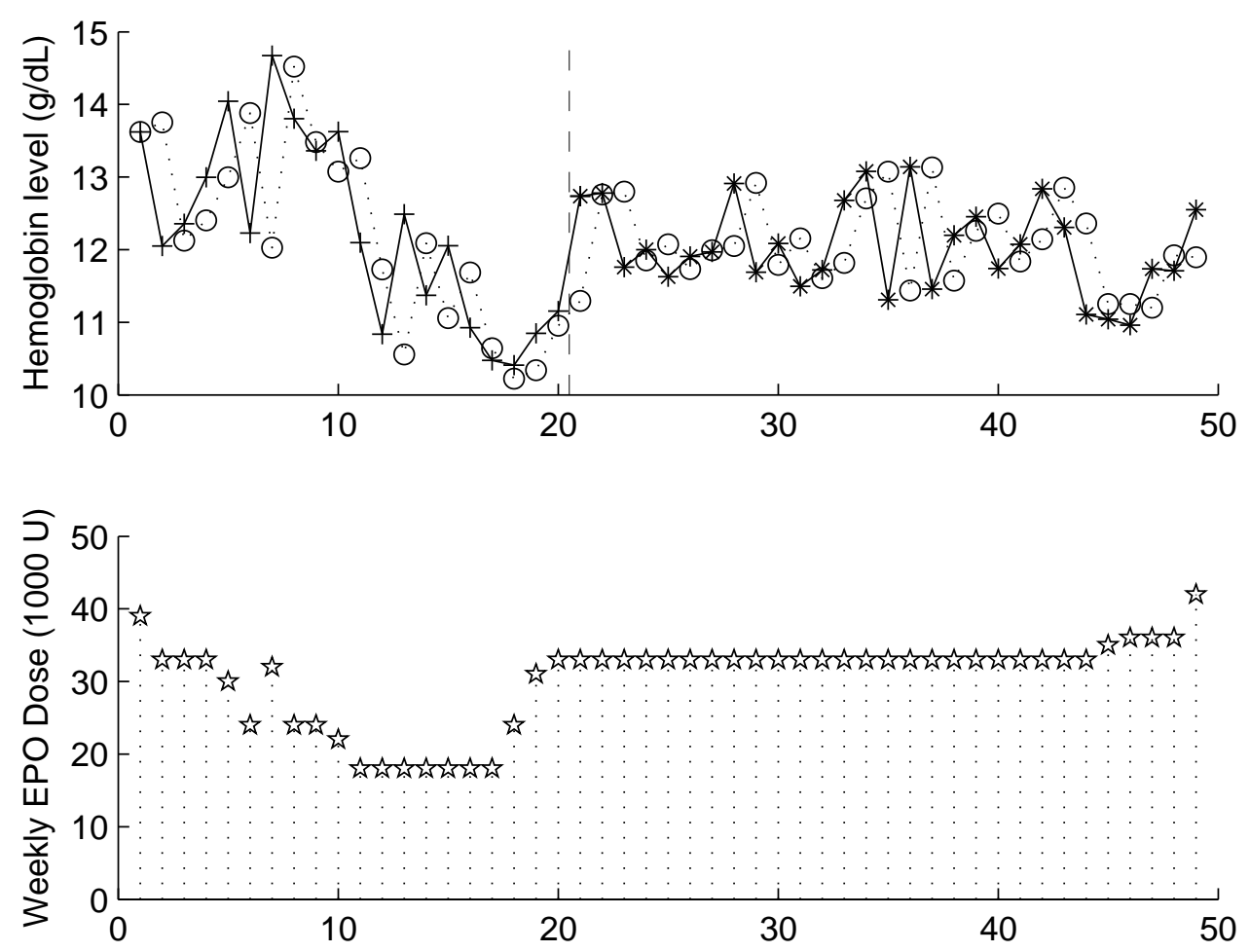

FIGURE 5.9-ARX model results for patient \#37. [Top] Hb prediction with a vertical line used to demarcate data used for identification and for forecasting. A (+) is used to denote experimental data used during the identification and (*) is used to denote data not used in the identification. (o) symbol is used to indicate predicted results with $1^{\text {st }}$ order model. [Bottom] Administered erythropoietin dose in x1000 Units per week. 

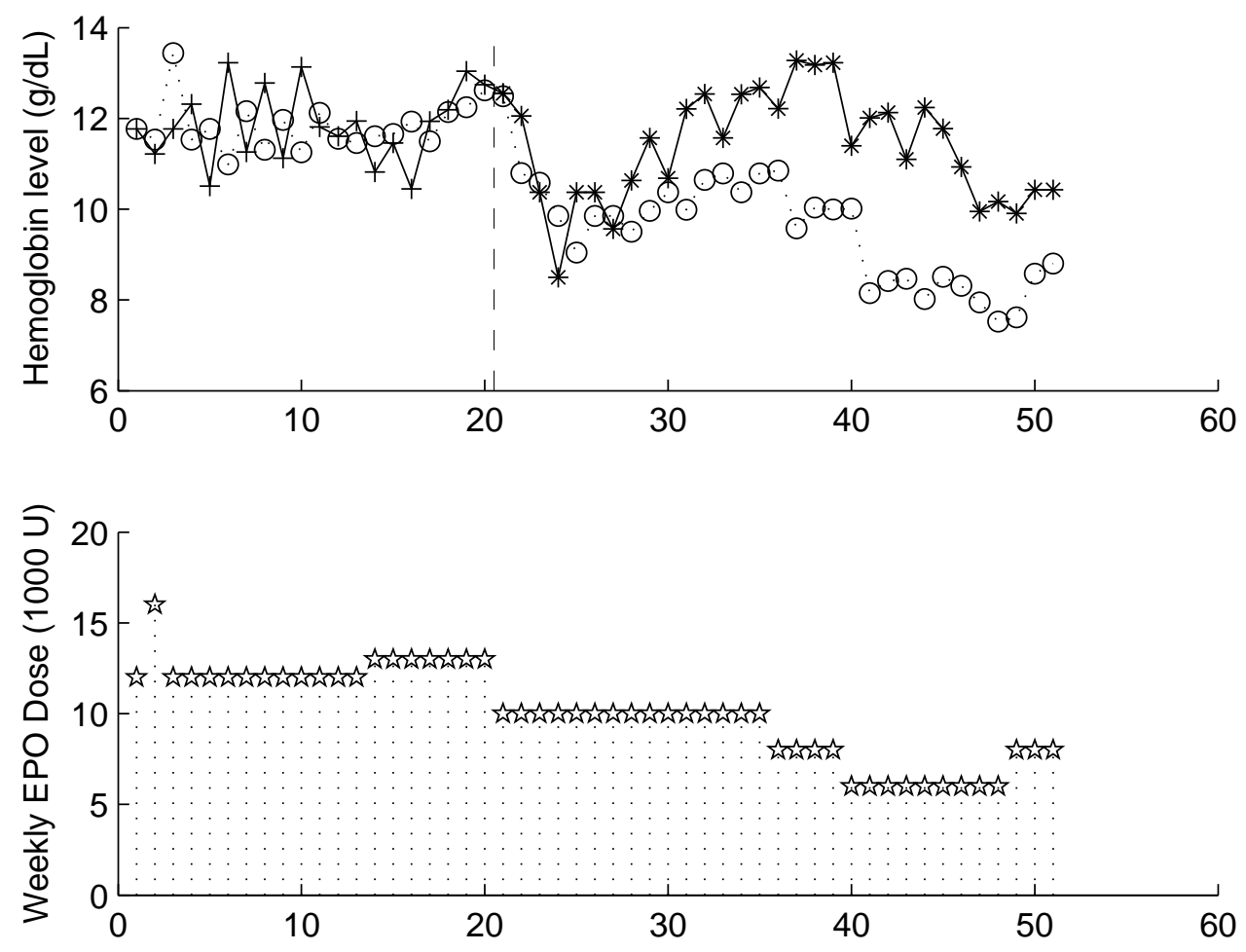

FIGURE 5.10-ARX model results for patient \#6. [Top] Hb prediction with a vertical line used to demarcate data used for identification and for forecasting. A (+) is used to denote experimental data used during the identification and $(*)$ is used to denote data not used in the identification. (o) symbol is used to indicate predicted results with $2^{\text {nd }}$ order model. [Bottom] Administered erythropoietin dose in x1000 Units per week. 

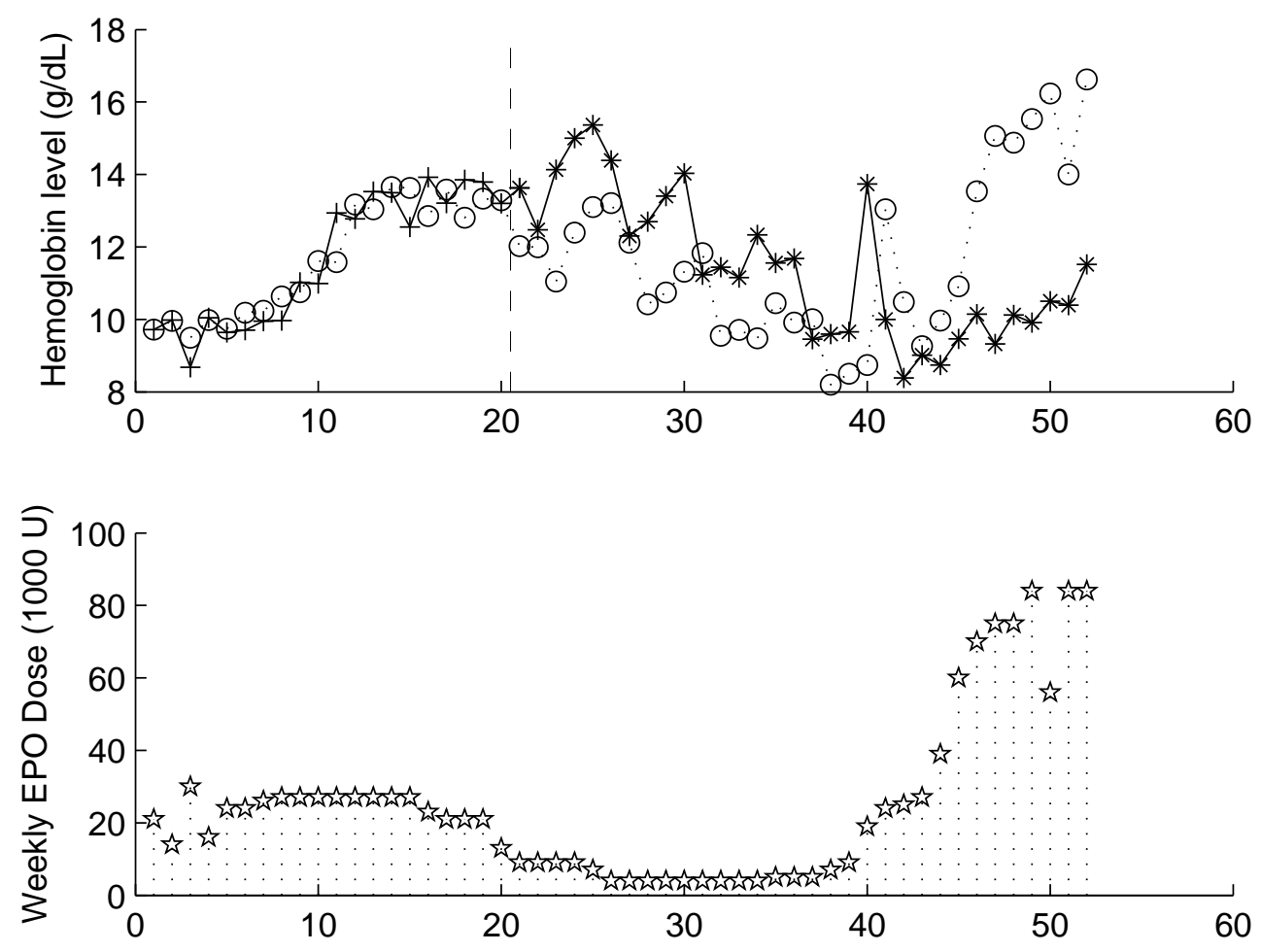

FIGURE 5.11 - ARX model results for patient \#30. [Top] Hb prediction with a vertical line used to demarcate data used for identification and for forecasting. A (+) is used to denote experimental data used during the identification and $(*)$ is used to denote data not used in the identification. (o) symbol is used to indicate predicted results with $1^{\text {st }}$ order model. [Bottom] Administered erythropoietin dose in x1000 Units per week. 

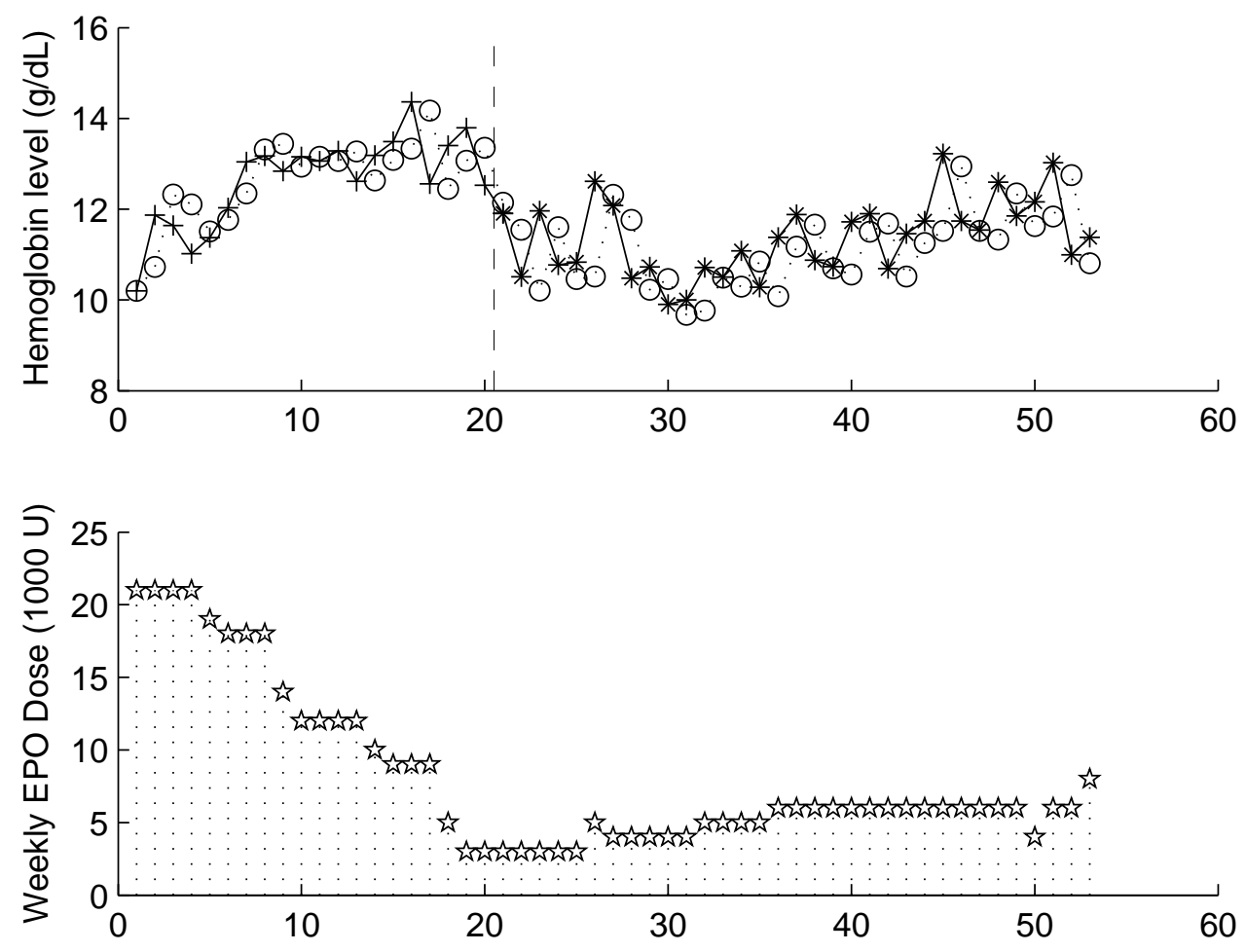

FIGURE 5.12 - ARX model results for patient \#28. [Top] Hb prediction with a vertical line used to demarcate data used for identification and for forecasting. A (+) is used to denote experimental data used during the identification and $(*)$ is used to denote data not used in the identification. (o) symbol is used to indicate predicted results with $2^{\text {nd }}$ order model. [Bottom] Administered erythropoietin dose in x1000 Units per week. 

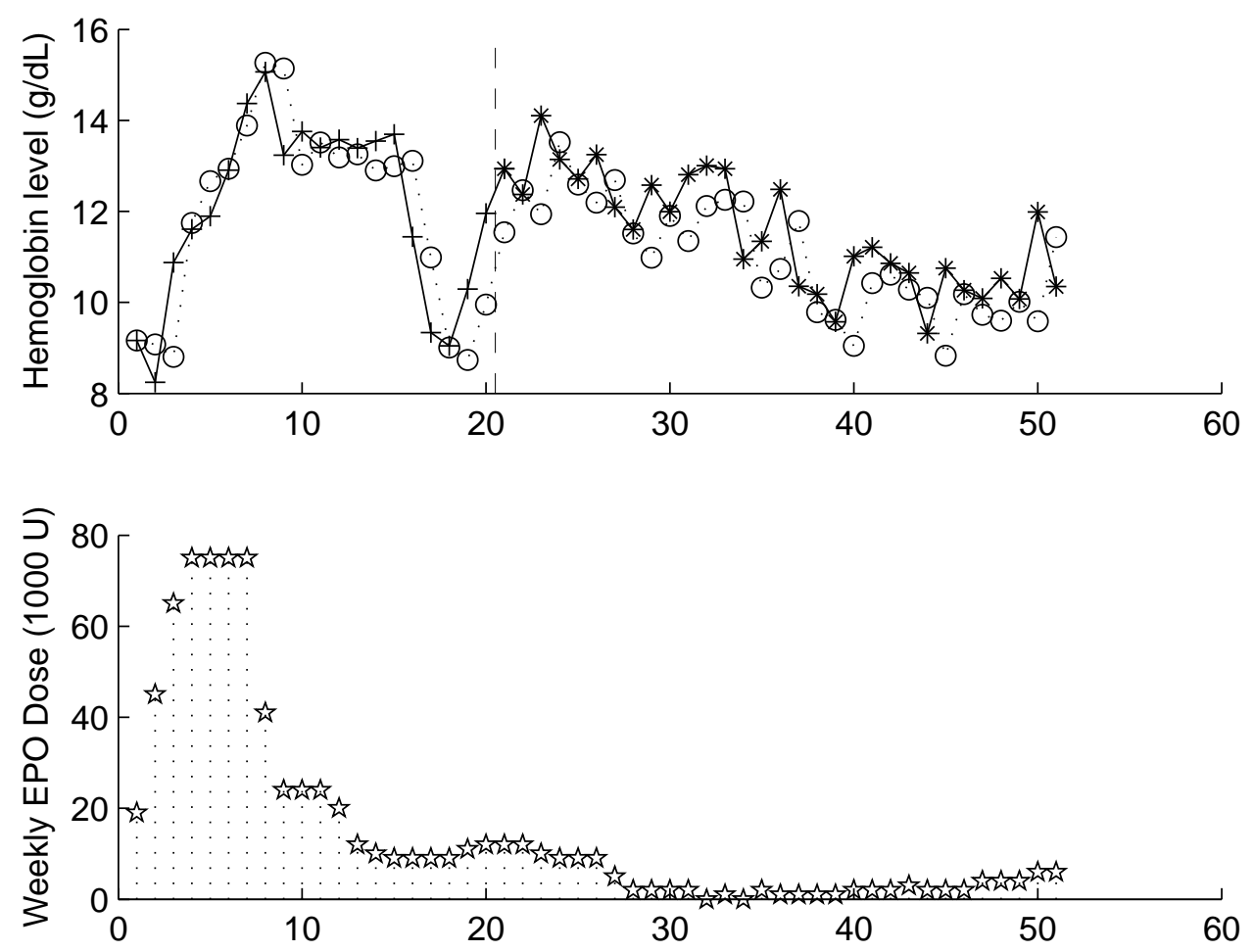

FIGURE 5.13 - ARX model results for patient \#1. [Top] Hb prediction with a vertical line used to demarcate data used for identification and for forecasting. A (+) is used to denote experimental data used during the identification and $(*)$ is used to denote data not used in the identification. (o) symbol is used to indicate predicted results with $3^{\text {rd }}$ order model. [Bottom] Administered erythropoietin dose in x1000 Units per week. 


\section{CHAPTER 6 \\ ROBUST CONTROL SYNTHESIS FOR ANEMIA MANAGEMENT PROBLEM}

The previous chapter provided techniques that derives mathematical models from measurement data using semi-blind robust identification. This chapter extends on that development by designing robust feedback controller in the $\mu / H_{\infty}$ design framework from the derived models. Additionally, a robust control design technique is provided to strategize dosage regimen for newly admitted patients using a second order transfer function equation that represents RBC profile.

\subsection{Robust CONTROL SYNTHESIS}

Consider the feedback control configuration in Figure 6.1 with the plant defined by $G$, reference signal $r$, measured signal $y$, and a disturbance signal $d$. The design objective is to derive a controller $K$ such that the measured signal $y$ mimics the behavior of a certain reference signal $r$, despite the presence of unknown disturbance $d$. In classical control design problems, this objective is achieved while assuming the mathematical description of the process $G$ accurately represents the true system to be controlled. However, it is well understood that system $G$ is only an approximation of the actual system as certain dynamics are often excluded at the model derivation stage in order to achieve tractable results. As such, it is importation to describe system $G$ as a family of models consisting of a nominal model and uncertainty in the model. Then, the robust control 


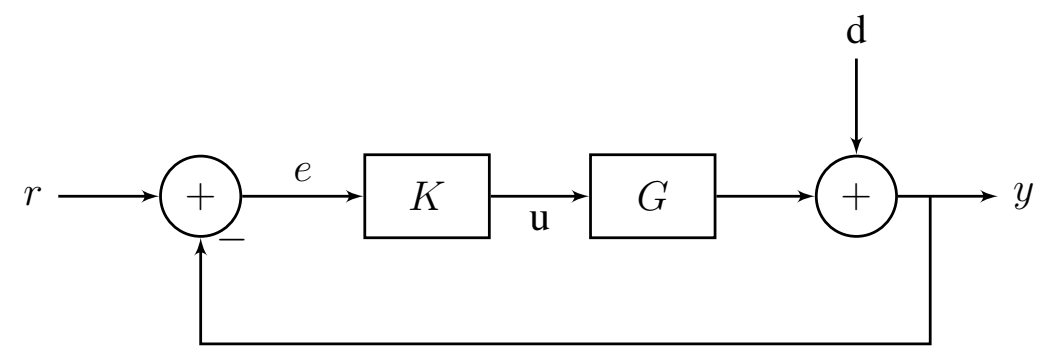

FIGURE 6.1: Feedback system.

design problem becomes designing a single controller $K$ such that the set of plants described by the family of models satisfies the required performance specifications.

Several uncertainty model descriptions are available in the literature [83]; however, the two commonly used descriptions are the additive and multiplicative uncertainty. In this thesis, the multiplicative model uncertainty considered as this description directly provides the means to describe uncertainty as a percentage change in the nominal value over the frequency of interest. Equation (6.1) describes this multiplicative uncertainty where $G_{p}(s)$ is the generalized plant, $G(s)$ defines the nominal model, and $W_{m}(s)$ is an unknown function that bounds the generalized system plant as shown in Equation (6.2). Figure 6.2 shows the feedback configuration with the multiplicative uncertainty description.

$$
\begin{array}{r}
G_{p}(s)=\left[1+W_{m}(s) \Delta(s)\right] G(s) \\
|\Delta(j \omega)| \leq 1, \quad \forall_{\omega} \\
M(\omega)=\max _{G_{p} \in \mathcal{G}}\left|\frac{G_{p}(j \omega)-G(j \omega)}{G(j \omega)}\right| \\
\left|W_{m}(j \omega)\right| \geq M(\omega) \quad \forall_{\omega}
\end{array}
$$




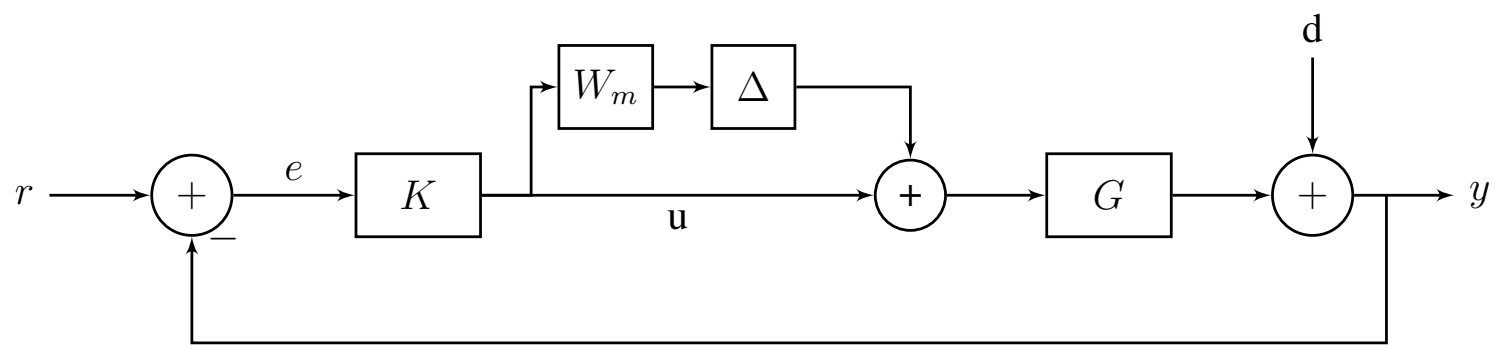

FIGURE 6.2: Multiplicative uncertainty in a feedback loop.

\subsection{Robust Controller Design: Some Definitions}

In feedback control design problems, a controller is considered to be robust if the following definitions are satisfied:

\subsubsection{Nominal Stability (NS)}

Nominal stability is the basic requirement of any feedback control system. The Nyquist stability criterion can be used to test for nominal stability conditions while assuming zero uncertainty in the model. That is by making $\Delta=0$.

\subsubsection{Nominal Performance (NP)}

The nominal performance condition ensures that the designed controller satisfies performance specifications. This can be achieved by solving for the closed loop transfer function of Figure 6.1, for example. From the figure, the closed loop transfer function is defined in Equation (6.3) where $G$ is the nominal system and $K$ is the controller to be designed.

$$
T_{y r}=\frac{G K}{1+G K}
$$

For tracking problems, it is generally required that the sensitivity function $S$, defined as $I-T_{y r}$, to be kept small in the frequency of interest. 


\subsubsection{Robust Stability (RS)}

The robust stability condition requires that the controller $K$ stabilizes the set of perturbed systems described in Equation (6.1), which includes the nominal system. In other words, given a controller $K$ that satisfies both NS and NP conditions, does the said controller also satisfy plants in the given set: $G_{0} \in G_{p} \subset \mathcal{G}$ ? If so, under what condition will the said controller not be valid? To answer the RS problem, consider the controller $K$ that stabilizes the system $G$ in Equation (6.1), it is shown that the perturbed complementary sensitivity function, defined in Equation (6.4) where $G_{i}$ is any system in the set $\mathcal{G}$, and $T_{y r}^{i}$ is its corresponding complementary function, is also stable provided Equation (6.5) is satisfied [83]. In the equation, $W_{m}$ is the multiplicative uncertainty function.

$$
\begin{gathered}
T_{y r}^{i}=\frac{G_{i} K}{1+G_{i} K} \\
\sup _{\omega}\left|T_{y r} W_{m}(j \omega)\right|<1, \quad \forall \omega \geq 0
\end{gathered}
$$

\subsubsection{Robust Performance (RP)}

The final objective of robust control design is to achieve the performance requirements for all systems in the set $\mathcal{G}$. Assuming the primary objective is to track a certain reference signal, as considered in the nominal performance case. Then, the controller $K$ achieves robust performance if Equation (6.6) is satisfied, where $S^{i}$ is the sensitivity function of all systems in $\mathcal{G}$

$$
\sup _{\omega}\left|S^{i}(j \omega) W_{m}(j \omega)\right|<1, \forall \omega \geq 0 .
$$

Equation (6.6) is shown to be equivalent to Equation (6.7) where $W_{d}$ is the disturbance weighting function.

$$
\left\|W_{d}(j \omega) S(j \omega)|+| W_{m}(j \omega) T(j \omega)\right\|_{\infty} \leq 1
$$




\subsection{Robust Control Design Solution to the Anemia Man- AGEMENT PROBLEM}

Results from the semi-blind robust identification models derived in Section 5.1 provided a relationship between erythropoietin (EPO) and hemoglobin $(\mathrm{Hb})$ measure of anemia patients under uncertainty. From these established results, the robust feedback controller be synthesized to optimize anemia management problems. For the purpose of the controller design, Equation (5.2) through Equation (5.6) are considered as the nominal models of the selected patients (Equation (5.2) is restated in Equation (6.8) as a reference). In each of these equations, the $H b(z)$ represents measured hemoglobin value while $E P O(z)$ represents dosage erythropoietin. From Equations (6.1) and (6.2), it is clear that the robust control synthesis is formulated in the continuous frequency domain while the derived transfer function of Equation (6.8) is of discrete time nature. However, by using the bilinear transformation [84], one can translate the discrete time Equation (6.8) into the corresponding continuous time of Equation (6.9). Similar transformation is applied to the other patient models. It should also be stated that to distinguish between other patient models, superscripts are used to indicate patient ID numbers. For example in Equations (6.8) and (6.9), superscript 1 represents patient \#1. However, to simplify the analysis, this identifier is omitted in the subsequent sections of this thesis, unless such an indicator is absolutely necessary.

$$
\begin{aligned}
& G^{1}(z)=\frac{H b(z)}{E P O(z)}=\frac{1.030 z^{3}+1.398 z^{2}+0.841 z-0.041}{z^{3}+0.317 z^{2}-0.501 z-0.816} \\
& G^{1}(s)=\frac{H b(s)}{E P O(s)}=\frac{0.515 s^{3}+1.459 s^{2} 15.11 s+25.88}{s^{3}+1.475 s^{2}+25.11 s}
\end{aligned}
$$




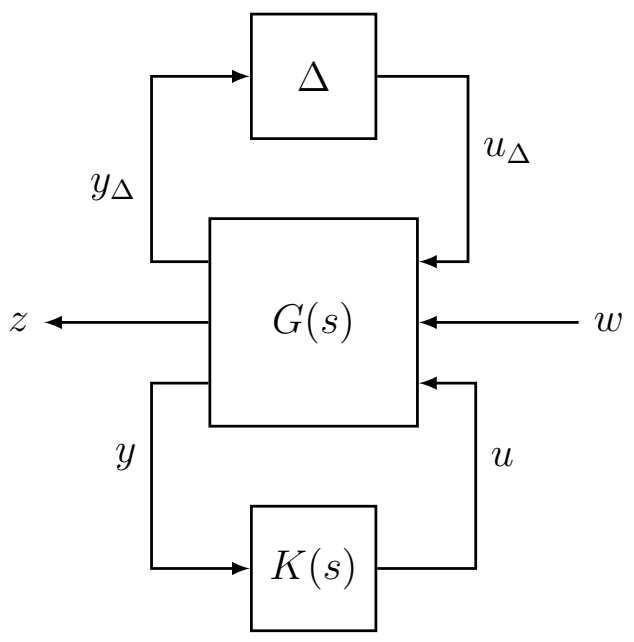

FIGURE 6.3-Generalized Plant Framework

\subsubsection{Generalized Plant Description}

The robust feedback control configuration shown in Figure 6.2 can be rearranged to represent Figure 6.3. In this configuration, $w$ represents all external inputs, including reference signal to the plant, $u$ represents the control inputs, $z$ represents output signal of interest including error signals, and $y$ represents the measured output which is the input to the controller $K$. In this setting, the overall objective is to determine a single controller $K$ that minimizes the output signal $z$. Equation (6.10) provides a summary of such dynamics.

$$
\begin{aligned}
\left(\begin{array}{l}
z \\
y
\end{array}\right) & =\left(\begin{array}{ll}
G_{11} & G_{12} \\
G_{21} & G_{22}
\end{array}\right)\left(\begin{array}{l}
w \\
u
\end{array}\right) \\
u & =K y
\end{aligned}
$$




\subsection{Weighting Functions}

In robust feedback control design methods, closed loop performance specifications are defined through opened loop weighting functions that can be translated into an optimization problem and then solved. The two most important objectives of the anemia control problem are that (1) the designed controller must track certain reference (desired) hemoglobin value and (2) when possible, dosage medication must be mini-

mized. Achieving these two objectives requires that both the error and dosage weighting functions to be included in the robust optimization process. In Figure 6.4, the weighting functions $W_{e}$ and $W_{u}$ are defined as an attempt to minimize both the error signal and dosage medication, respectively. Although there exist no direct method in determining these weighting functions, Equation (6.11) and Equation (6.12) are generally an excellent starting points. Parameters $M, w_{b}$, and $A$ in Equation (6.11) represents the percentage overshot, bandwidth, and steady state error, respectively. In Equation (6.12), $U_{\text {max }}$ defines the desired maximum input.

$$
\begin{gathered}
W_{e}=\frac{\frac{s}{M}+w_{b}}{s+w_{b} A} \\
W_{u}=\frac{1}{U_{\max }}
\end{gathered}
$$

\subsection{Augmented Plant}

The $\mathrm{H}_{\infty}$ controller design is a two stage control problem. First, the plant is augmented by removing the controller from the entire feedback loop, then the controller $K$ is determined to satisfy the system requirements. Figure 6.5 shows the augmented plant 


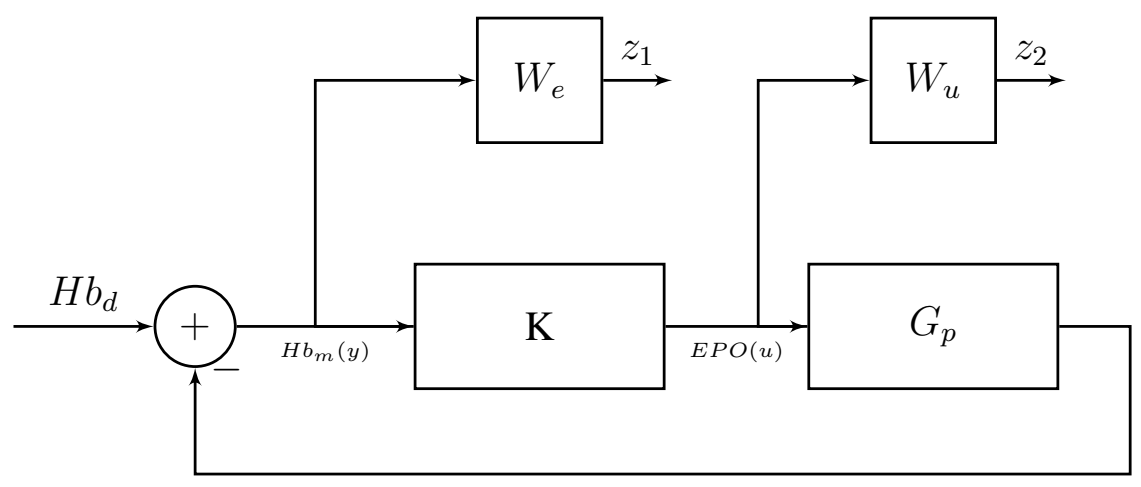

FIGURE 6.4: Anemia management as a robust control problem in a feedback loop.

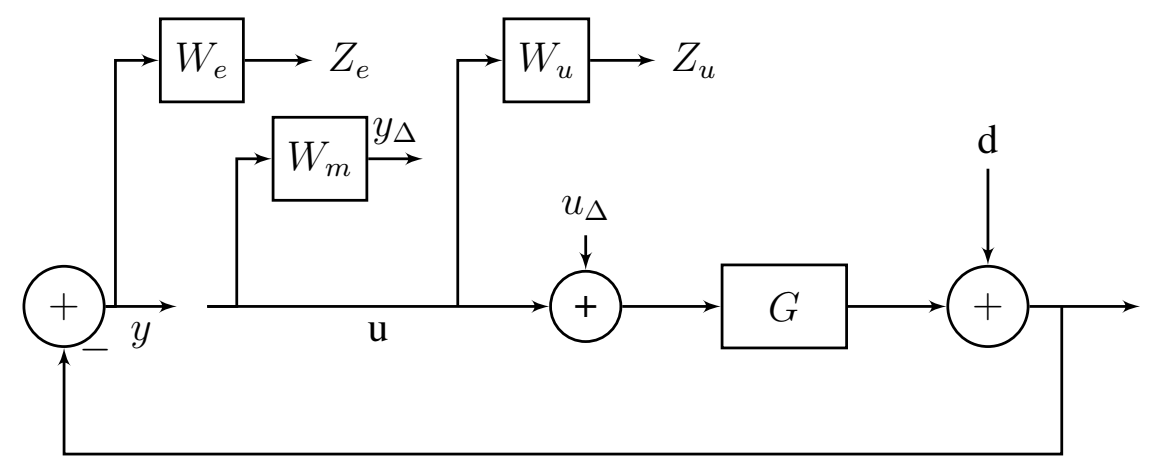

FIGURE 6.5: Uncertainty feedback system loop.

configuration where the controller is removed from the feedback loop. As previously defined, $W_{e}, W_{u}$, and $W_{m}$ are the system weighting functions representing the error, input, and the multiplicative uncertainty, respectively. From the figure, the augmented system matrix is derived and shown in Equation (6.13).

$$
\left[\begin{array}{l}
Z_{e} \\
Z_{u} \\
y_{\Delta} \\
y
\end{array}\right]=\left[\begin{array}{ccc}
-W_{e} G & -W_{e} & -W_{e} G \\
0 & 0 & W_{u} \\
0 & 0 & W_{m} \\
G & 1 & G
\end{array}\right]\left[\begin{array}{l}
u_{\Delta} \\
d \\
u
\end{array}\right]
$$




\section{$6.6 \mathrm{H}_{\infty}$-CONTROL SYNTHESIS AND RESULTS}

The feedback system in Figure 6.4 represents the overall performance objective of the robust control design. In the figure, the uncertainty system $G_{p}$ defines a set containing perturbed plants as defined in Equation (6.2). As previously stated, the objective is to design a controller that satisfies certain performance specification. To achieve this objective via robust feedback control setup, weighting functions are introduced to the feedback dynamics. As an example, Equations (6.14) through (6.16) show the weighting functions used to design robust controller for patient \#1. They represent the error, multiplicative, and control input weighting functions, respectively. Figure 6.6 and 6.7 show simulated results of patient \#1 and \#37. In each of these figures, the top portion represents output $\mathrm{Hb}$ response values while the bottom shows the input EPO administration. Additionally, the four plots in each of these figures represent four possible patient Hb profiles.

$$
\begin{gathered}
W_{e}^{1}=\frac{0.8333 s+0.03}{s+0.00282} \\
W_{m}^{1}=\frac{s+0.1}{s+1} \\
W_{u}^{1}=\frac{1}{4000}
\end{gathered}
$$



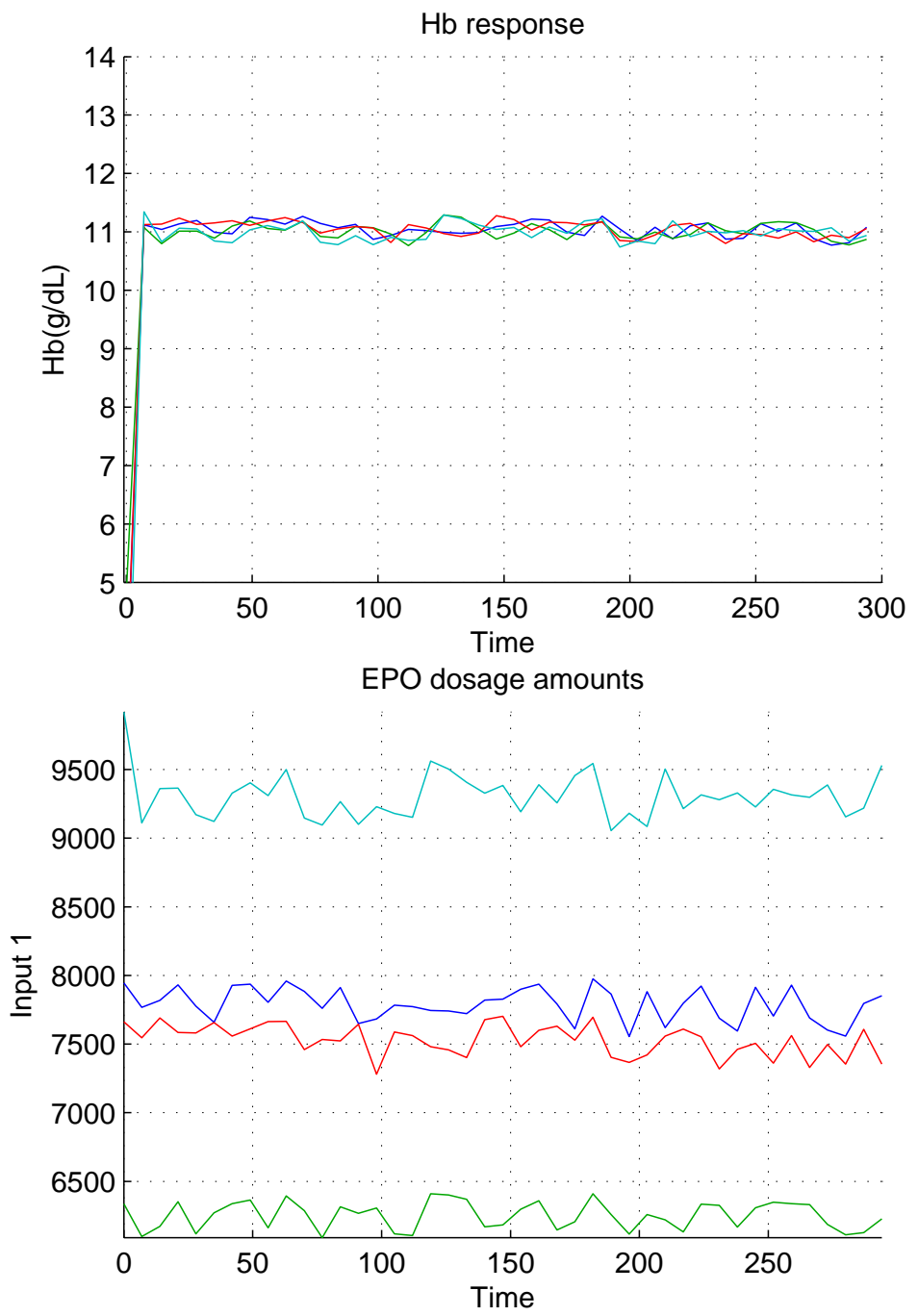

FIGURE 6.6 - Robust control results for patient \#1. [Top] Hemoglobin response. [Bottom:] EPO values. 

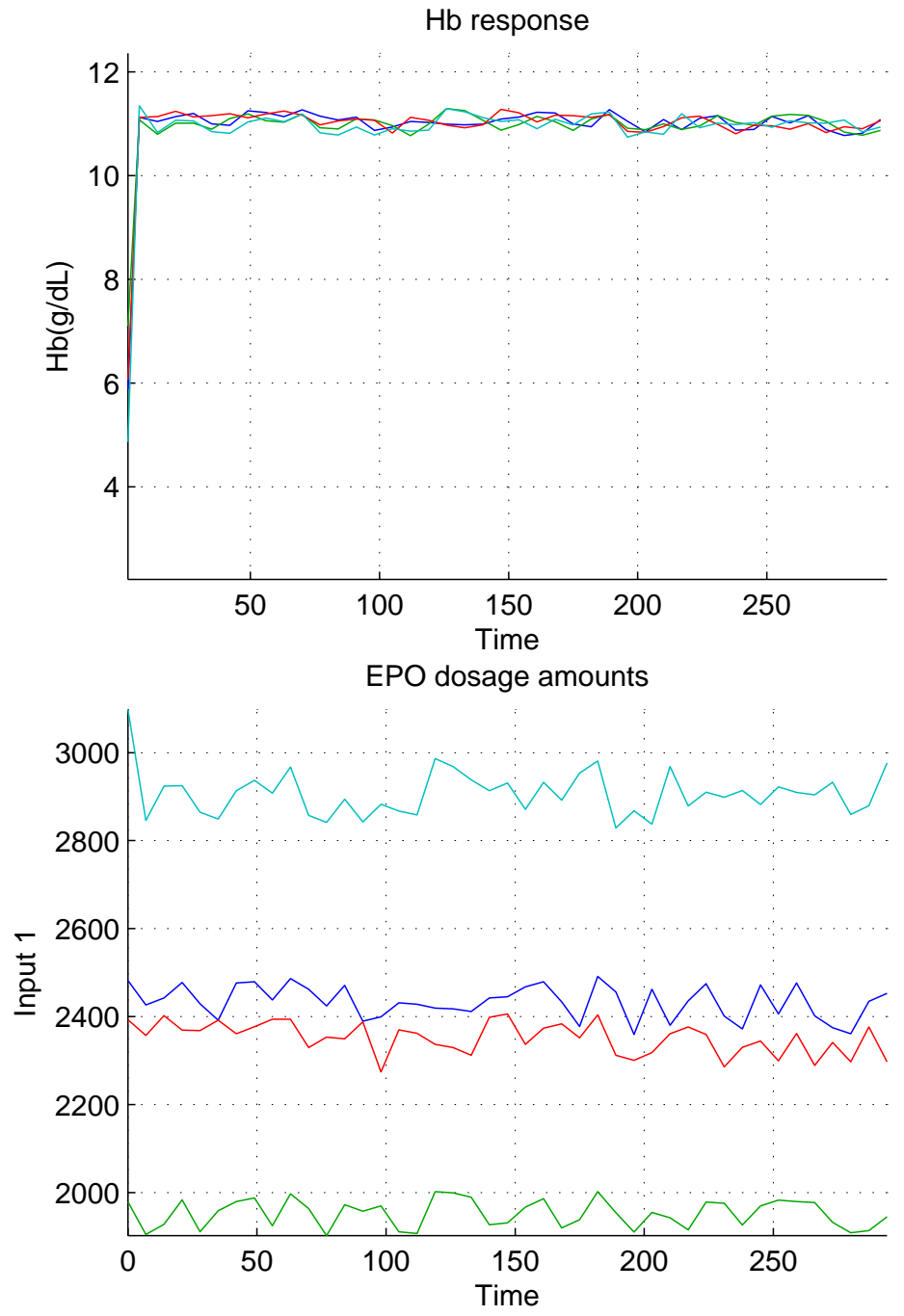

FIGURE 6.7 - Robust control results for patient \#37. [Top] Hemoglobin response. [Bottom:] EPO values. 


\subsection{Robust Control Design for the Initial Dose Regimen}

To provide personalized model using the semi-blind robust identification technique introduced in the previous chapter requires that at least fifteen measurement samples to be available. Since $\mathrm{Hb}$ values are measured on a weekly basis, this requirement translates into fifteen weeks of data and since this time frame is too long for patients to receive treatment, a procedure is provided to provide initial dosage regimen from the robust control design framework. This is achieved by introducing a second order transfer function equation with unknown parameter values $k$ and $\tau$ as shown in (6.17). In this equation, the constant $k$ defines sensitivity to the medication. This value ranges from $0.1-0.9$ and it indicates responsiveness to the medication. A low value shows insensitive to the medication while high $k$ value shows very responsive to medication. The $\tau$ parameter value defines red blood cell (RBC) lifespan that ranges from $60-120$ days.

$$
G_{\text {initial }}=\frac{k}{(s \tau+1)^{2}}
$$

\subsubsection{Baseline Hb Level}

In addition to the $k$ and $\tau$ parameters of Equation (6.17), baseline hemoglobin value must also be specified. This value defines patient's initial $\mathrm{Hb}$ value prior to treatment and it ranges from $6-8 \mathrm{~g} / \mathrm{dL}$. This is defined here as $H b_{0}$. As previously stated,

the objective of the feedback controller design is to increase patient's hemoglobin value from $H b_{0}$ to the desired range of $10-12 \mathrm{~g} / \mathrm{dL}$. From the robust control design point of view, this parameter value introduces an additional uncertainty in the robust controller design process. Figure 6.8 shows the step response profile of the nine possible transfer functions for low, middle, and high values of $k$ and $\tau$ from Equation (6.17) with the nominal $H b_{0}$ of 7 to represent the starting hemoglobin value of a hypothetical patient. Table 6.1 provides a summary of $k$ and $\tau$ values where the midpoint values are used for 


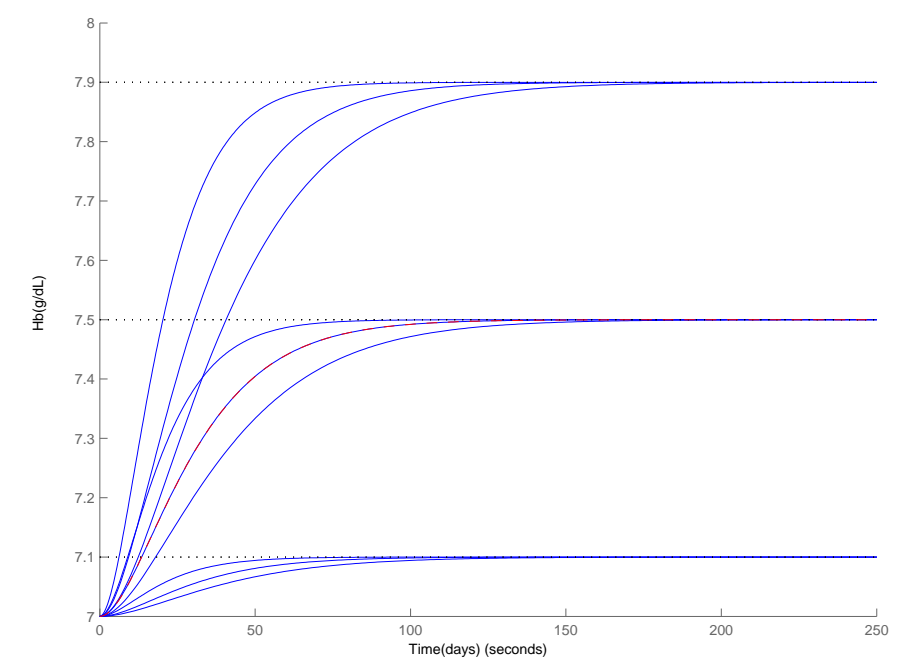

FIGURE 6.8-Step response of partitioned system plant

\begin{tabular}{|c|c|c|}
\hline & $k$ & $\tau$ \\
\hline Low & 0.1 & 60 \\
Mid & 0.55 & 90 \\
High & 0.9 & 120 \\
\hline
\end{tabular}

TABLE 6.1

Low, Mid, and High values of $k$ and $\tau$ parameters.

the nominal transfer function indicated by red in Figure 6.8. As can be observed in the figure, a baseline hemoglobin, $H b_{0}$, value of $7 \mathrm{~g} / d L$ is selected as a the nominal baseline hemoglobin.

\subsubsection{Weighting Function}

The weighting function as defined in the previous section is a mathematical equation that is used to encompass all possible plants in the robust controller design. Figure 6.9 shows the Bode plot of the uncertainty system with the multiplication uncertainty function shown in red which has the transfer function equation shown in Equation (6.18). 


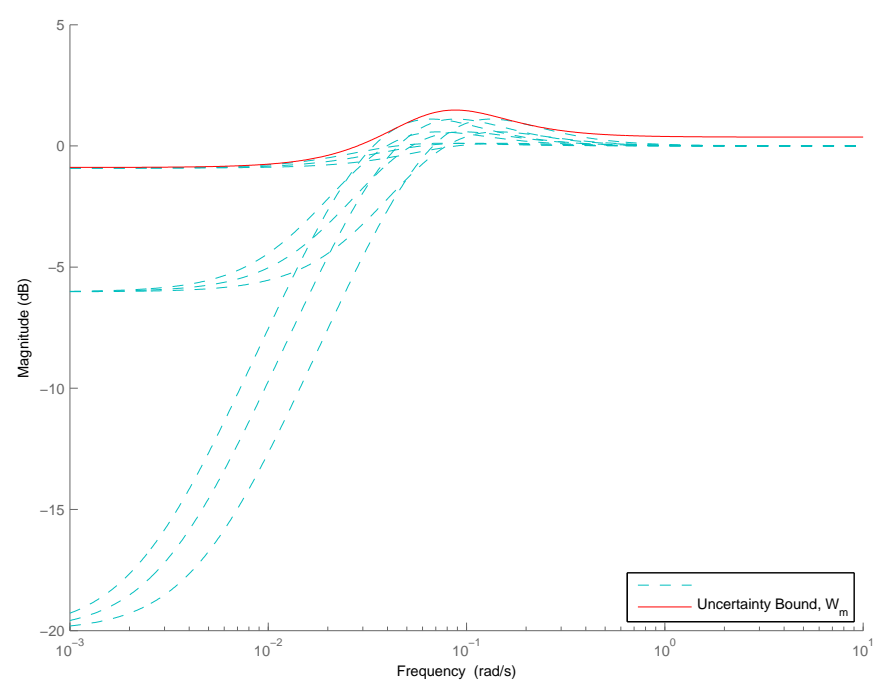

FIGURE 6.9-Bode plot of uncertainty function.

Figure 6.10 shows the corresponding step response of possible plant dynamics for the given parameter values.

$$
W_{m}^{a}=\frac{1.043 s^{2}+0.1553 s+0.00508}{s^{2}+0.1219 s+0.00566}
$$

\subsection{CONTROLler Design}

\subsubsection{Results}

Figure 6.11 shows the robust controller results of possible patients response profile from the system set defined by the transfer function Equation (6.17). In the figure, the top figure represents the output $\mathrm{Hb}$ value while the bottom one represents the input EPO medication. Each line could be considered as a possible patient response profile for a given $\mathrm{EPO}$ and $\mathrm{Hb}$. Also shown are three randomly selected baseline $\mathrm{Hb}$ values from the range $6-8 \mathrm{~g} / \mathrm{dL}$. It is clearly seen that the controller is able to stabilize the 


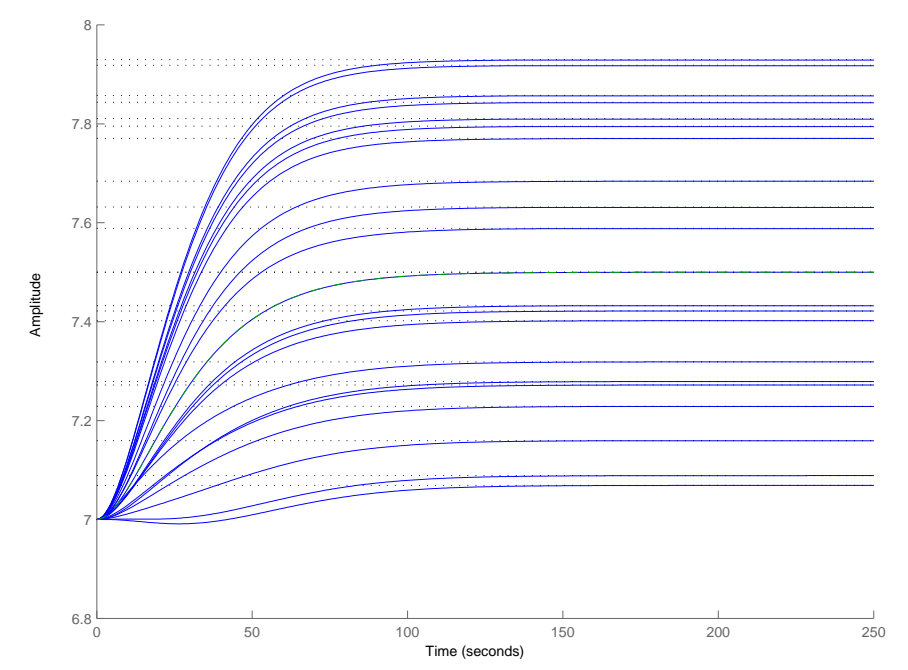

FIGURE 6.10 -Step response of possible plant dynamics.

patient response value to the desired $\mathrm{Hb}$ value of $11 \mathrm{~g} / \mathrm{dL}$. From the figure, however, one can observe two anomalies: 1.) the wide range of dosage medication ( approximately 3,000 to 120,000 Units), and 2.) the slow response profile for certain patient ( up to 1,500 days). These usual behavior is the controller's attempt to satisfy the design requirements for the wide range in parameters describing the transfer functions of the system set described in Equation (6.17). A solution to address these issues is to a robust controller for functions with a smaller parameter ranges that can be achieved by partitioning all parameter spaces of the transfer function in Equation (6.17).

\subsection{Parameter Space Partition and Results}

To design a control strategy to mimic response value of an actual patient, parameters of Equation (6.17), $k$ and $\tau$, must be partitioned to reflect poor, average, and high response. Table 6.2 shows the partitioned parameter space for both $k$ and $\tau$ values.

Figures $6.12,6.13$, and 6.14 show $H_{\infty}$ control simulation results for poor, av- 

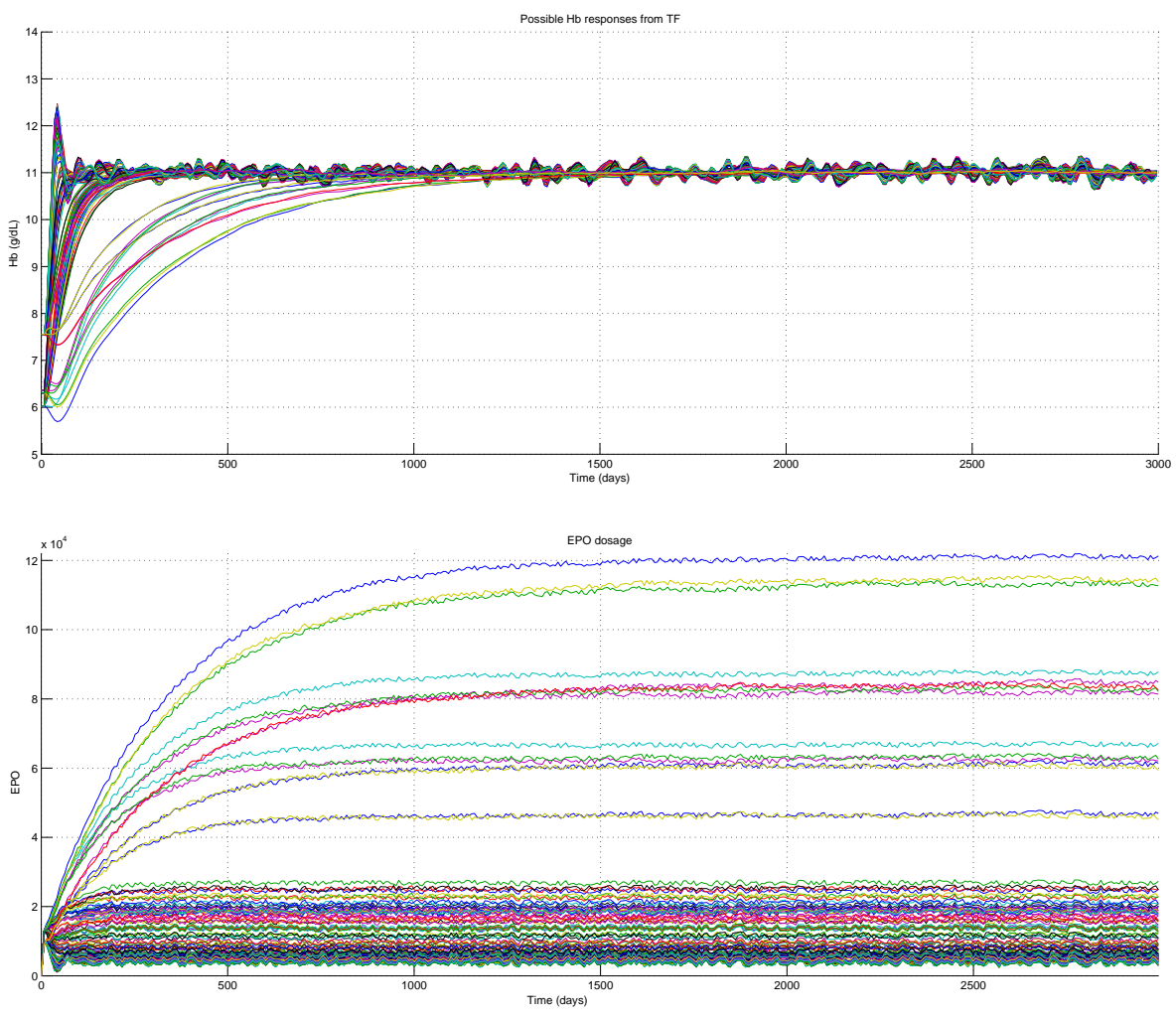

FIGURE 6.11-Controller result for the general transfer function equation. Top: $\mathrm{Hb}$ response stabilized at $11 \mathrm{~g} / \mathrm{dL}$. Bottom: EPO medication. 


\begin{tabular}{cc}
\hline$\tau$-range & $k$-range \\
\hline $60-80$ & $0.1-0.25$ \\
- & $0.4-0.65$ \\
- & $0.65-0.9$ \\
$80-100$ & $0.1-0.25$ \\
- & $0.4-0.65$ \\
- & $0.65-0.9$ \\
$100-120$ & $0.1-0.25$ \\
- & $0.4-0.65$ \\
- & $0.65-0.9$ \\
\hline
\end{tabular}

TABLE 6.2

Partitioned $k$ and $\tau$ parameter ranges.

erage, and highly responsive patients under EPO medication. It can be noted that by refining the parameter spaces to short ranges, better controller performance is achieved. In each of these figures, the four plot lines show two different patient response profile that starts from two randomly selected baseline $\mathrm{Hb}$ value. Their corresponding EPO profiles are shown at in the bottom figures. 

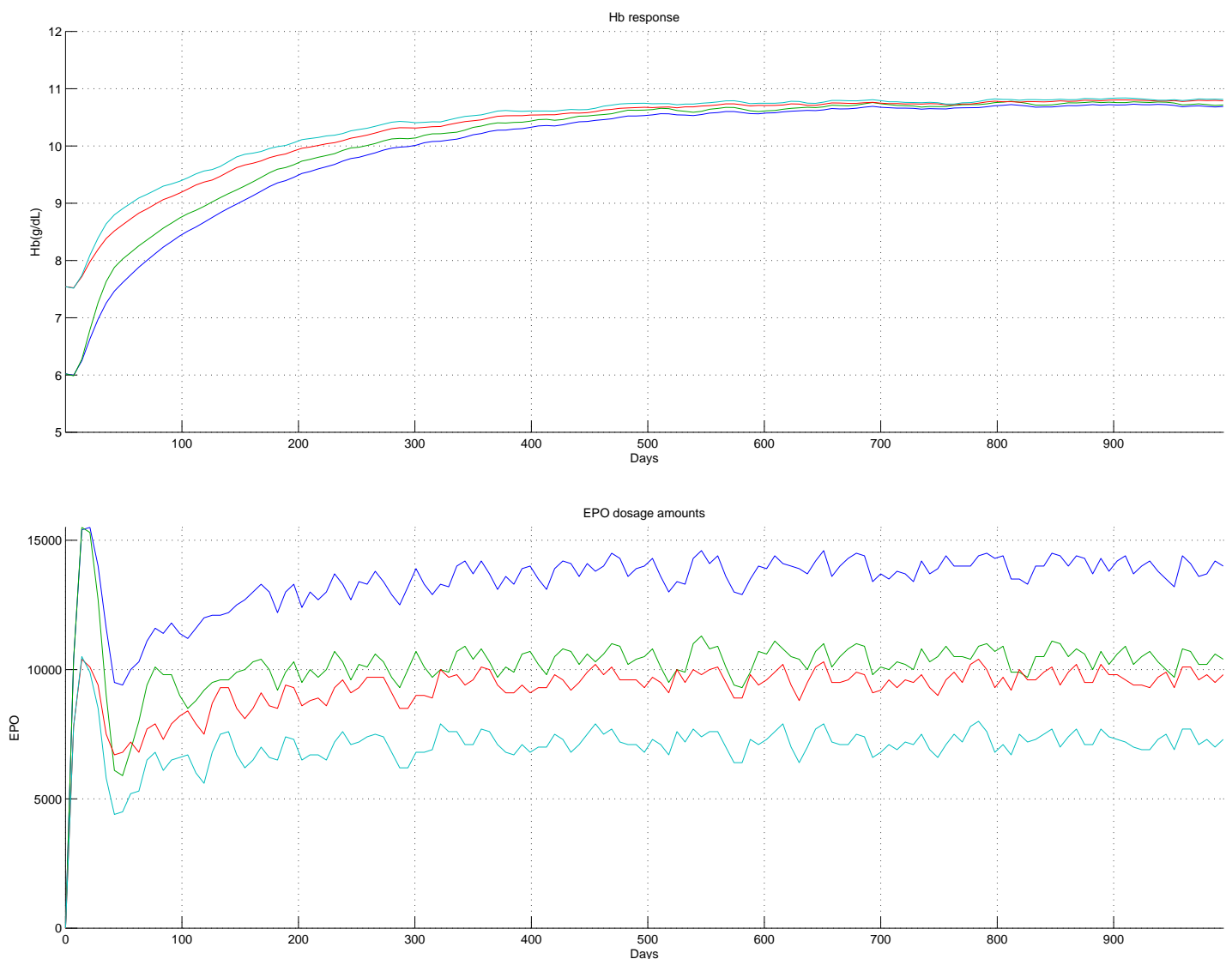

FIGURE 6.12-Hb (top) vs. EPO (bottom) for poorly responsive patients 

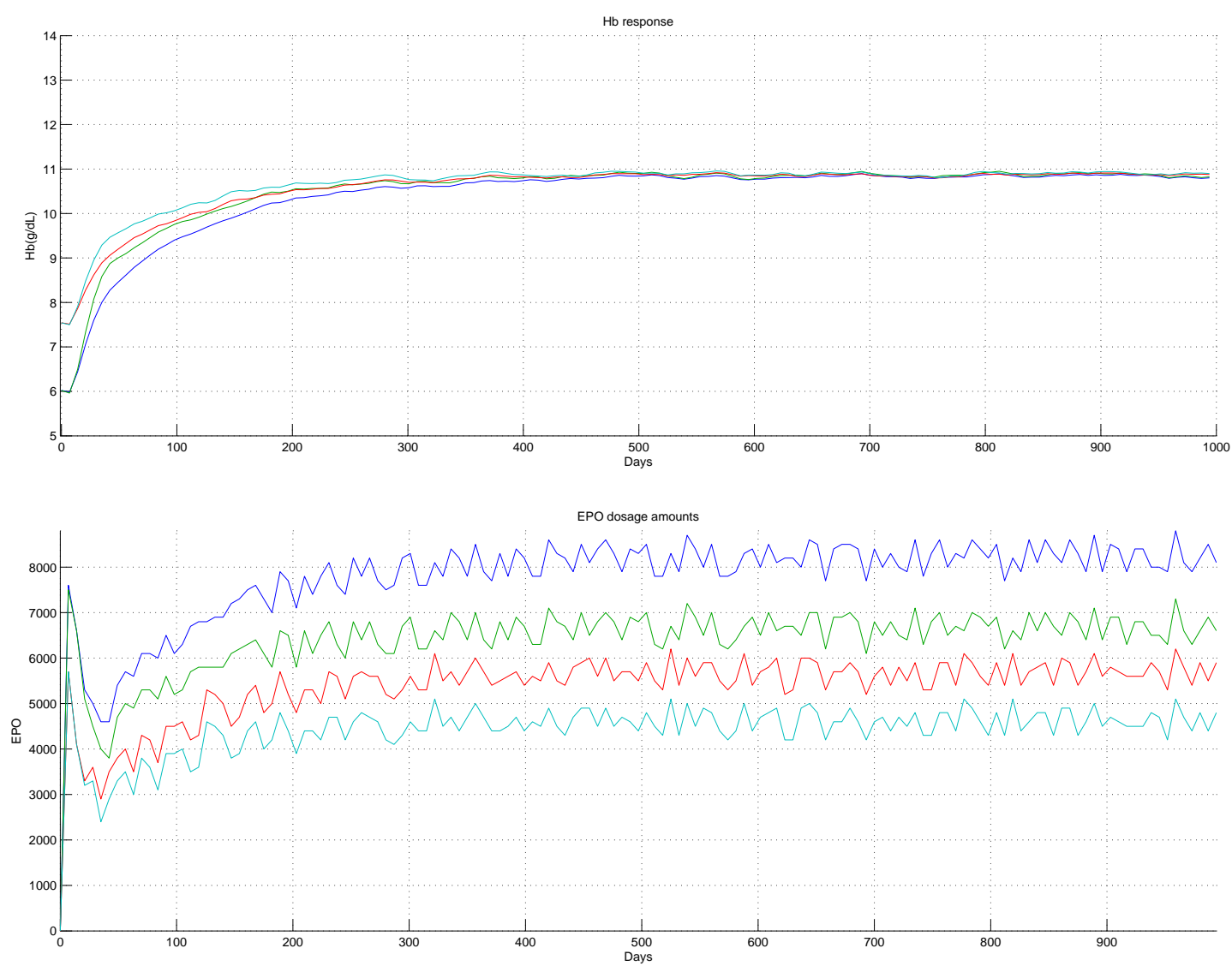

FIGURE 6.13 - Hb (top) vs. EPO (bottom) for average responsive patients 

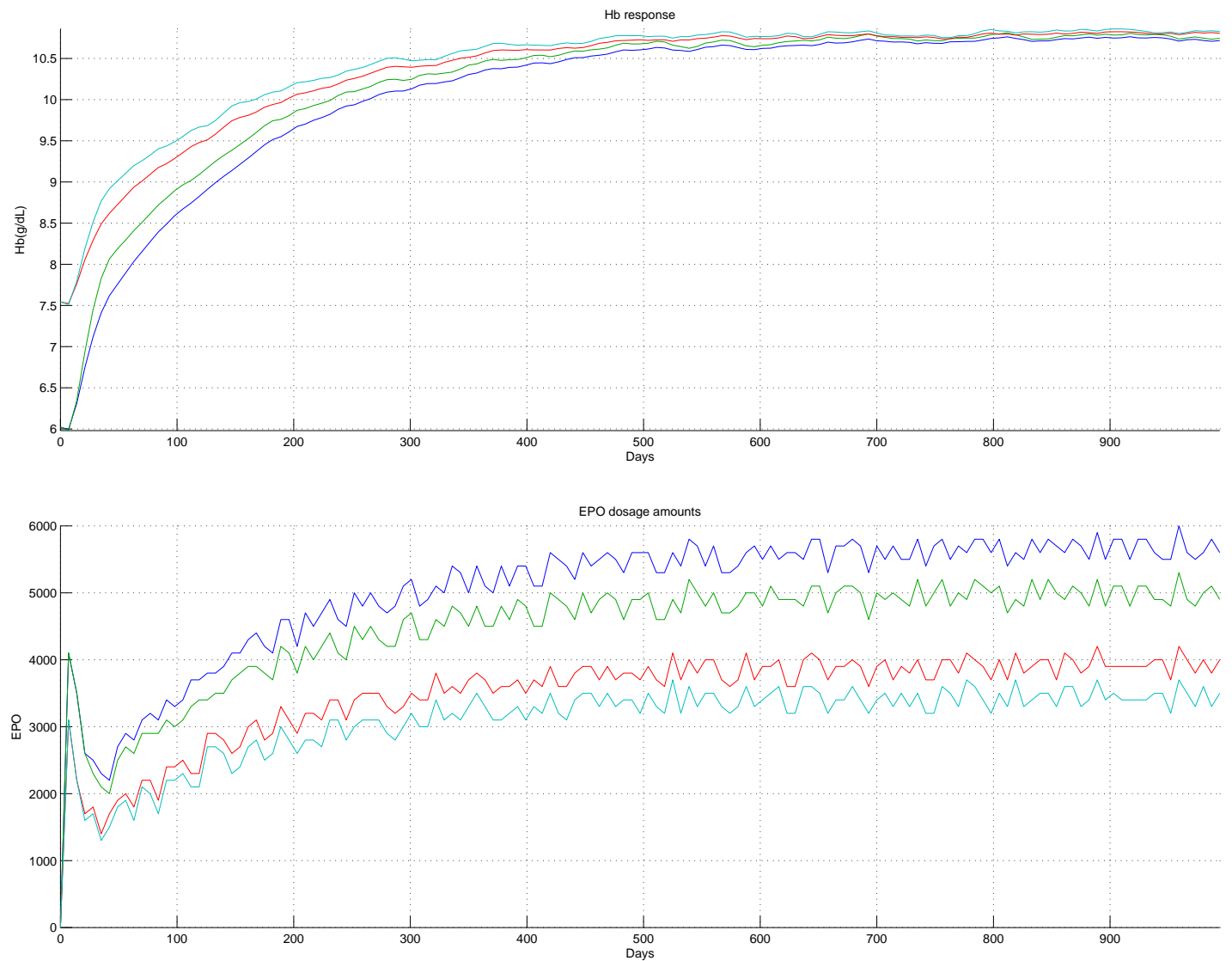

FIGURE 6.14- Hb (top) vs. EPO (bottom) for good responsive patients 


\section{CHAPTER 7 CONCLUSIONS AND FUTURE WORK}

\subsection{CONCLUSION}

Personalizing treatment regimen for patients with chronic kidney condition is a major challenge to clinicians. This is primarily due to the fact that most of the existing approaches focus on addressing the issue as a population-wide problem that rely on the performance of the general patient population responses to the drug treatment. However, the presence of inter-variability in erythropoietic responses makes such a dosing strategy nonoptimal with respect to the cost of the medication. In some instances, this strategy may be potentially dangerous to the welfare of certain patients. An alternative approach is to individualize treatment strategy by developing models based uniquely on the individual patient's hemoglobin response to erythropoietin. In this work, the techniques of semi-blind robust identification is used to derive such models. These models are suitable for feedback controller design methods including robust control design techniques. As such, the robust $\mu / H_{\infty}$ controller was implemented using the derived models to improve on treatment strategy in anemia patients. Additionally, a procedure is provided to implement robust control design technique on newly arrived patients with limited measurement samples to generate individualized models. 


\subsection{DIRECTIONS FOR FUtURE RESEARCH}

There are several possibilities for future work related to this proposal. In this last section we provide few of such possibilities, but certainty not limited to the list:

- Decay rate parameter determination: Currently, heuristic approach is used to select the decay rate parameter for the semi-blind robust identification. This parameter value is an application specific parameter that must be known prior to the identification technique. A means of deriving this parameter value from measurement samples alone will greatly speed up the identification process.

- Automate the identification process with a user friendly interface: The current identification and control procedures rely on sequence of codes that must be executed. Using these sets of codes may pose a challenge to certain end-users, particularly those in clinical fields who may be unfamiliar with the source codes. A solution to this problem is to design a user-friendly interface to provide easeof-use for these individuals.

- Extend the algorithm to incorporate patients with multiple conditions and multiple dosage treatments (MIMO): This work explored treatment of anemia conditions with dosage erythropoietin. An extension to this work might include treatment with multiple health conditions and with multiple dosage medications (MIMO). For instance, patient with kidney condition might also have heart problems that might be administered EPO and Warferin for treatment of the two conditions, respectively. 


\section{REFERENCES}

[1] D. M. Spiegel. Anemia Management in Chronic Kidney Disease : What Have We Learned after 17 Years ? Seminars in Dialysis, 19(4):269-72, 2006.

[2] J. Adamson. The erythropoietin-hematocrit relationship in normal and polycythemic man: implications of marrow regulation. Blood, 32(4):597-609, Oct. 1968.

[3] J. W. Eschbach and J. W. Adamson. Anemia of end-stage renal disease (ESRD). Kidney International, 28(1):1-5, Jul. 1985.

[4] F. Falderrabano. Erythropoietin in chronic renal failure. Kidney International, 50(4):1373-91, Oct. 1996.

[5] E. F. Unger, A. M. Thompson, M. J. Blank, and R. Temple. Erythropoiesisstimulating agents-time for a reevaluation. New England Journal of Medicine, 362(3):189-192, 2010. PMID: 20054037.

[6] NKF-QOQI. Clinical practice guidelines and clinical practice recommendations for anemia in chronic kidney disease. Am J Kidney Dis, 30(4):S192-S240, Oct. 1997.

[7] S. Vozeh and J.-L. Steimer. Feedback Control Methods for Drug Dosing Optimization: Concept, Classification, and Clinical Application. Clinical Pharmacokinetics, 10:457-476, 1985.

[8] D. Linkens and S. S. Hacisalihzade. Computer control systems and pharmacological drug administration: a survey. Journal of Medical Mngineering \& Technology, 14(2):41-54, 1990.

[9] S. S. Hacisalihzade. Control engineering and therapeutic drug delivery. IEEE Control Systems Magazine, pages 44-45, Jun. 1989.

[10] J. Borowiec and A. Tzes. Application of supervisory impulse response interpolation scheme for system identification of flexible structures. In American Control Conference, Proceedings of the 1995, volume 2, pages 1423-1427 vol.2, 1995.

[11] H. Kang, Y. Qi, G. Liu, J. Liu, and C. Li. Application of the arma model in non-stationary vibration signals. In Quality, Reliability, Risk, Maintenance, and Safety Engineering (ICQR2MSE), 2012 International Conference on, pages 751754, 2012. 
[12] A. Dahlen. Identification of stochastic systems: Subspace methods and covariance extention. Phd, KungL Tekniska Hogskolan, 2001.

[13] P. M. Makila and J. Partington. Robust identification of strongly stabilizable systems. IEEE Transactions on Automatic Control, 37(11):1709-1716, 1992.

[14] A. Helmicki, C. Jacobson, and C. Nett. Fundamentals of control-oriented system identification and their application for identification in H. In American Control Conference, 1991, pages 89-99. IEEE, 1991.

[15] A. Helmicki, C. Jacobson, and C. Nett. Control oriented system identification: a worst-case/deterministic approach in $H_{\infty}$. IEEE Transactions on Automatic Control, 36(10):1163 -1176, Oct. 1991.

[16] A. Helmicki, C. Jacobson, and C. Nett. Identification in $H_{\infty}$ : Linear algorithm. IEEE Transactions on Automatic Control, 36:1163 -1176, Oct. 1991.

[17] R. S. S. Pena and C. Galarza. Practical issues in robust identification. IEEE Transactions on Control Systems Technology, 2(1):54-56, 1994.

[18] M. C. Mazzaro, P. A. Parrilo, and R. S. S. Pena. Robust identification:an approach to select the class of candidate models. International Journal of Control, 74(12):1210-1218, 2001.

[19] T. Inanc. Mixed $\ell_{1} / H_{\infty}$ robust identification: Application to a flexible structure testbed. Master's thesis, Pennsylvania State University, 1996.

[20] J. R. Partington. Robust Identification in $H_{\infty}$. Journal of Mathematical Analysis and Applications, 166:428-441, 1992.

[21] A. Helmicki, C. Jacobson, and C. Nett. Worst-case/deterministic identification in $H_{\infty}$ : the continuous-time case. IEEE Transactions on Automatic Control, 37(5):604-610, 1992.

[22] J. Chen, C.Nett, and M.Fan. Worst-case system identification in $H_{\infty}$ : Validatation of a priori information, essentially optimal algorithms and error bounds. In Proc. Ameri. Control Conference, 36:1163 -1176, Oct. 1991.

[23] G. Gu and P. Khargonekar. A class of algorithms for identification in hinfinity. In Decision and Control, 1991., Proceedings of the 30th IEEE Conference on, pages 634-639 vol.1, 1991.

[24] C. J. C. N. J. Partington. Worst case system identification in 11: Optimal algorithms and error bounds. Systems \& Control Letters, 19:419-424, 1992.

[25] C. N. Nett. The caratheory-fejer problem and $H_{\infty}$ identification: a time domain approach. IEEE Transaction on Decision and control, pages 68-73, 1993. 
[26] T. Theodosopoulos. Worst-case identification in $\ell_{1}$ : algorithms and complexity. Systems, 22(2):93-98, Feb. 1993.

[27] J. Chen, C.Nett, and M.Fan. Optimal nonparametric system identification from arbitrary corrupt finite time series: A control oriented approach. In Proc. Ameri. Control Conference, pages 279-285, 1992.

[28] B. Kacewicz and M. Milanese. Optimal finite-sample experiment design in the worst-case $\ell_{1}$ system identification. IEEE In proceding of Decision and Control, pages 56-61, 1992.

[29] A. Khosravi and A. Jalali. Low-order 11 optimal control design via lmi. In IEEE International Symposium on Intelligent Signal Processing, pages 1-5, 2007.

[30] P. A. Parrilo, R. S. S. Pena, and M. Sznaier. A parametric extension of mixed time/frequency robust identification. IEEE Transactions on Automatic Control, 44(2):364-369, 1999.

[31] H. Akçay and B. Ninness. Rational Basis Functions for Robust Identification from Frequency and Time-Domain Measurements. Automatica, 34(9):1101-1117, Sep. 1998.

[32] J. Schoukens, Y. Rolain, and R. Pintelon. On the use of parametric and nonparametric noise-models in time- and frequency domain system identification. In 49th IEEE Conference on Decision and Control (CDC), pages 316 -321, Dec. 2010.

[33] T. Inanc, M. Sznaier, P. Parrilo, and R. Pena. Robust identification with mixed parametric/nonparametric models and time/frequency-domain experiments: theory and an application. IEEE Transactions on Control Systems Technology, 9(4):608-617, Jul. 2001.

[34] W. Ma. Semi-Blind Robust Identification and Model (in)validation. PhD thesis, The Pennsylvania State University, Dec. 2007.

[35] J. Chen and G. Gu. Control Oriented System Identification: An $H_{\infty}$ Approach. John Wiley \& Sons, Inc., New York, first edition, Jun. 2000.

[36] R. Smith and J. Doyle. Model validation: a connection between robust control and identification. IEEE Transactions on Automatic Control, 37(7):942-952, Jul. 1992.

[37] M. M. Livstone, M. Dahleh, and J. Farrell. A framework for robust control based model invalidation. In American Control Conference, 1994, volume 3, pages 3017-3020 vol.3, 1994.

[38] S. Thapliyal, Y. Faitakis, and J. Kantor. A model validation approach to fault detection. In American Control Conference, 1994, volume 3, pages 3027-3028 vol.3, 1994. 
[39] W. Liu and J. Chen. Model validation in 11 using frequency-domain data. In IEEE Conference on Decision and Control, volume 6, pages 6509-6514 Vol.6, 2003.

[40] O. Toker and J. Chen. On computational complexity of invalidating structured uncertainty models. Systems \& Control Letters, 33(3):199-207, 1998.

[41] S. Boyd, L. E. Ghaoui, E. Feron, and V. Balakrishnan. Linear Matrix Inequalities and Control Theory. Society from Industrial and Applied Mathematics, 1994.

[42] A. C. Guyton and J. E. Hall. Textbook of Medical Physiology. Elsevier Sanders, 11 edition, 2005.

[43] A. Gaweda. Improving management of anemia in end stage renal disease using reinforcement learning. International Joint Conference on Neural Networks, pages 953-958, 2009.

[44] S. Fishbane and J. S. Berns. Hemoglobin cycling in hemodialysis patients treated with recombinant human erythropoietin. Kidney International, 68:1337-1343, 2005.

[45] R. Bellazzi, C. Siviero, and R. Bellazi. Mathematical modeling of erythropoietin therapy in uremic anemia. does it improve cost-effectiveness? Medical decision making and problem solving in hematology, 79:154-164, 1994.

[46] R. Bellazzi. Drug Delivery Optimization through Bayesian Networks: An Application to Erythropoietin Therapy in Uremic Anemia. Computers and Biomedical Research, 26(3):274-293, Jun. 1993.

[47] M. K. Muezzinoglu. Approximate Dynamic Programming for Anemia management. $\mathrm{PhD}$ thesis, University of Louisville, Mar. 2006.

[48] D. E. Uehlinger, F. A. Gotch, and L. B. Sheiner. A pharmacodynamic model of erythropoietin therapy for uremic anemia. Clinical Phamacology and Therapeutics, 51:76-89, 1992.

[49] D. Westwick and R. Kearney. Identification of physiological systems: a robust method for non-parametric impulse response estimation. Medical \& Biological Engineering \& Computing, 35(2):83-90, Mar. 1997.

[50] R. Bellazi. Drug delivery optimization through bayesian networks. AMIA, pages 572-578, 1993.

[51] K. G. Gadkar, R. Gunawan, and F. J. D. III. Iterative approach to model identification of biological networks. BMC Bioinformatics, 6, Jun. 2005.

[52] B. J. M. Bailey and W. M. Haddad. Drug Dosing Control in clinical pharmacology. IEEE Control Systems Magazine, (April):35-51, 2005. 
[53] A. E. Gaweda, A. Jacobs, M. E. Brier, and J. M. Zurada. Pharmacodynamic population analysis in chronic renal failure using artificial neural networks-a comparative study. Neural networks : the official journal of the International Neural Network Society, 16(5-6):841-5, 2003.

[54] A. Gaweda, A. Jacobs, and M. Brier. Application of fuzzy logic to predicting erythropoietic response in hemodialysis patients. The International Journal of Artificial Organs, 31(12):1035, 2008.

[55] A. E. Gaweda, M. K. Muezzinoglu, G. R. Aronoff, A. A. Jacobs, J. M. Zurada, and M. E. Brier. Individualization of pharmacological anemia management using reinforcement learning. Neural Networks, 18:826-834, 2005.

[56] J. D. Martin-Guerrero, E. S. Olivas, G. C. Valls, A. J. S. Lopez, J. J. P. Ruixo, and N. J. Torres. Use of neural networks for dosage individualisation of erythropoietin in patients with secondary anemia to chronic renal failure. Computers in Biology and Medicine, 33(4):361-373, Jul. 2003.

[57] R. Bellazzi, C. Siviero, and R. Bellazzi. Mathematical modeling of erythropoietin therapy in uremic anemia. does it improve cost-effectiveness? Hemoglobin, pages 154-164, 1994.

[58] J. D. Martin-Guerrero, G. Camps-Valls, E. Soria-Olivas, A. J. Serrano-Lopez, J. J. Perez-Ruixo, and N. V. Jimenez-Torres. Dosage individualization of erythropoietin using a profile-dependent support vector regression. IEEE Transactions on Biomedical Engineering, 2003.

[59] L. Ljung. System identification: theory for the user. Prentice Hall PTR, Upper Saddle River, NJ, USA, 2nd edition, 1999.

[60] D. Ludvig and E. Perreault. System identification of physiological systems using short data segments. IEEE Transactions on Biomedical Engineering, 59(12):35413549, 2012.

[61] M. Casini. Set-Membership Estimation: An Advanced Tool for System Identification. PhD thesis, Univesita Degli Studi Di Siena, 2002.

[62] M. Milanese and A. Vicino. Optimal estimation theory for dynamic systems with set membership uncertainty: An overview. Automatica, 27(6):997 - 1009, 1991.

[63] M. Milanese and R. Tempo. Optimal algorithms theory for robust estimation and prediction. IEEE Transactions on Automatic Control, 30(8):730-738, 1985.

[64] B. Ninness and G. Goodwin. Estimation of model quality. Automatica, 31:32-74, 1994.

[65] A. P. Sage and J. L. Melsa. System Identification. Academic Press, New York, 1971. 
[66] T. C. Hsiz. System Identification: Least-Squares Methods. Lexington Books, Lexington, Mass., 1977.

[67] L. Ljung. Convergence analysis of parametric identification methods. IEEE Transactions on Automatic Control, 23(5):770-783, 1978.

[68] Y. Chen. Lecture Notes : Week 3a ECE / MAE 7360 Optimal and Robust Control, 2003.

[69] W. Chen, S. Chang, and W. Zhang. Linear matrix inequality-based repetitive controller design for linear systems with time-varying input delay. IET Control Theory \& Applications, 4(6):1071, 2010.

[70] A. Onatski and N. Williams. Modeling model uncertainty. International Seminar on Macroeconomics (A Working Paper), Aug. 2002.

[71] P. M. V. den Hof and X. Bombois. Identification for control. Control, Mar. 2004.

[72] E. Huselstein and H. Garnier. An approach to continuous-time model identification from non-uniformly sampled data. In IEEE Conference on Decision and Control, volume 1, pages 622-623. IEEE, Dec. 2001.

[73] G. Gu and J. Chen. A Nearly Interpolatory Algorithm for $H_{\infty}$ Identification with Mixed Time and Frequency Response Data, Jun. 2000.

[74] P. Parrilo, M. Sznaier, R. S. Pena, and T. Inanc. Mixed time/frequency-domain based robust identification. Automatica, 34(11):1375 - 1389, 1998.

[75] W. Ma, M. Yilmaz, M.Sznaier, and C. Lagoa. Semi-blind robust identification model (in)validation with application to macro-economic modeling. IFAC, 2005.

[76] M. Yilmaz. Robust Systems Theory Applications To Macroeconomic. PhD thesis, The Pennsylvania State University, Dec. 2005.

[77] W. Ma. Semi-Blind Robust Identification and Model (in)validation. PhD thesis, The Pennsylvania State University, Dec. 2007.

[78] O. Camps, H. Lim, C. Mazzaro, and M. Sznaier. A caratheodory-fejer approach to robust multiframe tracking. In Computer Vision, 2003. Proceedings. Ninth IEEE International Conference on, pages 1048-1055 vol.2, Oct. 2003.

[79] R. Bellazzi, C. Siviero, and R. Bellazzi. Mathematical modeling of erythropoietin therapy in ure- mic anemia. does it improve cost-effectiveness? Hemoglobin, pages 154-164, 1994.

[80] H. Huang, E. Haber, L. Horesh, and J. K. Seo. Optimal estimation of $\ell_{1}$ regularization prior from a regularized empirical bayesian risk standpoint. Inverse Problems and Imaging, 6:447-464, 2012. 
[81] D. Miskulin, D. Weiner, H. Tighiouart, V. Ladik, K. Servilla, P. Zager, A. Martin, H. Johnson, and K. Meyer. Computerized decision support for EPO dosing in hemodialysis patients. American Journal of Kidney Diseases, 54(6):1081-1088, 2009.

[82] A. E. Gaweda, B. H. Nathanson, A. A. Jabos, M. G. George Aronoff, and M. Brier. Determining Optimum Hemoglobin Sampling for Anemia Management from Every-Treatment Data. American Society of Nephrology, 5:1939-1945, 2010.

[83] S. Skogestad and I. Postlethwaite. Multivariable Feedback Control: Analysis and Design. John Wiley \& Sons, 2005.

[84] J. Bird. Engineering Mathematics. Elsevier, 2007. 


\title{
CURRICULUM VITAE
}

\author{
Augustine E. Akabua
}

\begin{abstract}
ADDRESS
Electrical and Computer Engineering Department

University of Louisville

Louisville, KY. 40292
\end{abstract}

\section{EDUCATION}

May 2006 B.S., Electrical Engineering, University of Louisville

Louisville, KY. 40292

May 2007 M.S., Electrical Engineering, University of Louisville

Louisville, KY. 40292

August 2014 Ph.D., Electrical Engineering, University of Louisville

Louisville, KY. 40292

\section{AWARDS}

Spring 2014 Doctoral Dissertation Completion Award, University of Louisville. Louisville, KY. 


\section{PublicATIONS}

Akabua, E., Inanc T., Gaweda A., Brier M.E., Kim S., Zurada M.J. "Robust Identification Approach to Individualized Anemia Model." Journal of Applied Computer Science Methods, 3(2):65-75, 2011.

Akabua, E., Inanc T., Gaweda A., Brier M.E., Kim S., Zurada M.J. "Individualized Model Discovery: The Case of Anemia Patients" 2013. Submitted to Computer Methods and Programs in Biomedicine.

Akabua, E., and Inanc T. "Preliminary Results: Robust versus Classical Identification Approaches to Individualized Anemia Patient Modeling." $36^{\text {th }}$ Annual International IEEE EMBS Conference. Chicago,IL.

Akabua, E. and Inanc T. "Semi-Blind Robust Identification Approach to Erythropoiesis Modeling in End-Stage Renal Disease." Submitted to IEEE Engineering in Medicine and Biology Society 